\title{
Duality covariant multi-centre black hole systems
}

\author{
Guillaume Bossard $^{a}$ and Stefanos Katmadas ${ }^{a, b}$ \\ ${ }^{a}$ Centre de Physique Théorique, École Polytechnique, CNRS, \\ 91128 Palaiseau, France \\ ${ }^{b}$ Institut de Physique Théorique, CEA Saclay, CNRS-URA 2306, \\ 91191 Gif sur Yvette, France \\ E-mail: guillaume.bossard@cpht.polytechnique.fr, \\ stefanos.katmadas@cpht . polytechnique.fr
}

ABSTRACT: We present a manifestly duality covariant formulation of the composite nonBPS and almost-BPS systems of multi-centre black hole solutions in four dimensions. The method of nilpotent orbits is used to define the two systems in terms of first order flow equations that transform covariantly under the duality group. Subsequently, we rewrite both systems of equations in terms of real, manifestly duality covariant, linear systems of Poisson equations. Somewhat unexpectedly, we find that the two systems are naturally described by the same equations involving space dependent abelian isometries that are conjugate to T-dualities by similarity transformations.

KEYwORDS: Black Holes, Supergravity Models

ARXIV EPRINT: 1304.6582 


\section{Contents}

1 Introduction and overview 1

1.1 Overview of results 4

$\begin{array}{llr}2 & \text { T-dualities } & \mathbf{7}\end{array}$

$\begin{array}{lll}2.1 & \text { Symmetric special Kähler spaces } & 10\end{array}$

2.2 Freudenthal ternary algebra realisation of $G_{4} \quad 12$

$\begin{array}{lll}2.3 & \text { T-dualities in the complex basis } & 16\end{array}$

3 The $c^{*}$-map, nilpotent orbits and first order systems $\quad 20$

3.1 The three-dimensional non-linear sigma model 21

$\begin{array}{lll}3.2 & \text { Nilpotent orbits } & 23\end{array}$

$\begin{array}{lll}3.3 & \text { Composite non-BPS flows } & 25\end{array}$

$\begin{array}{lll}3.4 & \text { Almost-BPS flows } & 27\end{array}$

4 Composite non-BPS system $\quad 29$

$\begin{array}{lll}4.1 & \text { The electromagnetic potentials } & 30\end{array}$

$\begin{array}{lll}4.2 & \text { Connection to T-dualities } & 31\end{array}$

4.3 The linear system 33

4.4 Integration and local structure 34

$\begin{array}{lll}4.5 & \text { Summary of results } & 37\end{array}$

5 Almost-BPS system $\quad 40$

5.1 The electromagnetic potentials 41

5.2 Connection to T-dualities 43

$\begin{array}{lll}5.3 & \text { The linear system } & 44\end{array}$

$\begin{array}{lll}5.4 & \text { Integration and local structure } & 45\end{array}$

5.5 Summary of results 48

6 Conclusion $\quad 50$

A $\mathcal{N}=2$ supergravity and symmetric special Kähler geometry 52

\section{Introduction and overview}

The structure of black hole solutions in the supergravity effective description of string/Mtheory compactifications has long been a useful tool in understanding their microscopic realisation in string theory. In the supersymmetric (BPS) case, the supergravity black hole solutions [1-3] have been understood microscopically to be described by D-branes wrapping supersymmetric cycles $[4,5]$. Nonetheless, all microscopic BPS configurations cannot 
correspond to single centre solutions in the effective $\mathcal{N}=2$ supergravity description, and it has been understood [6] that the latter then describe composite bound states of BPS black holes [6-9]. The detailed description of these solutions and their domain of stability in moduli space was very important in order to correctly reproduce the corresponding microscopic results $[10,11]$.

In the aim of generalising this understanding to more realistic non-supersymmetric black holes, the first non-trivial step is to consider non-supersymmetric extremal black holes. One can classify extremal black hole solutions into two main classes: the overrotating class, for which the angular momentum saturates the extremality bound, and the under-rotating class, for which the electro-magnetic charges saturate the extremality bound, and which includes the supersymmetric solutions. Given that a stationary spacetime defines a time fibration over a space-like three-dimensional base, one may distinguish the various classes by their corresponding base space. The characteristic of all known under-rotating extremal solutions is that the three-dimensional base space is flat, i.e. $\mathbb{R}^{3}$ with the Euclidean metric, whereas the over-rotating extremal solutions all admit the singular three-dimensional base of the extremal Kerr solution. In this paper we will only discuss under-rotating solutions admitting a flat three-dimensional base space. ${ }^{1}$

Within the class of under-rotating solutions with an $\mathbb{R}^{3}$ base, there are two known interacting systems of non-BPS black holes. The composite non-BPS system [12-14] describes the interactions of black holes that are non-BPS in isolation, while the almost-BPS system [15-17], allows for configurations of centres that are be both BPS and non-BPS in isolation. Both these systems have been studied in some detail and, in several cases, the known explicit solutions are general enough to allow for the most general solution to be obtained by dualities (i.e. symmetries of the three-dimensional theory).

An important feature of the existing formulations of both these non-BPS systems is the existence of distinguished directions in charge space, such that some are associated to harmonic functions as in the BPS system, whereas others are associated to functions solving Poisson equations. The different directions being then intrinsically inequivalent, one cannot straightforwardly rotate one into another by duality, as for BPS solutions. It is therefore customary to solve these equations and construct solutions parametrised by integration constants that can only be related to charges and asymptotic moduli a posteriori. Of course, one may straightforwardly apply a duality rotation on the solutions to change the identification of pre-determined charges, thus covering all possible charge configurations. This process is however not only cumbersome, but more importantly it obscures the overall structure of these systems, which should be manifest in a fully covariant formulation.

A further motivation for generalising the existing formulations is that non-supersymmetric under-rotating extremal black holes seem to admit a microscopic description similar to their BPS cousins $[18,19]$. Understanding the domains of existence and stability of these solutions will eventually be important in order to understand in more detail their microscopic description. However, despite the existence of explicit solutions describing bound

\footnotetext{
${ }^{1}$ In the five dimensional uplift these solutions feature more elaborate base spaces, which however still include a flat $\mathbb{R}^{3}$.
} 
states of such non-BPS black holes, their domain of existence in moduli space has not been studied in detail. One of the main obstacles in carrying out this program originates from the property that these solutions are described in terms of parameters that are not the physical charges themselves, as explained above. In order to solve this problem, it is important to obtain the general solutions associated to fixed charge configurations. Being able to do this in a straightforward manner requires the definition of covariant equations that are not constrained to a fixed duality frame.

In this paper, we provide a manifestly duality covariant formulation of both the composite non-BPS and the almost-BPS systems that unifies their description, by rewriting these systems of linear differential equations in terms of duality covariant quantities, similar to the BPS system [6]. Although these systems of equations turn out to be more complicated than the BPS one, the formalism permits to compute the most general solutions associated to fixed charge configurations. The construction of explicit solutions within these systems will be discussed in a forthcoming publication.

Somewhat surprisingly, we find that both systems are naturally formulated by considering space dependent translations along abelian isometries of the scalar manifold. As all equations are written in terms of objects transforming linearly under electromagnetic dualities, it is natural to introduce local generators for the abelian subgroups corresponding to these isometries. The relevant equations in both the composite non-BPS and the almostBPS can then be expressed in terms of a covariant derivative that contains a nontrivial connection in the Lie algebra of the relevant abelian isometries.

In a specific frame, these abelian isometries coincide with the isometries that generalise the action of T-dualities combined with large gauge transformations of the $p$-form gauge fields present in string theory, for continuous parameters. In general, they will define abelian subgroups that are homomorphic to the latter by similarity transformations. For simplicity, we will refer to them as T-dualities in this paper, despite the fact that they are not in general associated to any duality in string theory. The set of all abelian isometries in the scalar target space of symmetric models has been considered before in the context of (non-)BPS black holes in different guises, see e.g. [20, 21].

The paper is organised as follows. The remainder of this introductory section is devoted to an informal presentation of the method we use to obtain the duality covariant formulation of the composite non-BPS and almost-BPS systems. In doing so, we also explain in more detail way how the T-dualities arise in both systems and in fact allow us to define their action on the relevant quantities without introducing explicit matrix representations. In section 2 we present the general action of arbitrary T-dualities in terms of symplectic vectors and discuss the corresponding decomposition of the charge vector space. In section 3 we consider the three dimensional Euclidean coset non-linear sigma model describing stationary black hole solutions. We then explicitly solve the nilpotency conditions on the scalar momentum as a Lie algebra element for both the composite non-BPS and the almost-BPS system, to obtain the first order flow equations describing each class. We then go on in sections 4 and 5 to rewrite these flow equations as a linear system of Poisson equations for a set of symplectic vectors parametrising the solutions in an arbitrary duality frame. The reader interested in applications can find summaries of the two systems in 
sections 4.5 and 5.5, which are self contained and only require the definition of T-dualities in section 2.2. We conclude in section 6 , where we discuss some of the implications of the local T-dualities acting on both systems and comment on further generalisations.

\subsection{Overview of results}

In order to find systems describing stationary black hole solutions, we consider the reduction along time to a three-dimensional Euclidean theory. In this setting, one can straightforwardly dualise all vector fields to scalars, to obtain a non-linear sigma model coupled to Euclidean gravity. In this paper, we only consider $\mathcal{N}=2$ supergravity theories with a symmetric scalar space, so that the scalar fields of the resulting non-linear sigma model over a pseudo-Riemannian symmetric space. In the case of solutions with a flat base space, there is a powerful method for obtaining first order flow equations that solve the full equations of motion, starting from the observation that the Einstein equation

$$
\operatorname{Tr}\left(P_{\mu} P_{\nu}\right)=R_{\mu \nu}=0,
$$

can be solved by assuming the scalar momentum, $P$, to be a nilpotent element of the Lie algebra. It appears then that the scalar fields equations of motion reduce to a solvable system of differential equations. Based on standard group theoretical considerations, one shows that the nilpotency of $P$ implies that there exists a second element of the algebra, $\mathbf{h}$, such that an eigenvalue equation of the type

$$
\prod_{i=1}^{n}(\mathbf{h}-i) P=0
$$

holds, where $\mathbf{h} P \equiv[\mathbf{h}, P]$ and $n$ is a positive integer. It then follows that $P$ must be a linear combination of eigenvectors of $\mathbf{h}$ with positive eigenvalues. In section 3 we consider the relevant eigenvalue equations for both the composite non-BPS and almost-BPS systems, giving the precise solutions for both the momentum $P$ and the auxiliary fields $\mathbf{h}$. For the composite non-BPS system, this analysis has been given in [14], but is included here for completeness. The corresponding flow equations for the almost-BPS system have been derived for the STU model in [22] by reduction of the second order equations of motion, while we present the general analysis for any symmetric model.

The presence of the aforementioned auxiliary fields is a central feature of both systems, as will become clear from our treatment. In fact, we find it most convenient to keep them throughout, as variables characterising the solutions, despite the fact that one may in principle solve for them in terms of physical fields. In the systems we shall consider, $\mathbf{h}$ can be parametrised in terms of a distinguished direction in the charge vector space (plus a phase for the almost-BPS system). This distinguished direction is associated to a constant so-called very small vector, or in other words a one-charge vector, in the sense that it can always be brought by dualities to a canonical form where it has only one charge, as a pure $D 6$ charge for example. However, one must keep this very small vector arbitrary in order to be able to consider generic charge configurations.

Treating the system in terms of electromagnetic charges and auxiliary vectors implies that one has to work with real quantities, rather than the complex quantities that appear 
naturally in the Euclidean 3-dimensional non-linear sigma model. It turns out that this is essential in solving the system in terms of local functions, since a linear structure only appears after writing all flow equations in the real basis. As mentioned above, this change of basis, that permits to solve non-linear first order equations in terms a solvable system of linear differential equations, is the main technical result of this paper.

In order to appreciate the importance of the real formulation, the simple example of the analogous situation for the BPS system is instructive. In this case the auxiliary element $\mathbf{h}$ in (1.2) is much simpler, as it is parametrised by a single phase, $e^{i \alpha}$. Taking the static case for simplicity, (1.2) leads to the standard BPS equations

$$
\partial_{r} U=r^{-2} e^{U} \operatorname{Re}\left(e^{-i \alpha} Z(\Gamma)\right), \quad \partial_{r} t^{i}=r^{-2} e^{U} e^{i \alpha} \bar{Z}^{i}(\Gamma) .
$$

Here, $r$ is the distance from the horizon, $e^{U}$ is the single function parametrising the metric, $t^{i}$ are the vector multiplet scalars and $Z(\Gamma), Z_{i}(\Gamma)$ are the central charge of the charge vector $\Gamma$, and its Kähler derivative. In this form, it seems a rather non-trivial task to solve (1.3) explicitly. It is however possible to combine the scalar degrees of freedom in a single vector, $e^{-U} e^{-i \alpha} \mathcal{V}$, where $\mathcal{V}$ is the so called symplectic section, which parametrises the scalars $t^{i}$. The two equations (1.3) can then be written as in [6],

$$
2 \partial_{r} \operatorname{Im}\left(e^{-U} e^{-i \alpha} \mathcal{V}\right)=\frac{\Gamma}{r^{2}},
$$

using standard special geometry identities. It is now trivial to solve the last equation as

$$
2 \operatorname{Im}\left(e^{-U} e^{-i \alpha} \mathcal{V}\right)=-\mathcal{H},
$$

where $\mathcal{H}$ is a vector of harmonic functions, whose poles are identified with the charges $\Gamma$. One still has to solve a set of algebraic equations to obtain the scalars $t^{i}$ and $e^{U}$ in terms of harmonic functions [8], but it is important to stress that (1.5) is equivalent to a solution for the physical scalars.

The analogous computation for the non-BPS case includes the auxiliary vector parametrising the element $\mathbf{h}$ and is therefore considerably more involved. For the restricted case of single centre under-rotating black holes, this was done in detail in [14] and the result was given in terms of the objects appearing in (1.5), together with the vectors in (1.2) above. This class is naturally part of both the multi-centre systems considered in this paper and its description is crucial for understanding the structure of the more general classes. Explicitly, for the single centre class we have [14, 23]

$$
2 \operatorname{Im}\left(e^{-U} e^{-i \alpha} \mathcal{V}\right)=-\mathcal{H}+2 \frac{\left\langle\mathcal{H}, R^{*}\right\rangle}{\left\langle R, R^{*}\right\rangle} R+\frac{M}{\left\langle\mathcal{H}, R^{*}\right\rangle} R^{*},
$$

where $\mathcal{H}$ is again a vector of harmonic functions describing the charges of the black hole, while three new objects appear, namely the function $M$ and the two constant very small symplectic (pseudo-charge) vectors $R$ and $R^{*}$, which are not mutually local, i.e. $\left\langle R, R^{*}\right\rangle \neq 0$.

The presence of two different very small vectors in (1.6) can be somehow surprising, but it is important to note that they do not define independent parameters. Indeed, we 
find that these vectors are determined by the electromagnetic charges $\Gamma$, up to duality transformations leaving $\Gamma$ invariant [14], so that they are determined in terms of $\Gamma$ and the asymptotic scalar fields $t_{\infty}^{i}$. Conversely, one can view the charges, $\Gamma$, and the harmonic vector $\mathcal{H}$ as being constrained to lie in a Lagrangian subspace determined by $R$ and $R^{*}$. The terms proportional to the two very small vectors are worth discussing in more detail. First, note that the term proportional to $R$ is simply a projection on that component of $\mathcal{H}$ with an additional factor of 2 , implying that this particular component appears with a flipped sign. This is a very general feature of non-BPS solutions that has been observed in many examples in the literature [24-26]. On the other hand, the component along $R^{*}$ in (1.6) contains the function, $M$, which represents the only genuinely new term in the expression for the scalars, and is constrained to be a dipole harmonic function characterising the angular momentum of the under-rotating non-BPS black hole [16].

In sections 4 and 5, we explicitly solve the composite non-BPS and almost-BPS nonlinear first order systems derived in section 3 in terms of real vectors of local functions. As we shall see, the generalisation to multi-centre systems can be performed by allowing one of $R$ or $R^{*}$ to vary in space, while keeping their symplectic product fixed $\left\langle R, R^{*}\right\rangle=4$. The space dependence of the non-constant vector can then be reabsorbed into a space dependent duality transformation that leaves the constant very small vector invariant. Because this duality transformation lies in an abelian subgroup that is conjugate to the group of T-dualities by similarity transformations, we shall simply refer to them as T-dualities. In particular, the composite non-BPS system is described by the T-dualities, $\mathrm{T}^{+}$, defined as leaving $R$ invariant, while the almost-BPS system is described by the T-dualities, $\mathrm{T}^{-}$, leaving $R^{*}$ invariant. One then shows that the symplectic section $\mathcal{V}$ still takes the form (1.6), with $\mathcal{H}$ constrained to lie in the same Lagrangian subspace determined by $R$ and $R^{*}$. Since one of the very small vectors is not constant, $\mathcal{H}$ is not harmonic anymore, but satisfies the Poisson equation

$$
d \star d\left(\exp \left[-\mathrm{T}^{ \pm}\right] \mathcal{H}\right)=d \mathrm{~T}^{ \pm} \wedge \star d \mathrm{~T}^{ \pm} \exp \left[-\mathrm{T}^{ \pm}\right] \mathcal{H}, \quad d \star d \mathrm{~T}^{ \pm}=0,
$$

where the second equation imposes that the $n_{v}$ parameters of the T-dualities are given by arbitrary harmonic functions. The function $M$ in (1.6) is also specified by a T-duality covariant equation, given by

$$
\star d \omega-d M=\left\langle\mathcal{H}, d \mathcal{H}-2 d \mathrm{~T}^{ \pm} \mathcal{H}\right\rangle,
$$

which also fixes the angular momentum one-form, $\omega$. Although it is not manifest from these general equations, the graded structure of the vector space is such that the system is solvable. Because the two T-dualities in the right hand side of (1.7) have a nontrivial kernel, it follows that the source in (1.7) does not contain some of the components of $\exp \left[-\mathrm{T}^{ \pm}\right] \mathcal{H}$, which therefore includes both harmonic and non-harmonic components. It turns out that $\mathrm{T}^{ \pm}$act as raising or lowering operators, so that only the harmonic components turn out to source the non-harmonic ones, i.e. the non-harmonic components of $\exp \left[-\mathrm{T}^{ \pm}\right] \mathcal{H}$ do not source themselves. These properties will be discussed in detail in sections 4 and 5 .

By definition, T-dualities are represented in terms of symplectic matrices in the $2\left(n_{v}+\right.$ 1)-dimensional vector space of charges. However, as will be shown in detail in the following 
sections, the $n_{v}$ parameters of a T-duality can be arranged into a symplectic vector obeying a number of constraints. One can therefore write the action of the corresponding T-duality in terms of this vector, together with $R$ and $R^{*}$, using the symplectic product and the quartic symmetric invariant $I_{4}$ (which defines the entropy of extremal static black holes). In this way, we obtain explicit expressions for the sources in (1.7), in terms of $\exp \left[-\mathrm{T}^{ \pm}\right] \mathcal{H}$ and the parameters of the T-dualities, that can be evaluated explicitly once a model and its associated quartic invariant are specified. These equations reduce to the systems introduced in $[12,15]$, for a particular choice for the two very small vectors above.

This concludes our short presentation of the main results in this paper. For the convenience of the reader, we provide an account of the results appearing in the following sections, which can be read independently of each other with the exception of section 2, that is basic to most applications. In section 2 we give a detailed discussion of the general T-dualities. We show that given two very small vectors $R$ and $R^{*}$ that do not mutually commute, one can explicitly define a graded decomposition of the symplectic vector space, on which the $\mathrm{T}$-dualities $\mathrm{T}^{ \pm}$can be respectively defined as raising and lowering operators. The structure of this decomposition is essential for all applications in this paper.

We then go on in section 3 to define the two systems of non-BPS multi-centre black hole solutions in terms of the non-linear sigma model in three dimensions, obtained after dimensional reduction over the time direction. After an overview of the main properties of this three-dimensional Euclidean theory, we discuss in detail the eigenvalue equations (1.2) for the two systems at hand. This results to two sets of first order flow equations that completely describe the composite non-BPS and almost-BPS systems in four dimensions.

Sections 4 and 5 are mirror copies of each other, wherein we present in detail the change of variables that transforms the flow equations of section 3 to two linear systems. The reader can find a concise summary of the two multi-centre systems in the real formulation in sections 4.5 and 5.5 respectively, where we also discuss some general properties of the solutions, deferring a more detailed presentation and explicit examples for a forthcoming publication [27].

\section{T-dualities}

In this section, we provide a detailed discussion of the abelian isometries on the scalar target space of $\mathcal{N}=2$ supergravity, ${ }^{2}$ that leave a given charge vector invariant. As will be shown in later sections of this paper, the precise action of these isometries, in their most general form, is of central importance in the construction of multi-centre non-BPS black holes. We start with an informal discussion of the simplest example of such abelian isometries. Subsequently, we review some properties of symmetric special Kähler spaces with a cubic prepotential in section 2.1. Section 2.2 is devoted to the definition of the abelian isometries we shall refer to as T-dualities and their explicit action in terms of the associated real vectors. In section 2.3 we discuss the realisation of the same isometries in the complex basis defined by the central charge and its Kähler derivatives.

\footnotetext{
${ }^{2}$ We refer to the appendix for a concise review of our conventions and notations.
} 
Throughout this paper, we study extremal multi-centre black hole solutions in $\mathcal{N}=2$ supergravity coupled to $n_{v}$ vector multiplets labeled by an index $i=1, \ldots, n_{v}$, whose scalar fields, $t^{i}$, parametrise a symmetric special Kähler target space, $\mathcal{M}_{4}$. These spaces were classified some time ago [28] and include minimally coupled vector multiplets, which are not of interest in this work, and theories with a cubic prepotential, specified by a completely symmetric tensor $c_{i j k}$ [29] (cf. (A.5)). The target space geometry is governed by a Kähler potential (cf. (A.7)), which manifestly depends only on the imaginary part of the scalars, $t^{i}$. It follows that the real parts of the scalars are coordinates along $n_{v}$ isometries of the scalar manifold, acting as

$$
t^{i} \rightarrow t^{i}+k^{i}
$$

where $k^{i}$ is a vector of $n_{v}$ constant real parameters. These isometries are generic in all cubic models and clearly form an abelian algebra. For theories originating from Calabi-Yau string compactifications, the operation (2.1) can be viewed as large gauge transformations on the higher dimensional tensor gauge fields along internal cycles, combined with T-dualities.

While this description is useful in characterising the symmetries themselves, for reasons that will become clear below, in this work we are interested in the embedding of these isometries in the symplectic group, which acts on the electric and magnetic gauge fields in four dimensions. The convenient variable to use in order to make the action of the isometries in (2.1) transparent is the so called scalar symplectic section, $\mathcal{V}$, which is a somewhat redundant way of repackaging the scalars, as

$$
\mathcal{V}=\left(\begin{array}{c}
X^{I} \\
F_{I}
\end{array}\right), \quad t^{i}=\frac{X^{i}}{X^{0}},
$$

where $F_{I}$ are the derivatives of the prepotential with respect to the $X^{I}$. The index $I=$ $\{0, i\}$, runs over one more entry than $n_{v}$ and enumerates all the gauge fields in the theory, i.e. the vector multiplet gauge fields and the graviphoton. Note that $\mathcal{V}$ changes under Kähler transformations by a phase and is subject to the constraint (A.6), so that it encompasses only $2 n_{v}$ degrees of freedom, identified with the physical scalars $t^{i}$ s. The advantage of this variable is that, unlike the physical scalars, it transforms linearly under electric/magnetic duality transformations, in exactly the same way as the electromagnetic charges.

For instance, the isometries in (2.1) are described by a linear transformation acting on the charges as

$$
\exp \left[\mathrm{T}_{k}\right]\left(\begin{array}{c}
p^{0} \\
p^{i} \\
q_{i} \\
q_{0}
\end{array}\right) \rightarrow\left(\begin{array}{c}
p^{0} \\
p^{i}+k^{i} p^{0} \\
q_{i}+c_{i j k} k^{j} p^{k}+\frac{1}{2} c_{i j k} k^{j} k^{k} p^{0} \\
q_{0}-k^{i} q_{i}-\frac{1}{2} c_{i j k} k^{j} k^{k} p^{i}-\frac{1}{6} c_{i j k} k^{i} k^{j} k^{k} p^{0}
\end{array}\right)
$$

where we defined the abelian generators $\mathrm{T}_{k}$ for later convenience. One can now easily verify that the same operation (2.3) acting on the section in (2.2) leads to (2.1) for the physical scalars. In this formulation, the connection of the isometries (2.1) to higher dimensional gauge transformations is more transparent, since it has a natural action on the electromagnetic gauge fields. Moreover, this particular set of abelian transformations also arises in the form of spectral flows in conformal field theories describing black holes microscopically. 
The crucial feature of the symplectic embedding of the abelian isometries is, however, that one may generate an infinite number of inequivalent sets of abelian isometries by conjugating the matrix $\exp \left[\mathrm{T}_{k}\right]$ in $(2.3)$ by a general $U$-duality transformation, as in $[21] .^{3}$ These sets of isometries are more complicated than the one in (2.1) and do not commute with it. From a higher dimensional point of view, some of these more general isometries can also be viewed either as large gauge transformations conjugated with generic $T$ - and/or $U$-dualities, or as (generalised) spectral flows in a dual conformal field theory. Here, we refer to them simply as T-dualities for brevity and we focus on the case of symmetric scalar manifolds, which allows for the most general transformations to be described explicitly.

The representation of a generic T-duality in terms of matrices is of course a rather tedious task, which can be circumvented in a natural way, intrinsically tied to the systems of non-BPS black holes we consider. The crucial observation is that there is always a graded decomposition of the vector space in four components, generalising the clear distinction between the various components in (2.3), based on their transformation rule under T-dualities. Indeed, general T-dualities act consistently on each component of the charge space with a fixed homogeneity in the parameters $k^{i}$, which can never exceed three. In particular, there is a distinguished direction that is invariant under the action of any given T-duality, as for example the electric charge $q_{0}$ is left invariant in (2.3). The $q_{0}$ charge is rather special, since it is an example of a so-called very small vector. We will recall the precise definition of such a vector in what follows, but loosely speaking a very small vector can be defined as a 'one charge vector', in the sense that it is $U$-dual to a pure $q_{0}$ charge. Clearly, the distinguished direction that is left invariant under a generic T-duality must then always be a very small vector, given that all such transformations are $U$-dual to the above example.

The relevance of very small vectors for extremal non-BPS solutions arises already in the single centre class $[14,23,30]$, which is naturally described in terms of an auxiliary pair of mutually nonlocal constant very small vectors, constrained by the physical charge vector. As we will show explicitly in sections 4 and 5 below, the corresponding multi-centre systems are naturally described by promoting one of these very small vectors to be not constant. In order to see the connection to T-dualities, consider the very small vector $\hat{R}$ defined such that its only non-vanishing component is $q_{0}=4$. A general very small vector can be parametrised as

$$
S=c\left(1, s^{i} ; \frac{1}{2} c_{i j k} s^{j} s^{k},-\frac{1}{6} c_{i j k} s^{i} s^{j} s^{k}\right)^{T},
$$

where $c$ and $s^{i}$ are allowed to take singular values as long as the components of $S$ are well defined in the limit. ${ }^{4}$ It follows that a general very small vector $\hat{R}^{*}$ satisfying $\left\langle\hat{R}, \hat{R}^{*}\right\rangle=4$ can be parametrised as

$$
\hat{R}^{*}=\exp \left[\mathrm{T}_{s}^{+}\right] R_{0}^{*}=\left(1, s^{i} ; \frac{1}{2} c_{i j k} s^{j} s^{k},-\frac{1}{6} c_{i j k} s^{i} s^{j} s^{k}\right)^{T}
$$

\footnotetext{
${ }^{3}$ Following a common misuse of language, we call $U$-duality transformations all the continuous isometries of the scalar field symmetric space, independently of whether they define or not actual string theory dualities for appropriate integral coefficients.

${ }^{4}$ For example one recovers $\hat{R}$ in the limit $c \rightarrow 0, s^{i} \rightarrow \infty$ with $-c \frac{1}{6} c_{i j k} s^{i} s^{j} s^{k}=4$.
} 
where the only non-vanishing component of $R_{0}^{*}$ is $p^{0}=1$. In the case of a constant vector $\hat{R}^{*},(2.5)$ is simply a convenient parametrisation, but in the more general case when $\hat{R}^{*}$ is not constant, one can assume the parameters $s^{i}$ to be functions of space, to obtain

$$
d \hat{R}^{*}=d \mathrm{~T}_{s}^{+} \hat{R}^{*}=\mathrm{T}_{d s}^{+} \hat{R}^{*},
$$

where we used the abelian property of (2.3). As we will show explicitly in later sections, the composite non-BPS system is naturally characterised by two very small vectors, one constant $\hat{R}$ and one non-constant $\hat{R}^{*}$, which have a non-vanishing constant symplectic product $\left\langle\hat{R}, \hat{R}^{*}\right\rangle=4$. The almost-BPS system is similarly described by two very small vectors, only the role of $\hat{R}$ and $\hat{R}^{*}$ are interchanged. The constant very small vector is left invariant by the relevant T-dualities (as for example (2.3)), which are therefore different for each system. The non-constant very small vector can be expressed as in (2.5) for a constant vector $R_{0}^{*}$ in the composite non-BPS system (or respectively $R_{0}$ for the almost-BPS system), and it follows that it satisfies (2.6). In the rest of the paper $\hat{R}$ and $\hat{R}^{*}$ will be generic very small vectors, and the associated T-dualities will define abelian subgroups conjugate to the one described in (2.3).

\subsection{Symmetric special Kähler spaces}

In this paper we consider $\mathcal{N}=2$ supergravity theories defined in [29] for which the special Kähler target space, $\mathcal{M}_{4}$, is a symmetric space and that can be obtained as Kaluza-Klein reductions of corresponding five dimensional theories. ${ }^{5}$ In this case, $\mathcal{M}_{4}$ is a coset space of the four-dimensional duality group, $G_{4}$, by its maximal compact subgroup $\mathrm{U}(1) \times K_{4}$

$$
\mathcal{M}_{4} \cong\left(\mathrm{U}(1) \times K_{4}\right) \backslash G_{4} .
$$

For the class of theories we consider, the scalar target space is a symmetric space even after dimensional reduction/oxidation to three/five dimensions, so that (2.7) is part of the sequence of embeddings

$$
K_{5} \backslash G_{5} \hookrightarrow\left(\mathrm{U}(1) \times K_{4}\right) \backslash G_{4} \hookrightarrow\left(\mathrm{SL}(2) \times G_{4}\right) \backslash G_{3},
$$

where by $G_{d}, K_{d}$ we denote the duality group and (part of) the isotropy group in $d$ dimensions respectively. Note that the divisor group in three dimensions is non-compact because we consider the time-like reduction to three dimensions, ${ }^{6}$ as that is relevant for the applications we consider later on. Note that $K_{4}$ is the compact real form of $G_{5}$, by property of $\mathcal{N}=2$ supersymmetry.

One can always define a set of vielbeine associated to the Kähler metric $g_{i \bar{\jmath}}$ on $\mathcal{M}_{4}$

$$
g^{i \bar{\jmath}}=e_{a}^{i} e^{a \bar{\jmath}}
$$

such that the constant symmetric tensor

$$
c_{a b c}=i e^{\mathcal{K}} e_{a}^{i} e_{b}^{j} e_{c}^{k} c_{i j k}
$$

\footnotetext{
${ }^{5}$ This excludes theories with minimally coupled vector multiplets, which do not include systems of the type we consider here.

${ }^{6}$ For a spacelike reduction, one obtains $\mathrm{SU}(2) \times K_{3}$, where $K_{3}$ is the compact real form of $G_{4}$.
} 
where $c_{i j k}$ is the $G_{5}$ invariant tensor defining the prepotential (cf. (A.5)), is left invariant by $K_{4}$. Then, the contravariant symmetric tensor $c^{a b c}$ in the conjugate representation satisfies the Jordan identity [29]

$$
c_{f(a b} c_{c d) g} c^{e f g}=\frac{4}{3} \delta_{(a}^{e} c_{b c d)} .
$$

In a complex basis, the Lie algebra of $G_{4}$, denoted $\mathfrak{g}_{4}$ (and respectively $\mathfrak{k}_{4}$ for $K_{4}$ ), naturally decomposes as

$$
\mathfrak{g}_{4} \cong \mathfrak{u}(1) \oplus \mathfrak{k}_{4} \oplus \mathbb{C}^{n_{v}}
$$

It follows that the relevant parameters are given by those corresponding to the elements of $\mathfrak{k}_{4}$, denoted by $G_{b}^{a}$, a real scalar $\gamma$ and a complex vector $\Lambda_{a}$. The corresponding algebra is realised in terms of anticommuting parameters with the nilpotent differential ${ }^{7}$

$$
\begin{aligned}
\delta \Lambda_{a} & =-G^{b}{ }_{a} \Lambda_{b}+2 i \gamma \Lambda_{a} \quad \delta \gamma=\frac{i}{3} \bar{\Lambda}^{a} \Lambda_{a}, \\
\delta G^{a}{ }_{b} & =G^{a}{ }_{c} G^{c}{ }_{b}+c^{a c e} c_{b d e} \Lambda_{c} \bar{\Lambda}^{d}+\bar{\Lambda}^{a} \Lambda_{b}+\frac{1}{3} \bar{\Lambda}^{c} \Lambda_{c} \delta_{b}^{a} .
\end{aligned}
$$

Note that the statement of invariance of the tensor $c_{a b c}$ under $K_{4}$ implies that $\mathcal{N}[\bar{Z}] \equiv$ $\frac{1}{6} c_{a b c} \bar{Z}^{a} \bar{Z}^{b} \bar{Z}^{c}$ is $K_{4}$ invariant for any vector $\bar{Z}^{a}$ transforming in the relevant $n_{v}$-dimensional complex representation of $K_{4}$. One can check that the variation of $G^{a}{ }_{b}$ in (2.13) indeed leaves invariant the cubic norm $\mathcal{N}[Z] \equiv \overline{\mathcal{N}}[\bar{Z}]$ for an anticommuting $\Lambda_{a}$.

The invariance of the cubic norm $\mathcal{N}[Z]$ can be used to define duality invariants and restricted charge vectors, a concept that is of central importance for the applications we consider later in this paper. First, we introduce the quartic invariant for a charge vector $\Gamma$, in terms of its central charges, $Z \equiv Z(\Gamma), Z_{a} \equiv Z_{a}(\Gamma)$, as

$$
I_{4}(\Gamma)=\left(Z \bar{Z}-Z_{a} \bar{Z}^{a}\right)^{2}-c_{e a b} \bar{Z}^{a} \bar{Z}^{b} c^{e c d} Z_{c} Z_{d}+4 \bar{Z} \mathcal{N}[Z]+4 Z \mathcal{N}[\bar{Z}] .
$$

This expression can be verified to be invariant under the $\mathfrak{g}_{4}$ generators of (2.13), so that it is moduli independent. This is manifest by the corresponding real form of this invariant, which is given solely in terms of charges by

$$
\begin{aligned}
I_{4}(\Gamma) & =\frac{1}{4 !} t^{M N P Q} \Gamma_{M} \Gamma_{N} \Gamma_{P} \Gamma_{Q} \\
& =-\left(p^{0} q_{0}+p^{i} q_{i}\right)^{2}+\frac{2}{3} q_{0} c_{i j k} p^{i} p^{j} p^{k}-\frac{2}{3} p^{0} c^{i j k} q_{i} q_{j} q_{k}+c_{i j k} p^{j} p^{k} c^{i l m} q_{l} q_{m},
\end{aligned}
$$

where we also defined the completely symmetric tensor $t^{M N P Q}$ for later reference. Again, one can easily check that (2.15) is invariant under the example T-duality in (2.3), and it is more generally invariant under an arbitrary $G_{4}$ transformation.

We are now in a position to introduce the concept of charge vectors of restricted rank. A generic vector leads to a nonvanishing invariant (2.14)-(2.15) and is also referred to as a rank-four vector, due to the quartic nature of the invariant. Similarly, a rank-three vector, $\Gamma_{3}$, is a vector for which the quartic invariant vanishes, but not its derivative. An obvious

\footnotetext{
${ }^{7}$ The nilpotent differential $\delta$ in these equations acts as $\delta X_{A} T^{A}=\frac{1}{2} X_{A} X_{B}\left[T^{A}, T^{B}\right]$ on a Lie algebra element expressed in a basis of generators $T^{A}$ using a set of anticommuting parameters $X_{A}$.
} 
example is a vector with only $p^{i} \neq 0$ and all other charges vanishing, so that the derivative $I_{4}^{\prime}\left(\Gamma_{3}\right)$ is nonzero and proportional to the cubic term $\mathcal{N}[p]$.

There are two more classes of restricted vectors, defined analogously as rank-two (small) and rank-one (very small) vectors. A rank-two vector, $\Gamma_{2}$, is defined such that both $I_{4}\left(\Gamma_{2}\right)=I_{4}^{\prime}\left(\Gamma_{2}\right)=0$, and a simple example is provided by a vector with all entries vanishing except the $p^{i}$, with the additional constraint that $\mathcal{N}[p]=0$. Finally, a very small vector, $\Gamma_{1}$, is defined such that

$$
\begin{aligned}
I_{4}\left(\Gamma_{1}\right) & =I_{4}^{\prime}\left(\Gamma_{1}\right)=0, \\
\frac{1}{4} I_{4}\left(\Gamma_{1}, \Gamma_{1}, \Gamma, \Gamma\right) & \equiv \frac{1}{4} t^{M N P Q} \Gamma_{1 M} \Gamma_{1 N} \Gamma_{P} \Gamma_{Q}=-\left\langle\Gamma_{1}, \Gamma\right\rangle^{2},
\end{aligned}
$$

for any vector $\Gamma$. In this case, we can also give a general definition in terms of the complex basis, which is in fact independent of the values of the scalar fields. In this paper we will often make use of a rank one vector, $R$, that we choose without loss of generality such that $|Z(R)|=1$. One shows that such a very small vector satisfies

$$
Z(R)=\mathcal{N}[\Omega], \quad Z_{a}(R)=\Omega_{a},
$$

where $\mathcal{N}[\Omega]$ is a phase by construction. The remaining central charges $\Omega_{a}$ are such that

$$
\frac{1}{2} c^{a b c} \Omega_{b} \Omega_{c}=\mathcal{N}[\Omega] \bar{\Omega}_{a}, \quad \bar{\Omega}^{a} \Omega_{a}=3 .
$$

A general very small vector can be obtained by rescaling both $\mathcal{N}[\Omega]$ and $\Omega_{a}$ by a real function. Examples of very small vectors were already given above, as vectors where only the $q_{0}$ or $p^{0}$ component is nonzero, while the parametrisation given in (2.5) is generic up to a possibly singular rescaling.

\subsection{Freudenthal ternary algebra realisation of $G_{4}$}

We now proceed in describing the duality group $G_{4}$, as defined above, in terms of real vector parameters. This is essential for discussing the T-dualities, which are contained in $G_{4}$ as subgroups and can therefore also be described in terms of real vector parameters in the general case, similar to the example (2.3) above.

The central object for the definition of $G_{4}$ in the real basis is the quartic invariant in (2.15) and its derivatives. It is convenient to define a symplectic vector out of the first derivative, $I_{4}^{\prime}(\Gamma)$, of the quartic invariant so that

$$
\left\langle\Gamma, I_{4}^{\prime}(\Gamma)\right\rangle=4 I_{4}(\Gamma), \quad I_{4}^{\prime}(\Gamma, \Gamma, \Gamma)=6 I_{4}^{\prime}(\Gamma) .
$$

Using the definition (2.15) of the quartic invariant and the properties of the rank-three symmetric tensor $c_{i j k}$, one shows the following quintic identity

$$
I_{4}^{\prime}\left(\Gamma, \Gamma, I_{4}^{\prime}(\Gamma)\right)=-8 I_{4}(\Gamma) \Gamma,
$$

for a general charge vector $\Gamma$. This identity is equivalent to the property that the Freudenthal ternary product

$$
(X, Y, Z) \equiv \frac{1}{4} I_{4}^{\prime}(X, Y, Z)+\frac{1}{2}\langle X, Y\rangle Z-\frac{1}{2}\langle Z, X\rangle Y+\frac{1}{2}\langle Y, Z\rangle X,
$$


satisfies the four axioms defined in [31], and inversely, any Freudenthal ternary product is necessarily of the form (2.21), for a completely symmetric rank four tensor satisfying (2.20). One can therefore define the $\mathfrak{g}_{4}$ Lie algebra as in [31]. We shall not use the Freudenthal ternary product, but rather the quintic identity (2.20). We refer to [31] for the more formal definition of the $\mathfrak{g}_{4}$ Lie algebra from the ternary product itself. It is straightforward to combine (2.20) with the symmetry properties of the sextic invariant $\left\langle I^{\prime}\left(\Gamma_{1}\right), I^{\prime}\left(\Gamma_{2}\right)\right\rangle$ to show that for any two vectors $J_{1}$ and $J_{2}$, the linear transformation

$$
\mathbf{g}\left(J_{1}, J_{2}\right) \Gamma \equiv \frac{1}{2} I_{4}^{\prime}\left(J_{1}, J_{2}, \Gamma\right)-J_{1}\left\langle J_{2}, \Gamma\right\rangle-J_{2}\left\langle J_{1}, \Gamma\right\rangle,
$$

preserves both the symplectic product and the quartic invariant. One concludes that (2.22) defines a generator of $\mathfrak{g}_{4}$, and all $\mathfrak{g}_{4}$ generators can in fact be defined in this way. It follows that the Lie algebra takes the form

$$
\left[\mathbf{g}\left(J_{1}, J_{2}\right), \mathbf{g}\left(J_{3}, J_{4}\right)\right]=\mathbf{g}\left(\mathbf{g}\left(J_{1}, J_{2}\right) J_{3}, J_{4}\right)+\mathbf{g}\left(J_{3}, \mathbf{g}\left(J_{1}, J_{2}\right) J_{4}\right),
$$

as shown in [31].

A special case arises for two rank 1 vectors, denoted $R$ and $R^{*}$, which are assumed to be mutually non-commuting. In this case, one can define the corresponding $\mathfrak{g}_{4}$ generator as in $(2.22)$

$$
\mathbf{h}_{T} \Gamma \equiv\left\langle R, R^{*}\right\rangle^{-1}\left(\frac{1}{2} I_{4}^{\prime}\left(R, R^{*}, \Gamma\right)+\left\langle\Gamma, R^{*}\right\rangle R-R^{*}\langle R, \Gamma\rangle\right),
$$

which is central in the description of T-dualities. It is clear from (2.16) that for any rank 1 vector $R$ (or respectively $R^{*}$ ) one has

$$
I_{4}^{\prime}(R, R, \Gamma)=4\langle R, \Gamma\rangle R .
$$

This generator admits therefore $R$ and $R^{*}$ as eigenvectors, with eigenvalues +3 and -3 respectively, and the remaining eigenvectors of $\mathbf{h}_{T}$ can be characterised as follows. Using (2.20) and (2.16), one can show that

$$
\frac{1}{4} I_{4}^{\prime}\left(R, R^{*}, I_{4}^{\prime}\left(R, R^{*}, \Gamma\right)\right)=\left\langle R, R^{*}\right\rangle^{2} \Gamma+3\left\langle R, R^{*}\right\rangle\left(\left\langle\Gamma, R^{*}\right\rangle R+R^{*}\langle R, \Gamma\rangle\right),
$$

(2.26) from which follows the action of the square of $\mathbf{h}_{T}$, as

$$
\mathbf{h}_{T}^{2} \Gamma=\Gamma+8\left\langle R, R^{*}\right\rangle^{-1}\left(\left\langle\Gamma, R^{*}\right\rangle R+R^{*}\langle R, \Gamma\rangle\right) .
$$

This equation implies that $R$ and $R^{*}$ are the unique eigenvectors with eigenvalues +3 and -3 respectively, and also leads to the characteristic equation

$$
\left(\mathbf{h}_{T}^{4}-10 \mathbf{h}_{T}^{2}+9\right) \Gamma=\left(\mathbf{h}_{T}-3\right)\left(\mathbf{h}_{T}-1\right)\left(\mathbf{h}_{T}+1\right)\left(\mathbf{h}_{T}+3\right) \Gamma=0 .
$$

In view of the fact that $\mathbf{h}_{T}$ is symplectic, it follows from (2.27), (2.28) that the $2 n_{v}+2$ electromagnetic charge vector space decomposes into

$$
\mathbb{R}^{2 n_{v}+2} \cong \mathbb{R}^{(-3)} \oplus\left(\mathbb{R}^{n_{v}}\right)^{(-1)} \oplus\left(\mathbb{R}^{n_{v}}\right)^{(1)} \oplus \mathbb{R}^{(3)},
$$


where the two distinguished vectors $R$ and $R^{*}$ are by definition the components of grade 3 and -3 , respectively. This decomposition is clearly relevant to the T-dualities as described in (2.29), as it allows to identify four eigenspaces, based on two very small vectors. The $n_{v}$ eigenvectors of eigenvalue +1 and the $n_{v}$ eigenvectors of eigenvalue -1 can be obtained by defining the corresponding projectors to the four eigenspaces of $\mathbf{h}_{T}$, as $\mathbf{h}_{T} \Gamma^{(n)}=n \Gamma^{(n)}$ for $n=-3,-1,1,3$, i.e.

$$
\begin{aligned}
\Gamma^{(3)} & =\left\langle R, R^{*}\right\rangle^{-1}\left\langle\Gamma, R^{*}\right\rangle R, \\
\Gamma^{(1)} & =\frac{1}{2} \Gamma+\frac{1}{2}\left\langle R, R^{*}\right\rangle^{-1}\left(\frac{1}{2} I_{4}^{\prime}\left(R, R^{*}, \Gamma\right)-3\left\langle\Gamma, R^{*}\right\rangle R+R^{*}\langle R, \Gamma\rangle\right), \\
\Gamma^{(-1)} & =\frac{1}{2} \Gamma-\frac{1}{2}\left\langle R, R^{*}\right\rangle^{-1}\left(\frac{1}{2} I_{4}^{\prime}\left(R, R^{*}, \Gamma\right)-\left\langle\Gamma, R^{*}\right\rangle R+3 R^{*}\langle R, \Gamma\rangle\right), \\
\Gamma^{(-3)} & =\left\langle R, R^{*}\right\rangle^{-1}\langle R, \Gamma\rangle R^{*} .
\end{aligned}
$$

Note that the $\Gamma^{( \pm 1)}$ can simply be identified as the solutions to

$$
\frac{1}{2} I_{4}^{\prime}\left(R, R^{*}, \Gamma^{( \pm 1)}\right)= \pm\left\langle R, R^{*}\right\rangle \Gamma^{( \pm 1)} .
$$

These expressions will be very useful in evaluating the action of T-dualities on general symplectic vectors in the following sections. A further practical advantage of this decomposition is the fact that all inner products must respect the grading, leading to strong constraints on the possible nontrivial combinations. For instance, the grading implies that

$$
I^{\prime}\left(\Gamma^{(-1)}, \Gamma^{(-1)}, R^{*}\right)=0, \quad I^{\prime}\left(\Gamma^{(1)}, \Gamma^{(1)}, R\right)=0,
$$

since there is no vector of weight \pm 5 that these cubic terms could be equal to. Similar considerations apply to scalar products, which necessarily vanish unless the sum of grades of the vectors involved vanishes.

In addition to the decomposition (2.29) of the vector space, the generator $\mathbf{h}_{T}$ implies a corresponding decomposition of the duality group generators. Indeed, $\mathbf{h}_{T}$ commutes with $\mathfrak{g}_{5} \subset \mathfrak{g}_{4}$ and defines the following graded decomposition of $\mathfrak{g}_{4}$

$$
\mathfrak{g}_{4} \cong\left(\mathbb{R}^{n_{v}}\right)^{(-2)} \oplus\left(\mathfrak{g l}_{1} \oplus \mathfrak{g}_{5}\right)^{(0)} \oplus\left(\mathbb{R}^{n_{v}}\right)^{(2)},
$$

where the $\mathfrak{g l}_{1}$ corresponds to $\mathbf{h}_{T}$ itself. Clearly, the $2 n_{v}$ generators of eigenvalue \pm 2 with respect to $\mathbf{h}_{T}$ can be used as raising and lowering operators on the eigenspaces in (2.29). As the reader might already understand, these grade 2 generators are related to the transformations (2.3) by similarity transformations in $G_{4}$.

In terms of the explicit expression (2.22) for the action of $\mathfrak{g}_{4}$, one may consider any grade -1 vector of parameters $k^{(-1)}$ to define the grade 2 generators as

$$
\mathrm{T}_{k}^{+} \Gamma \equiv-\frac{1}{2}\left\langle R, R^{*}\right\rangle^{-1}\left(\frac{1}{2} I_{4}^{\prime}\left(R, k^{(-1)}, \Gamma\right)+\left\langle\Gamma, k^{(-1)}\right\rangle R-k^{(-1)}\langle R, \Gamma\rangle\right) .
$$

This generator is manifestly of grade 2 because of the grading of $R$ and $k^{(-1)}$ themselves and the algebra (2.23). It is convenient to write it in a way that makes the grading explicit

$$
\mathrm{T}_{k}^{+} \Gamma=\left\langle R, R^{*}\right\rangle^{-1}\left(k^{(-1)}\left\langle R, \Gamma^{(-3)}\right\rangle-\frac{1}{4} I_{4}^{\prime}\left(R, k^{(-1)}, \Gamma^{(-1)}\right)-\left\langle\Gamma^{(1)}, k^{(-1)}\right\rangle R\right),
$$


where we used the projections in (2.30) and the fact that $k^{(-1)}$ is of grade (-1). All these generators clearly commute between themselves for different $k^{(-1)}$ 's. Similarly, one defines the grade -2 generator in terms of a grade 1 vector $k^{(1)}$

$$
\begin{aligned}
\mathrm{T}_{k}^{-} \Gamma & \equiv \frac{1}{2}\left\langle R, R^{*}\right\rangle^{-1}\left(\frac{1}{2} I_{4}^{\prime}\left(R^{*}, k^{(1)}, \Gamma\right)-\left\langle k^{(1)}, \Gamma\right\rangle R^{*}+k^{(1)}\left\langle\Gamma, R^{*}\right\rangle\right) \\
& =\left\langle R, R^{*}\right\rangle^{-1}\left(k^{(1)}\left\langle\Gamma^{(3)}, R^{*}\right\rangle+\frac{1}{4} I_{4}^{\prime}\left(R^{*}, k^{(1)}, \Gamma^{(1)}\right)-\left\langle k^{(1)}, \Gamma^{(-1)}\right\rangle R^{*}\right) .
\end{aligned}
$$

The normalisations we have chosen are such that

$$
\mathrm{T}_{k}^{+} R^{*}=k^{(-1)}, \quad \mathrm{T}_{k}^{-} R=k^{(1)},
$$

while one easily computes that

$$
\mathrm{T}_{k}^{+} R=0, \quad \mathrm{~T}_{k}^{-} R^{*}=0 .
$$

In this form, one easily computes that these generators are nilpotent of order 4 , as

$$
\left(\mathrm{T}_{k}^{ \pm}\right)^{4} \Gamma=0
$$

consistent with the grading (2.29), which only allows for four eigenspaces. Explicitly, we find the following expressions for the two sets of generators

$$
\begin{aligned}
&\left(\mathrm{T}_{k}^{+}\right)^{2} \Gamma=-\frac{1}{4}\left\langle R, R^{*}\right\rangle^{-2}\left(I_{4}^{\prime}\left(R, k^{(-1)}, k^{(-1)}\right)\langle R, \Gamma\rangle+I_{4}\left(R, k^{(-1)}, k^{(-1)}, \Gamma\right) R\right), \\
&\left(\mathrm{T}_{k}^{+}\right)^{3} \Gamma=-\frac{1}{4}\left\langle R, R^{*}\right\rangle^{-3} I_{4}\left(R, k^{(-1)}, k^{(-1)}, k^{(-1)}\right)\langle R, \Gamma\rangle R, \\
&\left(\mathrm{~T}_{k}^{-}\right)^{2} \Gamma=\frac{1}{4}\left\langle R, R^{*}\right\rangle^{-2}\left(I_{4}^{\prime}\left(R^{*}, k^{(1)}, k^{(1)}\right)\left\langle\Gamma, R^{*}\right\rangle-I_{4}\left(R^{*}, k^{(1)}, k^{(1)}, \Gamma\right) R^{*}\right), \\
&\left(\mathrm{T}_{k}^{-}\right)^{3} \Gamma=-\frac{1}{4}\left\langle R, R^{*}\right\rangle^{-3} I_{4}\left(R^{*}, k^{(1)}, k^{(1)}, k^{(1)}\right)\left\langle\Gamma, R^{*}\right\rangle R^{*},
\end{aligned}
$$

and moreover

$$
\left[\mathbf{h}_{T}, \mathrm{~T}_{k}^{ \pm}\right]= \pm 2 \mathrm{~T}_{k}^{ \pm}
$$

as in (2.33).

Finally, one also computes using (2.23) and the grading that for any grade -1 vector $e$ and grade 1 vector $f$

$$
\left[\mathrm{T}_{e}^{+}, \mathrm{T}_{f}^{-}\right]=\frac{1}{2} \frac{1}{\left\langle R, R^{*}\right\rangle}\left(\mathbf{g}(e, f)+\langle e, f\rangle \mathbf{h}_{T}\right) .
$$

One can straightforwardly check that

$$
\left(\mathbf{g}(e, f)+\frac{1}{3}\langle e, f\rangle \mathbf{h}_{T}\right) R=0, \quad\left(\mathbf{g}(e, f)+\frac{1}{3}\langle e, f\rangle \mathbf{h}_{T}\right) R^{*}=0,
$$

so that the latter transformation lies in the $\mathfrak{g}_{5} \subset \mathfrak{g}_{4}$ subalgebra, consistently with the graded decomposition (2.33). One can indeed check that these transformations preserve 
the cubic norm $I_{4}\left(R, \Gamma^{(-1)}, \Gamma^{(-1)}, \Gamma^{(-1)}\right)$ for an arbitrary grade -1 vector $\Gamma^{(-1)}$. It turns out that the identity (2.20) implies the associated Jordan identity

$$
I_{4}^{\prime}\left(R^{*}, I_{4}^{\prime}\left(R, \Gamma^{(-1)}, \Gamma^{(-1)}\right), I_{4}^{\prime}\left(R, \Gamma^{(-1)}, \Gamma^{(-1)}\right)\right)=\frac{64}{3}\left\langle R, R^{*}\right\rangle I_{4}\left(R, \Gamma^{(-1)}, \Gamma^{(-1)}, \Gamma^{(-1)}\right) \Gamma^{(-1)},
$$

which generalises (2.11). These equations are clearly valid upon replacing $R$ with $R^{*}$ and $\Gamma^{(-1)}$ by a grade $(+1)$ vector $\Gamma^{(1)}$ throughout.

One may now use the above formulae to identify $\mathrm{T}^{ \pm}$with T-dualities explicitly. Indeed, one can easily check that upon identifying $R$ with the very small vector whose only nonvanishing component $q_{0}$ and $R^{*}$ with its magnetic dual along $p^{0}$, the exponentiated transformations

$$
\exp \left[\mathrm{T}_{k}^{+}\right]=1+\mathrm{T}_{k}^{+}+\frac{1}{2}\left(\mathrm{~T}_{k}^{+}\right)^{2}+\frac{1}{6}\left(\mathrm{~T}_{k}^{+}\right)^{3},
$$

are identical to the spectral flow shown in (2.3). The corresponding set of generators $\mathrm{T}^{-}$ then generate the T-dualities one obtains by conjugating (2.3) by an electric/magnetic duality and leave $R^{*}$ invariant. The grade $(-1)$ and $(+1)$ components are then easily seen to be given by the magnetic, $p^{i}$, and electric components, $q_{i}$, respectively.

In the general case, we can identify all possible sets of T-dualities as given by a choice of $R$ or $R^{*}$, as above, as the generators $\mathrm{T}_{k}^{ \pm}$are entirely determined by the rank 1 vector they leave invariant. Indeed, the characteristic feature of these abelian subgroups is that there is always a unique (up to rescaling) very small vector (e.g. $R$ ) that they leave invariant, whereas they act transitively (up to a rescaling) on the set of very small vectors (e.g. $R^{*}$ ) that are not mutually commuting with the former. In the specific example of (2.3), any very small vector that is not mutually local with $R$ (along $q_{0}$ ) can be obtained by acting with a finite transformation $\exp \left(\mathrm{T}_{k}^{+}\right)$on $R^{*}$ (along $\left.p^{0}\right)$.

In the following sections, we will consider the action of general T-dualities, as we find it convenient to describe multi-centre black hole solutions in terms of two auxiliary very small vectors $R$ and $R^{*}$ that arise naturally from the equations of motion, as mentioned below (2.6). Therefore, we will always consider a T-duality as given explicitly by an exponential as in (2.48), where the explicit action of each order is given by (2.35)-(2.43) above, rather than the equivalent matrix similar to the one in (2.3) that has to be defined explicitly.

This concludes our discussion of T-dualities in the real basis. In the next section, we consider the same transformations in the complex basis, for later use. The reader interested in constructing solutions can however safely skip this technical discussion.

\section{$2.3 \quad$ T-dualities in the complex basis}

In this section we discuss the realisation of T-dualities in the complex basis defined by the central charge $Z=Z(\Gamma)$ and its Kähler derivative $Z_{a}=Z_{a}(\Gamma)$. The discussion here is parallel to the one of the previous section, in the real basis, and is complementary to it. However, the construction of the T-duality generators in the complex basis will be necessary to solve the first order equations describing black hole composites in the following.

For this purpose, we define the very small vector $R$ as in (2.17)-(2.18), while $R^{*}$ is defined from $R$ using an arbitrary phase, $e^{i \alpha} \neq \mathcal{N}[\Omega]$, as

$$
Z\left(R^{*}\right)=e^{3 i \alpha / 2} \mathcal{N}[\bar{\Omega}]^{1 / 2}, \quad Z_{a}\left(R^{*}\right)=e^{i \alpha / 2} \mathcal{N}[\bar{\Omega}]^{1 / 2} \Omega_{a},
$$


where the choice of the phases is done for later convenience. It will also be useful to define the complex function $Y$

$$
Y \equiv \frac{2}{1-e^{-i \alpha} \mathcal{N}[\Omega]}=-i \frac{e^{i \alpha / 2} \mathcal{N}[\bar{\Omega}]^{1 / 2}}{\operatorname{Im}\left(e^{i \alpha / 2} \mathcal{N}[\bar{\Omega}]^{1 / 2}\right)}=1+i e^{2 U} M,
$$

that has unit real part and the specific parametrisation of the imaginary part will become meaningful in the following. The vector (2.49) is by construction mutually nonlocal with $R$ because

$$
\left\langle R^{*}, R\right\rangle=\left(2 \operatorname{Im}\left(e^{i \alpha / 2} \mathcal{N}[\bar{\Omega}]^{1 / 2}\right)\right)^{3},
$$

and defines a natural magnetic dual to $R$.

We will first determine the T-dualities $\mathrm{T}^{+}$that leave $R$ invariant. We note that $\Omega_{a}$ is by construction (2.18) invariant with respect to a subgroup $K_{5} \subset K_{4}$. In order to describe the action of $\mathfrak{g}_{4}$ in (2.12) on this vector, we will parametrize the remaining $n_{v}-1$ generators of $\mathfrak{k}_{4}$, which describe the coset component $\mathfrak{k}_{4} \ominus \mathfrak{k}_{5}$, in terms of a vector $Q^{a}$. Requiring the matrix

$$
-G^{b}{ }_{a}(Q)=c_{a c e} c^{b d e} \Omega_{d} Q^{c}-\Omega_{a} Q^{b} Z_{b},
$$

to be anti-Hermitian and to preserve $\mathcal{N}[Z]$ fixes the relative coefficients and implies the constraints

$$
\Omega_{a} Q^{a}=0, \quad Q^{a}=\mathcal{N}[\bar{\Omega}] c^{a b c} \Omega_{b} \bar{Q}_{c} .
$$

Similarly, we parametrize $\mathfrak{u}(1)$ in $(2.12)$ by $\gamma$ and $\mathbb{C}^{n_{v}}$ by a complex vector $P^{a}$, such that the final result we find that the action of $\mathfrak{g}_{4}$ on a general vector reads

$$
\begin{aligned}
\delta Z & =P^{a} Z_{a}+3 i \gamma Z, \\
\delta Z_{a} & =\bar{P}_{a} Z+c_{a b c} P^{b} \bar{Z}^{c}+i \gamma Z_{a}+c_{a c e} c^{b d e} \Omega_{d} Q^{c} Z_{b}-\Omega_{a} Q^{b} Z_{b} .
\end{aligned}
$$

In order to describe T-dualities, we must impose that these transformations leave $R$ invariant, which can be shown to hold if ${ }^{8}$

$$
P^{a}=-i \gamma \mathcal{N}[\Omega] \bar{\Omega}^{a}-\mathcal{N}[\Omega] Q^{a} .
$$

One can now verify that the resulting transformations

$$
\begin{aligned}
\delta Z \equiv \mathrm{T}_{\gamma, Q}^{+} Z= & 3 i \gamma Z-i \gamma \mathcal{N}[\Omega] \bar{\Omega}^{a} Z_{a}-\mathcal{N}[\Omega] Q^{a} Z_{a} \\
\delta Z_{a} \equiv \mathrm{T}_{\gamma, Q}^{+} Z_{a}= & i \gamma Z_{a}+i \gamma \mathcal{N}[\bar{\Omega}] \Omega_{a} Z-i \gamma \mathcal{N}[\Omega] c_{a b c} \bar{\Omega}^{b} \bar{Z}^{c}-\mathcal{N}[\bar{\Omega}] \bar{Q}_{a} Z \\
& -\mathcal{N}[\Omega] c_{a b c} Q^{b} \bar{Z}^{c}+c_{a c e} c^{b d e} \Omega_{d} Q^{c} Z_{b}-\Omega_{a} Q^{b} Z_{b}
\end{aligned}
$$

leave $R$ invariant and commute with each other. Furthermore, one shows that $\mathrm{T}_{\gamma, Q}^{+}$is nilpotent of order four, as is clear from the example (2.3) where terms at most cubic in the

\footnotetext{
${ }^{8}$ Note that $R$ is also invariant if

$$
\gamma=Q^{a}=0, \quad P^{a} \Omega_{a}=0, \quad \bar{P}_{a}=-\mathcal{N}[\bar{\Omega}] c_{a b c} \bar{\Omega}^{b} P^{c} .
$$
}

These generators, along with the $K_{5}$ subgroup of $K_{4}$ and the generators described by (2.55), account for the full $G_{5} \ltimes \mathbb{R}^{n_{v}}$ subgroup of $G_{4}$ leaving invariant a given very small vector [32]. 
parameters $k^{i}$ appear. Indeed, $\mathrm{T}_{\gamma, Q}^{+}$can be identified with the corresponding generators in (2.35), which act as raising operators on the decomposition (2.29) and the vector $R$ is the highest weight vector, to which we assign weight 3 .

At this point it is important to appreciate the fact that, while we used the complex scalar dependent basis to define T-dualities, the following relations hold

$$
\mathrm{T}_{\gamma, Q}^{+} Z(\Gamma)=Z\left(\mathrm{~T}_{k}^{+} \Gamma\right), \quad \mathrm{T}_{\gamma, Q}^{+} Z_{a}(\Gamma)=Z_{a}\left(\mathrm{~T}_{k}^{+} \Gamma\right)
$$

where $\mathrm{T}_{k}^{+}$denote the representation of these generators in the real basis, parametrised in terms of a grade -1 vector $k$, as in (2.34)-(2.35). It follows that the parameters $\gamma$ and $Q^{a}$ depend on the $n_{v}$ constant parameters $k$ and the scalar fields.

As alluded to above, the second very small vector (2.49) plays a role dual to that of $R$, as one can check that $R^{*}$ is never a zero mode of the T-duality operator defined in (2.56), and in particular

$$
\begin{aligned}
Z\left(\mathrm{~T}_{k}^{+} R^{*}\right) & =Z(k)=-3 Y^{2} \mathcal{N}[\Omega] \gamma, \\
Z_{a}\left(\mathrm{~T}_{k}^{+} R^{*}\right) & =Z_{a}(k)=\left(3|Y|^{2}-2 Y\right) \Omega_{a} \gamma-4 i Y \bar{Q}_{a},
\end{aligned}
$$

where we also used (2.37) to given the explicit relation of the vector $k$ to the parameters $\gamma$ and $Q_{a}$. In addition, one can verify that $\left(\mathrm{T}_{\gamma, Q}^{+}\right)^{3} R^{*} \propto R$, as in $(2.41)$, so that the vector $R^{*}$ can be identified with the lowest weight vector of the operators $\mathrm{T}_{\gamma, Q}^{+}$, with assigned weight -3 .

As expected from the example in (2.3), the parameters $k^{i}$ must be a rank-three vector for a general T-duality. Indeed, the vector defined by (2.58) is of rank three, as can be shown by computing its quartic invariant. Furthermore, one can verify that this vector satisfies the reality constraint

$$
\bar{Z}^{a}-\mathcal{N}[\Omega] \bar{\Omega}^{a} \bar{Z}=e^{-i \alpha}\left(c^{a b c} \Omega_{b} Z_{c}+\bar{\Omega}^{a}\left(Z-\mathcal{N}[\Omega] \bar{\Omega}^{b} Z_{b}\right)\right),
$$

introduced in [14] in the study of single centre solutions. This defines a Lagrangian subspace that includes the small vector $R$, whereas one also verifies that

$$
\begin{aligned}
\bar{Z}\left(\mathrm{~T}^{+} R^{*}\right) & =e^{-2 i \alpha} Z\left(\mathrm{~T}^{+} R^{*}\right), \\
\bar{\Omega}^{a} Z_{a}\left(\mathrm{~T}^{+} R^{*}\right) & =\left(2 e^{-i \alpha}+\mathcal{N}[\bar{\Omega}]\right) Z\left(\mathrm{~T}^{+} R^{*}\right),
\end{aligned}
$$

which implies that $\left\langle\mathrm{T}^{+} R^{*}, R^{*}\right\rangle=0$.

Making use of the above structure based on the original very small vector $R$, one can proceed to define similar structures for the dual very small vector $R^{*}$, in exactly the same way. This seems redundant at first sight, since one can always identify the small vector invariant under the T-dualities with $R$, as above. However, this is more natural in view of the discussion in the real basis in the previous section, as well as for the applications we are interested in, where both vectors appear simultaneously. It is therefore useful to have a dual description in terms of $R^{*}$ throughout.

The T-dualities leaving $R^{*}$ invariant can then be shown to be defined as in (2.54) with parameters given by

$$
P^{a}=-e^{i \alpha}\left(i \gamma \bar{\Omega}^{a}+Q^{a}\right)
$$


where the $Q^{a}$ are again constrained by (2.53), so that the resulting transformations read

$$
\begin{aligned}
\delta Z \equiv \mathrm{T}_{\gamma, Q}^{-} Z= & 3 i \gamma Z-i \gamma e^{i \alpha} \bar{\Omega}^{a} Z_{a}-e^{i \alpha} Q^{a} Z_{a} \\
\delta Z_{a} \equiv \mathrm{T}_{\gamma, Q}^{-} Z_{a}= & i \gamma e^{-i \alpha} \Omega_{a} Z-i \gamma e^{i \alpha} c_{a b c} \bar{\Omega}^{b} \bar{Z}^{c}+i \gamma Z_{a}-e^{-i \alpha} \bar{Q}_{a} Z \\
& -e^{i \alpha} c_{a b c} Q^{b} \bar{Z}^{c}+c_{a c e} c^{b d e} \Omega_{d} Q^{c} Z_{b}-\Omega_{a} Q^{b} Z_{b} .
\end{aligned}
$$

As expected, $R$ is never invariant under (2.62), with transformation rule

$$
\begin{aligned}
Z\left(\mathrm{~T}_{k}^{-} R\right) & =Z(k)=-6 i \frac{e^{i \alpha}}{Y} \gamma \\
Z_{a}\left(\mathrm{~T}_{k}^{-} R\right) & =Z_{a}(k)=-2 i|Y|^{-2}(\bar{Y}-2 Y) \Omega_{a} \gamma+4|Y|^{-2} \bar{Q}_{a},
\end{aligned}
$$

where we show the relation of the vector $k$ in (2.37) to the parameters in the complex basis. Again, one can compute that $\left(\mathrm{T}_{\gamma, Q}^{-}\right)^{3} R \propto R^{*}$, consistent with (2.43). It follows that $\mathrm{T}_{\gamma, Q}^{-}$are lowering operators with $R^{*}$ and $R$ as their lowest and highest weight vectors respectively. Furthermore, one can define a Lagrangian subspace that includes $R^{*}$ and $\mathrm{T}^{-} R$, through the constraint dual to (2.59), as

$$
\bar{Z}^{a}-e^{i \alpha} \bar{\Omega}^{a} \bar{Z}=\mathcal{N}[\bar{\Omega}] c^{a b c} \Omega_{b} Z_{c}+\bar{\Omega}^{a}\left(e^{-i \alpha} Z-\bar{\Omega}^{b} Z_{b}\right) .
$$

In addition, the vector (2.63) satisfies the relations

$$
\begin{aligned}
\bar{Z}\left(\mathrm{~T}^{-} R\right) & =e^{-i \alpha} \mathcal{N}[\bar{\Omega}] Z\left(\mathrm{~T}^{-} R\right), \\
\bar{\Omega}^{a} Z_{a}\left(\mathrm{~T}^{-} R\right) & =\left(2 \mathcal{N}[\bar{\Omega}]+e^{-i \alpha}\right) Z\left(\mathrm{~T}^{-} R\right),
\end{aligned}
$$

which imply that $\left\langle\mathrm{T}^{-} R, R\right\rangle=0$.

One can obtain the action of the relevant generator $\mathbf{h}_{T}$ defined in (2.24) in the complex basis, as the commutator of a T-duality leaving $R$ invariant and a T-duality leaving $R^{*}$ invariant. In general, such a commutator will also give rise to an element of the grade zero component $\mathfrak{g}_{5}$, as in (2.33). Choosing the parameters $\gamma$ and $Q^{a}$ for the two transformations to be identical, one obtains

$$
\left[\mathrm{T}^{+}, \mathrm{T}^{-}\right]=\mathbf{G}\left(\gamma, Q^{a}\right),
$$

where $\mathbf{G}\left(\gamma, Q^{a}\right)$ is a generator of $\mathfrak{g l}_{1} \oplus \mathfrak{g}_{5}$ bilinear in $\gamma$ and $Q^{a}$. The $\mathfrak{g l}_{1}$ component, which is to be identified with the operator $\mathbf{h}_{T}$, corresponds to the transformation of parameter $\frac{1}{2} \gamma^{2}+\frac{1}{3} Q^{a} \bar{Q}_{a}$, whereas the $\mathfrak{g}_{5}$ transformation is parametrised by $\gamma Q^{a}$ and $Q^{a} \bar{Q}_{b}-\frac{1}{n_{v}-1}\left(\delta_{b}^{a}-\frac{1}{3} \bar{\Omega}^{a} \Omega_{b}\right) Q^{c} \bar{Q}_{c}$. To project to the $\mathfrak{g l}_{1}$ component, one can simply identify the terms quadratic in $Q^{a}$ as

$$
Q^{a} \bar{Q}_{b} \sim \frac{1}{n_{v}-1}\left(\delta_{b}^{a}-\frac{1}{3} \bar{\Omega}^{a} \Omega_{b}\right) Q^{c} \bar{Q}_{c},
$$

so as to cancel all the terms in $\mathfrak{g}_{5}$. Equivalently, this identification can be understood as an average obtained by acting on the parameter $Q^{a}$ with the $K_{5} \subset K_{4}$ subgroup leaving $\Omega_{a}$ invariant, and integrating out the result over $K_{5}$. By definition, none of the generators of $\mathfrak{g}_{5}$ 
are $K_{5}$ singlets, and the resulting expression is necessarily proportional to the $\mathfrak{g l}_{1}$ generator $\mathbf{h}_{T}$. Because of the reality constraint (2.53) on the $Q^{a}$, this average furthermore implies

$$
Q^{a} Q^{b} \sim \frac{1}{n_{v}-1} \bar{Q}_{d} Q^{d}\left(\mathcal{N}[\bar{\Omega}] c^{a b c} \Omega_{c}-\frac{2}{3} \bar{\Omega}^{a} \bar{\Omega}^{b}\right),
$$

and therefore

$$
c_{a b c} Q^{b} Q^{c}=\frac{1}{3} \bar{Q}_{b} Q^{b} \mathcal{N}[\bar{\Omega}] \Omega_{a},
$$

where we used (2.11) to show that $c^{a c d} c_{b c d}=\frac{n_{v}+3}{3} \delta_{b}^{a}$. In practice, (2.69) is the only constraint one needs to use when computing the commutator in (2.66).

After imposing the relations above, one finds

$$
\begin{aligned}
\mathbf{h}_{T} Z= & \frac{1}{4}|Y|^{2}\left(3\left(e^{i \alpha} \mathcal{N}[\bar{\Omega}]-e^{-i \alpha} \mathcal{N}[\Omega]\right) Z+2\left(\mathcal{N}[\Omega]-e^{i \alpha}\right) \bar{\Omega}^{a} Z_{a}\right) \\
\mathbf{h}_{T} Z_{a}= & \frac{1}{4}|Y|^{2}\left(\left(e^{i \alpha} \mathcal{N}[\bar{\Omega}]-e^{-i \alpha} \mathcal{N}[\Omega]\right) Z_{a}+2\left(\mathcal{N}[\bar{\Omega}]-e^{-i \alpha}\right) Z \Omega_{a}\right. \\
& \left.+2\left(\mathcal{N}[\Omega]-e^{i \alpha}\right) c_{a b c} \bar{\Omega}^{b} \bar{Z}^{c}\right) .
\end{aligned}
$$

One can now check that (2.44) is indeed satisfied in the complex basis. Similarly, the vector space of charges splits into 4 subspaces of eigenvalue $\{-3,-1,1,3\}$ with respect to this linear operator.

By construction, $R$ and $R^{*}$ are the unique vectors of eingenvalue 3 and -3 respectively, up to an overall rescaling. The remaining $2 n_{v}$ directions can then be simply identified with the parameters of the two T-dualities $\mathrm{T}^{ \pm}$, as given above. It follows that the eigenspace of eigenvalue -1 is spanned by the $n_{v}$ vectors that satisfy the constraint (2.59) and are mutually local ${ }^{9}$ with $R^{*}$, as $\left\langle R^{*}, \Gamma^{(-1)}\right\rangle=0$, which is equivalent to

$$
\begin{aligned}
\bar{Z}\left(\Gamma^{(-1)}\right) & =e^{-2 i \alpha} Z\left(\Gamma^{(-1)}\right), \\
\bar{\Omega}^{a} Z_{a}\left(\Gamma^{(-1)}\right) & =\left(2 e^{-i \alpha}+\mathcal{N}[\bar{\Omega}]\right) Z\left(\Gamma^{(-1)}\right), \\
c^{a b c} \Omega_{b} Z_{c}\left(\Gamma^{(-1)}\right) & =e^{i \alpha} \bar{Z}^{a}\left(\Gamma^{(-1)}\right)+e^{-i \alpha} \mathcal{N}[\Omega] \bar{\Omega}^{a} Z\left(\Gamma^{(-1)}\right) .
\end{aligned}
$$

Similarly, the eigenspace of eigenvalue 1 is spanned by the $n_{v}$ vectors that satisfy the dual constraint (2.64) and $\left\langle R, \Gamma^{(1)}\right\rangle=0$, which lead to

$$
\begin{aligned}
\bar{Z}\left(\Gamma^{(1)}\right) & =e^{-i \alpha} \mathcal{N}[\bar{\Omega}] Z\left(\Gamma^{(1)}\right), \\
\bar{\Omega}^{a} Z_{a}\left(\Gamma^{(1)}\right) & =\left(2 \mathcal{N}[\bar{\Omega}]+e^{-i \alpha}\right) Z\left(\Gamma^{(1)}\right), \\
c^{a b c} \Omega_{b} Z_{c}\left(\Gamma^{(1)}\right) & =\mathcal{N}[\Omega] \bar{Z}^{a}\left(\Gamma^{(1)}\right)+\bar{\Omega}^{a} Z\left(\Gamma^{(1)}\right) .
\end{aligned}
$$

These equations can be identified from (2.30) in the real basis as (2.31).

\section{The $c^{*}$-map, nilpotent orbits and first order systems}

In this section we consider the first order systems describing multi-centre non-BPS black holes in $\mathcal{N}=2$ supergravity coupled to $n_{v}$ vector multiplets labelled by an index $i=$

\footnotetext{
${ }^{9}$ This is has to be imposed since $R$ itself is the trivial solution of (2.59).
} 
$1, \ldots, n_{v}$. We refer to the appendix for a short overview of our conventions on $\mathcal{N}=2$ supergravity, which coincide with the ones in [14], to which we refer for further details.

The systems of black holes we are interested in can be constructed systematically in the special case when the special Kähler manifold, $\mathcal{M}_{4}$, parametrised by the vector multiplet scalars, $t^{i}$, is symmetric. Moreover, we exclusively consider stationary solutions, i.e. we always assume a timelike isometry. In this case, one can consider a timelike dimensional reduction to three dimensions and dualise all vector fields to scalars [33], to obtain an effective euclidean sigma model describing stationary black hole backgrounds. The resulting equations of motion are still rather complicated, so that it is common to consider special linear systems that solve the full equations of motion, but do not provide a full list of possible solutions. There are two such systems known, namely the composite non-BPS system [12] and the almost-BPS system [15], which together account for a representative majority of the explicitly known multi-centre solutions featuring a flat three-dimensional base space. The purpose of this section is to define these systems in terms of four-dimensional, manifestly duality covariant quantities, aiming for a clear description of their general structure.

To this end, we make use of the duality symmetries of the three-dimensional theory resulting from the dimensional reduction, through the formalism developed in [14, 34]. We therefore first describe the basics of this effective theory in section 3.1, followed by a discussion of the method of nilpotent orbits in section 3.2. We then present the derivation of the first order flow equations for the composite non-BPS system and the almost-BPS system in sections 3.3 and 3.4 respectively. Note that, while the derivation of the almostBPS system has not appeared before, our section 3.3 is essentially a review of the derivation of the same system in [14], which we include for completeness.

\subsection{The three-dimensional non-linear sigma model}

In order to describe stationary asymptotically flat extremal black holes, we introduce the standard Ansatz for the metric

$$
d s^{2}=-e^{2 U}(d t+\omega)^{2}+e^{-2 U} d \mathbf{x} \cdot d \mathbf{x},
$$

in terms of a scale function $U(x)$ and the Kaluza-Klein one-form $\omega(x)$ (with spatial components only), which are both required to asymptote to zero at spatial infinity. Here and henceforth, all quantities are independent of time, so that all scalars and forms are defined on the flat three-dimensional base. The $n_{v}+1$ gauge fields of the theory $F^{I}=d A^{I}$ for $I=0, \ldots n_{v}$ include the graviphoton and the vector multiplet gauge fields. Together with their magnetic duals, these can be arranged in a symplectic vector, $\mathcal{F}$, as in (A.2), which transforms linearly under electric/magnetic duality. For a background as in (3.1), the appropriate decomposition of the gauge fields takes the form

$$
2 \mathcal{A}=\zeta(d t+\omega)+w
$$

and accordingly for the field strengths

$$
2 \mathcal{F}=d \zeta(d t+\omega)+F, \quad F=\zeta d \omega+d w,
$$


where we defined the gauge field scalars $\zeta$, arising as the time component of the gauge fields, and the one-forms $w$ describing the charges. Here, $F$ is defined as the spatial component of the field strength, which is not closed but satisfies

$$
d F=e^{2 U} \star d \omega \wedge \mathrm{J} F
$$

according to (A.15), which can be written as

$$
d \zeta=e^{2 U} \mathrm{~J} \star F
$$

Note that this first order equation determines the $\zeta$ in terms of the vector fields $w$ and the scalars.

Upon dimensional reduction over the time direction, the set of moduli $t^{i}$ parametrising the coset space (2.7) are extended to include the scaling factor $U$ and the scalar dual to the angular momentum $\omega$ in (3.1), as well as the fields $\zeta$ in (3.3), which altogether parametrize the para-quaternionic symmetric space ${ }^{10}$

$$
\mathcal{M}_{3} \cong G_{3} /\left(\mathrm{SL}(2) \times G_{4}\right)
$$

This defines the so-called $c^{*}$-map, which can be related to the standard $c$-map [35] by analytic continuation. The three-dimensional symmetry group Lie algebra $\mathfrak{g}_{3}$ decomposes as

$$
\mathfrak{g}_{3} \cong \mathbf{1}^{(-2)} \oplus \mathfrak{l}_{4}^{(-1)} \oplus\left(\mathfrak{g l}_{1} \oplus \mathfrak{g}_{4}\right)^{(0)} \oplus \mathfrak{l}_{4}^{(1)} \oplus \mathbf{1}^{(2)}
$$

where the weights refer to the eigenvalues under the adjoint action of the $\mathfrak{g l}_{1}$ generator. The grade one generators in $\mathfrak{r}_{4}^{(1)}$ are associated to the gauge invariance with respect to a constant shift of the scalars $\zeta$, and accordingly the grade two generator corresponds to the shift of integration constant defining the scalar dual to $\omega$.

As discussed in more detail in [14], the equations of motion for the scalar fields parametrising the symmetric space $\mathcal{M}_{3}$ are expressed in terms of the corresponding Maurer-Cartan form

$$
v^{-1} d v=P+B
$$

Here, $v \in G_{3}$ is a coset representative describing the scalar fields, while $P$ is the coset component of the Maurer-Cartan form, defining the scalar momenta. Similarly, $B$ is the $\mathfrak{s l}_{2} \oplus \mathfrak{g}_{4}$ component defining the pulled back spin connection. In components, the scalar momenta are defined as

$$
\mathrm{w} \equiv-d U-\frac{i}{2} e^{2 U} \star d \omega, \quad \Sigma^{a}=-e_{i}^{a} d t^{i}, \quad Z \equiv e^{U} Z(\star F), \quad Z_{a} \equiv e^{U} Z_{a}(\star F),
$$

where we introduce some shorthand notations, based on the central charges in (A.11), that will be used for the remainder of the section.

At this stage it is important to introduce some properties of the $\mathfrak{g}_{3}$ algebra. The components of an element of the Lie algebra $\mathfrak{g}_{3}$ in the coset component $\mathfrak{g}_{3} \ominus\left(\mathfrak{s l}_{2} \oplus \mathfrak{g}_{4}\right)$,

\footnotetext{
${ }^{10}$ Here para-quaternionic refers to the property that the holonomy group of $\mathcal{M}_{3} \operatorname{SL}(2) \times G_{4} \subset \operatorname{SL}(2) \times$ $\operatorname{Sp}\left(2 n_{v}+2, \mathbb{R}\right)$.
} 
as given in (3.9) above, correspond to $\mathrm{U}(1) \times \mathrm{U}(1) \times K_{4}$ irreducible representations as the two complex parameters $\mathrm{w}$ and $Z$ and the two complex vectors $\bar{Z}^{a}, \Sigma^{a}$ transform in the $\mathbb{C}^{n_{v}}$ representation of $K_{4}$, according to the decomposition (2.12), the same as the scalar field momenta $e_{i}^{a} d t^{i}$ in four dimensions. As one can check explicitly, the quadratic trace invariant which defines the $\mathrm{SL}(2) \times G_{4}$ invariant norm

$$
|\mathrm{w}|^{2}-|Z|^{2}-Z_{a} \bar{Z}^{a}+\Sigma^{a} \bar{\Sigma}_{a}
$$

is equivalent to the effective Lagrangian in the background (3.1).

The $\mathfrak{s l}_{2}$ algebra is realised on these components as

$$
\delta \mathrm{w}=i \rho \mathrm{w}+\bar{\lambda} Z, \quad \delta Z=-i \rho Z+\lambda \mathrm{w}, \quad \delta \bar{Z}^{a}=i \rho \bar{Z}^{a}+\bar{\lambda} \Sigma^{a}, \quad \delta \Sigma^{a}=-i \rho \Sigma^{a}+\lambda \bar{Z}^{a},
$$

where $\rho$ and $\lambda$ are a real and a complex parameter respectively, parametrising the $\mathfrak{s l}_{2}$ group. Similarly, the action of $\mathfrak{g}_{4}$ can be written as using the parameters defined below (2.12), as

$$
\begin{array}{ll}
\delta \mathrm{w}=\Lambda_{a} \bar{Z}^{a}+3 i \gamma \mathrm{w}, & \delta \Sigma^{a}=\bar{\Lambda}^{a} Z+c^{a b c} \Lambda_{b} Z_{c}+G^{a}{ }_{b} \Sigma^{b}+i \gamma \Sigma^{a}, \\
\delta Z=\Lambda_{a} \Sigma^{a}+3 i \gamma Z, & \delta \bar{Z}^{a}=\bar{\Lambda}^{a} \mathrm{w}+c^{a b c} \Lambda_{b} \bar{\Sigma}_{c}+G^{a}{ }_{b} \bar{Z}^{b}+i \gamma \bar{Z}^{a} .
\end{array}
$$

Note that the $\mathfrak{g}_{4}$ action defined by these equations corresponds to the divisor group in (3.6), rather than the original $\mathfrak{g}_{4}$ as given in four dimensions in (2.13), the two being related by a conjugation in $\mathfrak{g}_{3}$.

Finally, we give the components of the $\mathfrak{s l}_{2} \oplus \mathfrak{g}_{4}$ component of the Maurer-Cartan form, $B$, along $\mathfrak{s l}_{2}$

$$
\rho(B)=-\frac{1}{4} \mathrm{e}^{2 U} \star d \omega-\frac{1}{2} Q, \quad \lambda(B)=e^{U} Z(\star F),
$$

and along $\mathfrak{g}_{4}$

$$
\begin{aligned}
\gamma(B) & =-\frac{1}{4} \mathrm{e}^{2 U} \star d \omega+\frac{1}{6} Q, \quad \Lambda_{a}(B)=e^{U} Z_{a}(\star F), \\
G^{a}{ }_{b}(B) & =e_{i}^{a} \partial_{\bar{\jmath}} e_{b}^{i} d \bar{t}^{\bar{\jmath}}-e_{\bar{\jmath} b} \partial_{i} e^{\bar{\jmath} a} d t^{i}-\frac{2 i}{3} \delta_{b}^{a} Q,
\end{aligned}
$$

where $G^{a}{ }_{b}(B)$ defines the $\mathfrak{k}_{4}$ valued traceless ${ }^{11}$ component of the pulled back spin connection on $\mathcal{M}_{4}$ and $Q$ is the pulled back Kähler connection (A.8).

\section{$3.2 \quad$ Nilpotent orbits}

The basic observation for constructing black hole solutions in four dimensions using the three-dimensional Euclidean theory describing stationary solutions is that regular stationary solutions of $\mathcal{N}=2$ supergravity with a flat three-dimensional base metric can be described by a three-dimensional momentum $P$ that is nilpotent as a Lie algebra element. This implies in particular that $P$ can be written in terms of the basis element $\mathbf{e}_{\alpha}$ of a nilpotent subalgebra of $\mathfrak{g}_{3}$. Such a subalgebra is always associated to a semi-simple element ${ }^{12}$ $\mathbf{h}$ of $\mathfrak{s l}_{2} \oplus \mathfrak{g}_{4}$ such that

$$
\mathbf{h} \mathbf{e}_{\alpha}:=\left[\mathbf{h}, \mathbf{e}_{\alpha}\right]=p_{\alpha} \mathbf{e}_{\alpha}, \quad 1 \leq p_{\alpha} \leq n,
$$

\footnotetext{
${ }^{11}$ To prove that $G^{a}{ }_{b}(B)$ is indeed traceless, one can use (2.10) and (2.11) to show that $c^{a c d} c_{b c d}=\frac{n_{v}+3}{3} \delta_{b}^{a}$.

${ }^{12} \mathrm{Semi}$-simple means that it is in the conjugation class of an element of the Cartan subalgebra.
} 
where $n$ defines the maximal possible eigenvalue of $\operatorname{ad}_{\mathbf{h}}$ in $\mathfrak{g}_{3}$. This implies for instance the equation

$$
\prod_{i=1}^{n}(\mathbf{h}-i) P=0
$$

which defines a first order constraint on the components of $P$. In order for (3.17) to be consistent with the equations of motion and the Bianchi identity, the covariant derivative of the generator $\mathbf{h}$ must satisfy

$$
\sum_{i=1}^{n} \prod_{j=i+1}^{n}(\mathbf{h}-j) d_{B} \mathbf{h} \prod_{k=1}^{i-1}(\mathbf{h}-k) \wedge P=0, \quad \sum_{i=1}^{n} \prod_{j=i+1}^{n}(\mathbf{h}-j) d_{B} \mathbf{h} \prod_{k=1}^{i-1}(\mathbf{h}-k) \star P=0 .
$$

These equations are satisfied if $d_{B} \mathbf{h}$ also lies in the nilpotent algebra defined by $\mathbf{h}$, or equivalently if one imposes that

$$
\prod_{i=1}^{n}\left(\operatorname{ad}_{\mathbf{h}}-i\right) d_{B} \mathbf{h}=0
$$

Note that this is not necessarily the most general solution for the generator $\mathbf{h}$, as it might be possible to construct special solutions, for which e.g. there are preferred spacetime directions described by a nonzero derivative. Considering this type of solutions can be understood as the natural generalisation of the BPS black hole solutions in $[6,7]$ to include supersymmetric string solutions. These are not accounted for by the standard black hole ansatz, but are are allowed by the requirement of preserved supersymmetry. Similarly, we will only consider the generic situation in (3.19), which should be satisfied for all composite black hole solutions with a flat three-dimensional base.

Without loss of generality, one can always choose the generators $\mathbf{h} \in \mathfrak{s l}_{2} \oplus \mathfrak{g}_{4}$ such that only its components $\lambda$ and $\Lambda_{a}$ do not vanish, as these are the only generators with a positive Cartan norm. ${ }^{13}$ Equation (3.19) can then be viewed as first order equations for these auxiliary components, which can be solved to determine their evolution in space in terms of the physical fields. With this information at hand, equation (3.17) then defines first order equations for the physical fields, which contain the auxiliary components $\lambda$ and $\Lambda_{a}$ and determine $\mathrm{w}$ and $\Sigma^{a}$ of (3.9) in terms of $e^{U} Z(\star F)$ and $e^{U} Z_{a}(\star F)$, plus some possible constraints on the latter if the dimension of the coset component of the nilpotent algebra defined by $\mathbf{h}$ is strictly less than $2 n_{v}+2$.

Given the definitions in the previous section, it is possible to make (3.17)-(3.19) explicit, in order to determine the auxiliary components and the first order flow directly. A simple example is given by the BPS system, which is characterised by an element of $\mathbf{h}_{*} \in \mathfrak{s l}_{2}$, i.e. $\Lambda_{a}\left(\mathbf{h}_{*}\right)=0$. It follows that its only nonvanishing component is $\lambda\left(\mathbf{h}_{*}\right)=e^{i \alpha}$, where this phase is identified with the phase $\alpha$ that defines the covariantly constant spinors as in [6]. The action of this generator follows from (3.11) as

$$
\mathbf{h}_{*} \mathrm{w}=e^{-i \alpha} Z, \quad \mathbf{h}_{*} Z=e^{i \alpha} \mathrm{w}, \quad \mathbf{h}_{*} \bar{Z}^{a}=e^{-i \alpha} \Sigma^{a}, \quad \mathbf{h}_{*} \Sigma^{a}=e^{i \alpha} \bar{Z}^{a},
$$

\footnotetext{
${ }^{13}$ This is similar to the canonical choice of $\partial / \partial t$ as a timelike vector in Minkowski spacetime, i.e. there are more general timelike vectors but they can be obtained from the canonical one by symmetries.
} 
while the relevant eigenvalue equation (3.17) is simply

$$
\mathbf{h}_{*} P=P .
$$

The last relation clearly imposes a linear relation between the derivatives of the fourdimensional scalars and the gauge fields upon using (3.20). The phase $e^{i \alpha}$ is determined by (3.19), which can be shown to reduce to

$$
d \alpha+Q+\frac{1}{2} e^{2 U} \star d \omega+2 \operatorname{Im}\left(e^{-i \alpha} Z\right)=d \alpha+Q-\frac{1}{2} e^{2 U} \star d \omega=0,
$$

where in the second equality we used the first of (3.20) and (3.21). These equations are easily seen to be equivalent to the BPS system of $[6,7]$ and we refer to [34] for a more detailed analysis of the eigenvalue equation (3.16) for this generator, leading to the system of equations describing multi-centre BPS black holes. Similarly, non-BPS solutions with vanishing central charge at the horizons are described in a similar fashion, with $\lambda=0$ and a normalised rank one $\Lambda_{a}$ (i.e. $c^{a b c} \Lambda_{b} \Lambda_{c}=0$ and $\Lambda_{a} \bar{\Lambda}^{a}=1$ ) [34].

Other examples of such constructions using nilpotent orbits have been used to obtain the systems describing respectively single centre non-BPS black holes with a non-vanishing central charge at the horizon and the composite non-BPS multi-centre system [14]. In the next two sections we discuss in some detail the construction of the two multi-centre non-BPS systems, the composite non-BPS and the almost-BPS, which form the basis of this paper.

\subsection{Composite non-BPS flows}

The composite non-BPS system [12-14], describes configurations of interacting non-BPS centres and corresponds to an eigenvalue equation as in (3.16), where the relevant element $\mathbf{h}_{\mathrm{C}}$ belongs to $\mathfrak{g}_{4}$. As the scalar momentum $P$ must be of positive grade with respect to $\mathbf{h}_{\mathrm{C}}$ (given that $p_{\alpha}>0$ ), we consider the relevant decomposition of the coset component, parametrising $\mathbf{2} \otimes \mathbb{R}^{2 n_{v}+2}$ through (3.9), in terms of grades with respect to an element $\mathbf{h}_{\mathrm{C}} \in \mathfrak{g}_{4}$. The relevant graded decomposition is in this case

$$
\mathfrak{g}_{4} \cong\left(\mathbb{R}^{n_{v}}\right)^{(-2)} \oplus\left(\mathfrak{g l}_{1} \oplus \mathfrak{g}_{5}\right)^{(0)} \oplus\left(\mathbb{R}^{n_{v}}\right)^{(2)},
$$

for $\mathfrak{g}_{4}$ itself and

$$
\mathbf{2} \otimes \mathbb{R}^{2 n_{v}+2} \cong \mathbf{2}^{(-3)} \oplus\left(\mathbf{2} \otimes \mathbb{R}^{n_{v}}\right)^{(-1)} \oplus\left(\mathbf{2} \otimes \mathbb{R}^{n_{v}}\right)^{(1)} \oplus \mathbf{2}^{(3)},
$$

for the coset component, i.e. $P \in\left(\mathbf{2} \otimes \mathbb{R}^{n_{v}}\right)^{(1)} \oplus \mathbf{2}^{(3)}$. We can choose $\mathbf{h}_{\mathrm{C}}$ to be Hermitian (i.e. to lie in $\mathfrak{g}_{4} \ominus\left(\mathfrak{u}(1) \oplus \mathfrak{k}_{4}\right)$ ), so that it is realised for

$$
\gamma\left(\mathbf{h}_{\mathrm{C}}\right)=0, \quad \Lambda_{a}\left(\mathbf{h}_{\mathrm{C}}\right)=\Omega_{a}, \quad G_{b}^{a}\left(\mathbf{h}_{\mathrm{C}}\right)=0,
$$

where $\Omega_{a}$ describes a very small vector, as in (2.18). Equivalently, $\Omega_{a}$ is in the $\mathrm{U}(1) \times K_{4}$ orbit of the Jordan algebra identity.

More explicitly, one finds the following action on the coset component

$$
\mathbf{h}_{\mathrm{C}} \mathrm{W}=\Omega_{a} \bar{Z}^{a} \quad \mathbf{h}_{\mathrm{C}} Z=\Omega_{a} \Sigma^{a} \quad \mathbf{h}_{\mathrm{C}} \bar{Z}^{a}=\bar{\Omega}^{a} \mathrm{w}+c^{a b c} \Omega_{b} \bar{\Sigma}_{c} \quad \mathbf{h}_{\mathrm{C}} \Sigma^{a}=\bar{\Omega}^{a} Z+c^{a b c} \Omega_{b} Z_{c},
$$


which is to be identified with the elements of the coset component, through (3.16) for $p_{\alpha}=1$ and $p_{\alpha}=3$, according to (3.24). Considering a general linear combination of the grade one and three solutions as in [14], one can express $\mathrm{w}$ and $\Sigma^{a}$ in terms of $Z$ and $Z_{a}$ as

$$
\mathrm{w}=\frac{1}{2}\left(\Omega_{a} \bar{Z}^{a}-\mathcal{N}[\Omega] \bar{Z}\right), \quad \Sigma^{a}=c^{a b c} \Omega_{b} Z_{c}+\frac{1}{2} \bar{\Omega}^{a}\left(Z-\mathcal{N}[\Omega] \bar{\Omega}^{b} Z_{b}\right),
$$

which are the explicit first order relations for the scalar momenta, upon using the definitions in (3.9).

These flow equations contain the auxiliary components $\mathcal{N}[\Omega]$ and $\Omega_{a}$ of $\mathbf{h}_{\mathrm{C}}$, which satisfy (2.18) and define a very small vector $R$ of unit mass through

$$
Z(R)=\mathcal{N}[\Omega] \quad Z_{a}(R)=\Omega_{a} .
$$

The flow equations for these auxiliary fields are given by (3.19), which in this system reduces to

$$
\left[\mathbf{h}_{\mathrm{C}}, d_{B} \mathbf{h}_{\mathrm{C}}\right]=2 d_{B} \mathbf{h}_{\mathrm{C}},
$$

in view the fact that $d_{B} \mathbf{h}_{C}$ lies in $\mathfrak{g}_{4}$ and is inert under $\mathfrak{g}_{5}$ by definition, and the decomposition in (3.23). Using the explicit form of $B$ in (3.13)-(3.15) and the first order flow (3.27), one computes the components of $d_{B} \mathbf{h}$ as

$$
\begin{aligned}
\gamma\left(d_{B} \mathbf{h}\right) & =\frac{2}{3} \operatorname{Im}\left[\bar{\Omega}^{a} Z_{a}\right] \\
\Lambda_{a}\left(d_{B} \mathbf{h}\right) & =Z_{a}(d R)+\operatorname{Re}\left[\mathcal{N}[\bar{\Omega}] \Omega_{b} e_{i}^{b} d t^{i}\right] Z_{a}(R)-\left(Z_{a}+\mathcal{N}[\Omega] c_{a b c} \bar{\Omega}^{b} \bar{Z}^{c}-\Omega_{a} \Omega_{b} \bar{Z}^{b}\right) \\
G^{a}{ }_{b}\left(d_{B} \mathbf{h}\right) & =c^{a c e} c_{b d e}\left(\bar{\Omega}^{d} Z_{c}-\Omega_{c} \bar{Z}^{d}\right)+\Omega_{b} \bar{Z}^{a}-\bar{\Omega}^{a} Z_{b}-\frac{2 i}{3} \delta_{b}^{a} \operatorname{Im}\left[\bar{\Omega}^{c} Z_{c}\right]
\end{aligned}
$$

where we explicitly separated the terms depending on the derivative of the vector $R$. It is now straightforward (though cumbersome) to evaluate (3.29), imposing the above relations and

$$
\begin{aligned}
c^{a c e} c_{b d e} \bar{\Omega}^{d}\left(\mathcal{N}[\Omega] c_{c f g} \bar{\Omega}^{f} \bar{Z}^{g}-\Omega_{c} \Omega_{f} \bar{Z}^{f}\right)-\bar{\Omega}^{a}\left(\mathcal{N}[\Omega] c_{b c d} \bar{\Omega}^{c} \bar{Z}^{d}-\Omega_{b} \Omega_{c} \bar{Z}^{c}\right) \\
=-c^{a c e} c_{b d e} \Omega_{c} \bar{Z}^{d}+\Omega_{b} \bar{Z}^{a},
\end{aligned}
$$

which can be shown using (2.11). The result one finds is that (3.29) is identically satisfied for (3.30), if and only if the combination of the first two terms in $\Lambda_{a}\left(d_{B} \mathbf{h}\right)$ vanishes. This condition implies [14] that there exists a constant symplectic vector $\hat{R}$, since

$$
d R=-\operatorname{Re}\left[\mathcal{N}[\bar{\Omega}] \Omega_{b} e_{i}^{b} d t^{i}\right] R, \quad \Rightarrow \quad R=\frac{\hat{R}}{|Z(\hat{R})|},
$$

where the second equation follows from the first by use of standard special geometry identities. It follows that the generator $\mathbf{h}_{\mathrm{C}}$ is in this case determined by a constant very small projective vector $\hat{R}$ and the scalar fields are such that

$$
\Lambda_{a}\left(\mathbf{h}_{\mathbf{c}}\right)=\frac{Z_{a}(\hat{R})}{|Z(\hat{R})|} .
$$


One can now return to (3.27), which becomes a first order flow equation for the scalars $d U, \star d \omega$ and $d t^{i}$ in terms of the gauge fields and the constant vector $\hat{R}$. Further details and the characterisation of solutions to these equations are given in section 4 .

A special case of this system, namely the single centre subclass, was discussed in detail in [14]. Indeed, one expects a system describing multi-centre black hole solutions to contain a consistent subsystem describing single centre black holes. In the case at hand, one can restrict the above equations (3.27) to this subsystem by imposing that the scalar momentum is also of positive grade with respect to the generator $\frac{1}{2}\left(\mathbf{h}_{\mathrm{C}}+\mathbf{h}_{*}\right)$ where $\mathbf{h}_{\mathrm{C}}$ and $\mathbf{h}_{*}$ are the generators that define the composite system through (3.26) and the BPS system through (3.20) respectively. The result one obtains is that the single centre nonBPS momenta satisfy (3.27), for $Z$ and $Z_{a}$ constrained to satisfy exactly the same phase dependent projection (2.59) above,

$$
\bar{Z}^{a}-\mathcal{N}[\Omega] \bar{\Omega}^{a} \bar{Z}=e^{-i \alpha}\left(c^{a b c} \Omega_{b} Z_{c}+\bar{\Omega}^{a}\left(Z-\mathcal{N}[\Omega] \bar{\Omega}^{b} Z_{b}\right)\right)
$$

which represents a constraint on the physical degrees of freedom that is necessary to reduce to single centre solutions. One can interpret this constraint in terms of the decomposition in (2.29), upon identifying the very small vector $\hat{R}$ in (3.33) with the vector $R$ in section 2.2. It follows that (3.34) implies that only the grade $(-1)$ and $(+3)$ components of the gauge fields (and thus charges) are allowed. We refrain from discussing the single centre system, as we will deal with the multi-centre case in what follows. However, we will sometimes make use of structures present already in the single centre case, referring to [14] for an in depth discussion.

\subsection{Almost-BPS flows}

We now turn to the derivation of the first order flow equations for the almost-BPS system [15] of multi-centre black hole solutions. Our treatment is purely in terms of four/three dimensional quantities, but we still refer to this system as almost-BPS, as we will find that it contains the original almost-BPS equations, derived from the BPS conditions of five dimensional supergravity. However, the five dimensional system is only defined in a fixed frame and does not allow for generic charges at the centres, while we will present a manifestly duality covariant form of these equations, as we did for the composite non-BPS system in the previous section. In this sense, the almost-BPS system below is larger, as it contains the one in five dimensions, as well as all the charge configurations that can be obtained from it by dualities. Our covariant system also represents the general form of the almostBPS system of [12], where a frame was chosen to match with the five dimensional results. Closely related first order flow equations have recently been derived using similar methods in [22] for the $S T U$ model. ${ }^{14}$ In this case $\Omega_{a}$ reduces to three phases, which altogether with $\alpha$ define equivalently the first order equations. Nevertheless, the authors in [22] use the second order equations of motion to determine the evolution of these phases, whereas we consider the first order equations (3.19), which imply the second order ones by construction.

\footnotetext{
${ }^{14}$ I.e. for $\mathcal{M}_{4} \cong \mathrm{SU}(1,1) / \mathrm{U}(1) \times \mathrm{SU}(1,1) / \mathrm{U}(1) \times \mathrm{SU}(1,1) / \mathrm{U}(1)$.
} 
For the almost-BPS system, the eigenvalue equation (3.16) is defined by the generator $\mathbf{h}_{\mathrm{A}}=\mathbf{h}_{\mathrm{C}}+2 \mathbf{h}_{*}$, where we use the same generators as defined in (3.26) and (3.20) above. The corresponding graded decomposition of $\mathfrak{s l}_{2} \oplus \mathfrak{g}_{4}$ is then given by

$$
\mathfrak{s l}_{2} \oplus \mathfrak{g}_{4} \cong \mathbf{1}^{(-4)} \oplus\left(\mathbb{R}^{n_{v}}\right)^{(-2)} \oplus\left(\mathfrak{g l}_{1} \oplus \mathfrak{g l}_{1} \oplus \mathfrak{g}_{5}\right)^{(0)} \oplus\left(\overline{\mathbb{R}^{n_{v}}}\right)^{(2)} \oplus \mathbf{1}^{(4)},
$$

which is exactly the same as the one in (3.23) with respect to the $\mathfrak{g}_{4}$ components. The additional notation $\mathbb{R}^{n_{v}}$ and $\overline{\mathbb{R}^{n_{v}}}$ refers to two conjugate representations of the real group $\mathfrak{g}_{5}$. Similarly, the coset component is decomposed as

$$
\mathbf{2} \otimes \mathbb{R}^{2 n_{v}+2} \cong \mathbf{1}^{(-5)} \oplus\left(\overline{\mathbb{R}^{n_{v}}}\right)^{(-3)} \oplus\left(\mathbf{1} \oplus \mathbb{R}^{n_{v}}\right)^{(-1)} \oplus\left(\mathbf{1} \oplus \overline{\mathbb{R}^{n_{v}}}\right)^{(1)} \oplus\left(\mathbb{R}^{n_{v}}\right)^{(3)} \oplus \mathbf{1}^{(5)},
$$

so that $P \in\left(\mathbf{1} \oplus \overline{\mathbb{R}^{n_{v}}}\right)^{(1)} \oplus\left(\mathbb{R}^{n_{v}}\right)^{(3)} \oplus \mathbf{1}^{(5)}$, i.e. it lies in the positive grade component.

Due to the presence of the generator $\mathbf{h}_{*}$, which by itself defines the BPS system, ${ }^{15}$ the first order system reduces to the BPS system (3.21) for most of the components of the field strength $F$, except for the ones that define the solution

$$
Z=i e^{\frac{i}{2} \alpha} \mathcal{N}[\Omega]^{\frac{1}{2}} \mathrm{u}, \quad Z_{a}=i e^{\frac{i}{2} \alpha} \mathcal{N}[\bar{\Omega}]^{\frac{1}{2}} \Omega_{a} \mathrm{u},
$$

for which the expressions of $\mathrm{w}$ and $\Sigma^{a}$ take the opposite sign, where $\mathrm{u}$ is a real function. The projection to this component is obtained as

$$
\begin{aligned}
\mathcal{P} Z & =\frac{i}{4} e^{\frac{i \alpha}{2}} \mathcal{N}[\Omega]^{\frac{1}{2}} \operatorname{Im}\left[e^{-\frac{i \alpha}{2}} \mathcal{N}[\bar{\Omega}]^{\frac{1}{2}} Z+e^{-\frac{i \alpha}{2}} \mathcal{N}[\Omega]^{\frac{1}{2}} \bar{\Omega}^{a} Z_{a}\right] \\
\mathcal{P} Z_{a} & =\frac{i}{4} e^{\frac{i \alpha}{2}} \mathcal{N}[\bar{\Omega}]^{\frac{1}{2}} \Omega_{a} \operatorname{Im}\left[e^{-\frac{i \alpha}{2}} \mathcal{N}[\bar{\Omega}]^{\frac{1}{2}} Z+e^{-\frac{i \alpha}{2}} \mathcal{N}[\Omega]^{\frac{1}{2}} \bar{\Omega}^{b} Z_{b}\right]
\end{aligned}
$$

for which one easily computes that $\mathcal{P}^{2}=\mathcal{P}$. One can then verify that the first order system obtained by changing the sign of the components above, given by

$$
\begin{aligned}
\mathrm{w} & =e^{-i \alpha}(Z-2 \mathcal{P} Z) \\
& =\frac{3}{4} e^{-i \alpha} Z+\frac{1}{4} \mathcal{N}[\Omega] \bar{Z}+\frac{1}{4} \Omega_{a} \bar{Z}^{a}-\frac{1}{4} e^{-i \alpha} \mathcal{N}[\Omega] \bar{\Omega}^{a} Z_{a}, \\
\Sigma^{a} & =e^{i \alpha}\left(\bar{Z}^{a}-2 \mathcal{P} \bar{Z}^{a}\right) \\
& =e^{i \alpha} \bar{Z}^{a}+\frac{1}{4} \bar{\Omega}^{a}\left(Z+\mathcal{N}[\Omega] \bar{\Omega}^{b} Z_{b}-e^{i \alpha} \mathcal{N}[\Omega] \bar{Z}-e^{i \alpha} \Omega_{b} \bar{Z}^{b}\right),
\end{aligned}
$$

solves (3.16), as expected. Using the definitions in (3.9), these equations become a first order flow for the four dimensional scalars in terms of the gauge field strengths.

However, the auxiliary fields $e^{-i \alpha}$ and $\Omega_{a}$ parametrising $\mathbf{h}_{\mathrm{A}}$ are still arbitrary, we therefore need to impose (3.19) to obtain their dependence on the physical fields. First, the condition on $d_{B} \mathbf{h}_{*}$ to be of positive grade is the same as the one for the BPS system in (3.22) and leads to the condition, [34]

$$
d \alpha+Q+\frac{1}{2} e^{2 U} \star d \omega+2 \operatorname{Im}\left[e^{-i \alpha} Z\right]=0
$$

\footnotetext{
${ }^{15}$ Note that while the same generator appears in the reduction of the composite non-BPS system to the single centre class, we treat $\mathbf{h}_{\mathrm{A}}$ as the defining object here, instead of imposing a constraint similar to (2.59) on an existing system.
} 
where the last term is now given in terms of scalars by (3.39) and is therefore more complicated than in (3.22). Analysing the components of (3.19) along $\mathfrak{g}_{4}$ to obtain the remaining auxiliary components, $\Omega_{a}$, one finds that it reduces again to (3.29) in exactly the same way as for the corresponding quantities in the composite non-BPS system. In fact, one can easily check that $\gamma\left(d_{B} \mathbf{h}_{\mathrm{A}}\right)$ and $G^{a}{ }_{b}\left(d_{B} \mathbf{h}_{\mathrm{A}}\right)$ are exactly the same as in (3.30) since they only depend on the algebra (2.13). In contrast, manipulating the expression for $\Lambda_{a}\left(d_{B} \mathbf{h}_{\mathrm{A}}\right)$ is sensitive to the particular form for the scalar momenta in terms of the gauge fields. In order to write the resulting equation in a suggestive way, it is useful to draw intuition from the analogous vector in the single centre class, ${ }^{16}$ as well as from known multi-centre solutions, which indicate that the magnetic dual of the vector described by $\Omega_{a}$ is a more convenient variable. We therefore consider the dual very small vector of mass one, $R^{*}$, defined as in (2.49), i.e. we impose

$$
\Omega_{a}=e^{i \alpha} \bar{Z}\left(R^{*}\right) Z_{a}\left(R^{*}\right)
$$

With this definition, one can use (3.39) to show that

$$
\begin{aligned}
\Lambda_{a}\left(d_{B} \mathbf{h}_{\mathrm{A}}\right)= & e^{i \alpha}\left(\bar{Z}\left(d R^{*}\right) Z_{a}\left(R^{*}\right)+\bar{Z}\left(R^{*}\right) Z_{a}\left(d R^{*}\right)+2 \operatorname{Re}\left[e^{i \alpha} \Omega_{b} e_{i}^{b} d t^{i}\right] \bar{Z}\left(R^{*}\right) Z_{a}\left(R^{*}\right)\right) \\
& -\left(Z_{a}+\mathcal{N}[\Omega] c_{a b c} \bar{\Omega}^{b} \bar{Z}^{c}-\Omega_{a} \Omega_{b} \bar{Z}^{b}\right)
\end{aligned}
$$

As in (3.30), the second bracket in the last expression satisfies (3.29) identically, whereas the first term vanishes upon imposing that $R^{*}$ is related to a constant vector $\hat{R}^{*}$ by

$$
d R^{*}=-\operatorname{Re}\left[\bar{Z}\left(R^{*}\right) Z_{b}\left(R^{*}\right) e_{i}^{b} d t^{i}\right] R^{*}, \quad \Rightarrow \quad R^{*}=\frac{\hat{R}^{*}}{\left|Z\left(\hat{R}^{*}\right)\right|},
$$

similar to (3.32) for the composite system. In contrast to (3.30), there are two terms containing the derivative of $R^{*}$ in (3.42), so that one could expect more general solutions than the one shown above. However, one can verify that any solution for $R^{*}$ other than (3.43) is such that it is mutually nonlocal with its derivative $d R^{*}$, which is not allowed for an everywhere very small vector.

This concludes our presentation of the derivation of the first order flow equations for the almost-BPS system. One may now consider solutions to (3.39), which is a first order flow equation for the scalars $d U, \star d \omega$ and $d t^{i}$ in terms of the gauge fields and the constant vector $\hat{R}^{*}$, upon using (3.41) and (3.43). In section 5 we discuss the real form of these equations, show that they correspond to a linear system and give the characterisation of their solutions in terms of local functions.

\section{Composite non-BPS system}

In this section, we present in detail the steps required to characterise solutions of the flow equations for the composite non-BPS system in terms of local functions. The starting point

\footnotetext{
${ }^{16}$ Note that the non-BPS single centre system is also contained in the almost-BPS system, but we refrain from discussing the details of this reduction.
} 
is the solution of the nilpotency condition (3.27), written explicitly as a first order flow system for the four dimensional scalars and the metric degrees of freedom

$$
\begin{aligned}
d U+\frac{i}{2} e^{2 U} \star d \omega & =-\frac{1}{2} e^{U}\left(\Omega_{a} \bar{Z}^{a}(\star F)-\mathcal{N}[\Omega] \bar{Z}(\star F)\right) \\
-e_{i}^{a} d t^{i} & =c^{a b c} \Omega_{b} e^{U} Z_{c}(\star F)+\frac{1}{2} \bar{\Omega}^{a} e^{U}\left(Z(\star F)-\mathcal{N}[\Omega] \bar{\Omega}^{b} Z_{b}(\star F)\right),
\end{aligned}
$$

where $F$ is the spatial component of the field strengths defined in (3.3). The vector $\Omega_{a}$ is related to the constant very small vector, $\hat{R}$, through (3.28) and (3.32) above. For later reference, we give the inverse relations for the field strengths

$$
\begin{aligned}
e^{U} Z(\star F) & =\frac{1}{2} \mathcal{N}[\Omega]\left(d U-\frac{i}{2} e^{2 U} \star d \omega\right)-\frac{1}{2} \Omega_{b} e_{i}^{b} d t^{i} \\
e^{U} Z_{a}(\star F) & =-c_{a b c} \bar{\Omega}^{b} e_{i}^{c} d t^{i}+\frac{1}{2} \mathcal{N}[\bar{\Omega}] \Omega_{a} \Omega_{b} e_{i}^{b} d t^{i}-\frac{1}{2} \Omega_{a}\left(d U-\frac{i}{2} e^{2 U} \star d \omega\right) .
\end{aligned}
$$

In order to solve this system, we first construct the electromagnetic potentials and use the resulting structure to simplify the equations in section 4.1 . In section 4.2 we exhibit the relevance of the T-dualities introduced in section 2 . We then proceed to rewrite these equations as a linear system of differential equations and discuss its integration in terms of local functions in sections 4.3 and 4.4 respectively. The reader interested in applications can find a summary of the final form of the system in section 4.5.

\subsection{The electromagnetic potentials}

In order to solve the system (4.1), we construct the gauge field momenta (3.5) using the derivative of $R$ given by (3.32), to obtain

$$
\begin{aligned}
d \zeta & \equiv 2 e^{2 U} \operatorname{Re}\left[\bar{Z}(\star F) \mathcal{V}+\bar{Z}^{i}(\star F) D_{i} \mathcal{V}\right] \\
& =d\left(e^{U} \operatorname{Re}\left[\mathcal{N}[\bar{\Omega}] \mathcal{V}-\bar{\Omega}^{i} D_{i} \mathcal{V}\right]\right)+\frac{1}{2} e^{U} \operatorname{Im}\left[\mathcal{N}[\bar{\Omega}] \Omega_{i} d t^{i}\right] R+\frac{1}{4} e^{3 U} \star d \omega R,
\end{aligned}
$$

where we made extensive use of the special geometry identities in section A. The first term is manifestly a total derivative, whereas the others are along the very small vector $R$ and must therefore combine into the derivative of a single function. This requirement, along with (3.32), leads to the condition

$$
e^{U} \operatorname{Im}\left[\mathcal{N}[\bar{\Omega}] \Omega_{i} d t^{i}\right]+\frac{1}{2} e^{3 U} \star d \omega=M e^{3 U} \operatorname{Re}\left[\mathcal{N}[\bar{\Omega}] \Omega_{i} d t^{i}\right]-d\left(M e^{3 U}\right),
$$

where $M$ is an arbitrary function, so that the gauge field momenta take the form

$$
\zeta=e^{U} \operatorname{Re}\left[\mathcal{N}[\bar{\Omega}] \mathcal{V}-\bar{\Omega}^{i} D_{i} \mathcal{V}\right]-\frac{1}{2} e^{3 U} M R,
$$

with the corresponding central charges given by

$$
Z(\zeta)=\frac{i}{2} e^{U}\left(1+i e^{2 U} M\right) \mathcal{N}[\Omega], \quad Z_{a}(\zeta)=\frac{i}{2} e^{U}\left(1+i e^{2 U} M\right) \Omega_{a},
$$

for later reference. 
The structure of (4.5) can be used to show that one vector is always trivial, simplifying the system. To see that, we compute

$$
\langle\hat{R}, \zeta\rangle=-2 e^{U}|Z(\hat{R})|,
$$

whereas taking the imaginary part of (4.1) one gets

$$
e^{2 U} \star d \omega=-\frac{e^{U}}{2|Z(\hat{R})|}\langle\hat{R}, \star F\rangle .
$$

Finally, using (3.3), one finds that

$$
\langle\hat{R}, d w\rangle=0
$$

which implies that one vector field is always absent. In terms of the graded decomposition in section 2 , the vanishing component is along the very small vector dual to $R$, given in (2.49), as will be shown shortly.

One can now combine (4.6) and (4.9) to disentangle the term proportional to $\star d \omega$ in the definition of the scalar flow equation, so that (4.1) becomes

$$
\begin{aligned}
-e_{i}^{a} d t^{i}= & c^{a b c} \Omega_{b} e^{U} Z_{c}(\star d w)+\frac{1}{2} \bar{\Omega}^{a} e^{U}\left(Z(\star d w)-\mathcal{N}[\Omega] \bar{\Omega}^{b} Z_{b}(\star d w)\right) \\
& +\frac{1}{2} e^{4 U}\left(-M+i e^{-2 U}\right) \mathcal{N}[\Omega] \bar{\Omega}^{a} \star d \omega .
\end{aligned}
$$

Applying the same procedure on (4.2), one obtains the inverse relations of (4.10) for the central charges $Z(d w)$ and $Z_{a}(d w)$. The charge vectors $d w$ can then be straightforwardly constructed with the result

$$
\begin{gathered}
\star d w \equiv 2 \operatorname{Im}\left[-\bar{Z}(d w) \mathcal{V}+\bar{Z}^{a}(d w) D_{a} \mathcal{V}\right] \\
=e^{-U} \operatorname{Im}\left[-\left(d U+\frac{i}{2} e^{2 U} \star d \omega\right) \mathcal{R}_{+}+\left(i \bar{Y} e^{2 U} \star d \omega-\mathcal{N}[\Omega] \bar{\Omega}^{i} d \bar{t}_{i}\right) \mathcal{R}_{-}\right. \\
\left.\quad-2 c^{a b c} \Omega_{b} e_{c}^{i} d \bar{t}^{i} D_{a} \mathcal{V}+2 \mathcal{N}[\Omega] \bar{\Omega}^{i} d \bar{t}_{i} \bar{\Omega}^{j} D_{j} \mathcal{V}\right]
\end{gathered}
$$

where we used the shorthands

$$
\begin{aligned}
\mathcal{R}_{ \pm} & = \pm \mathcal{N}[\bar{\Omega}] \mathcal{V}+\bar{\Omega}^{i} D_{i} \mathcal{V} \\
Y & =\left(1+i e^{2 U} M\right)
\end{aligned}
$$

Note that the above results are in direct correspondence with the ones in [14], where the single centre system was treated.

\subsection{Connection to T-dualities}

Given the structure above, it is useful to define a second distinguished very small vector which is mutually nonlocal with $\hat{R}$, as in (2.49)-(2.50) through

$$
\hat{R}^{*}=|Z(\hat{R})|^{-1} \operatorname{Re}\left[\bar{Y}^{3} \mathcal{N}[\bar{\Omega}] \mathcal{V}+|Y|^{2} \bar{Y} \bar{\Omega}^{i} D_{i} \mathcal{V}\right] .
$$


Here, we identified the function $Y$ in (4.13) with the one in (2.50) and we included an overall rescaling. One can check that $\left\langle\hat{R}, \hat{R}^{*}\right\rangle=4$ and that the central charges of $\hat{R}^{*}$ above satisfy (2.18). This new vector is defined in exactly the same way as the second constant vector used in [14] in the single centre case, but is not constant in the full composite non-BPS system.

As explained in $(2.5)=(2.6)$, a non-constant very small vector with a constant non-zero symplectic product with $\hat{R}$ must be of the form

$$
\hat{R}^{*}=\exp \left[\mathrm{T}^{+}\right] R_{0}^{*} .
$$

where $R_{0}^{*}$ is a constant very small vector satisfying $\left\langle\hat{R}, R_{0}^{*}\right\rangle=4$ and $\exp \left[\mathrm{T}^{+}\right]$is a T-duality matrix leaving $\hat{R}$ invariant. It then follows that the derivative of $\hat{R}^{*}$ takes the form

$$
d \hat{R}^{*}-d \mathrm{~T}^{+} \hat{R}^{*}=0,
$$

which is identically closed by the fact that T-dualities are abelian. The above are consistent with known solutions $[12,13]$, in which $\hat{R}^{*}$ takes the form of a T-duality whose parameters are harmonic functions, acting on a constant vector along $p^{0}$.

Within the composite non-BPS system, one can explicitly compute the components of the derivative of $\hat{R}^{*}$ using (4.4) and (4.10), as

$$
\begin{gathered}
Z\left(d \hat{R}^{*}\right)=\mathrm{T}_{\gamma, Q}^{+} Z\left(\hat{R}^{*}\right)=Z\left(d \mathrm{~T}^{+} \hat{R}^{*}\right), \\
Z_{a}\left(d \hat{R}^{*}\right)=\mathrm{T}_{\gamma, Q}^{+} Z_{a}\left(\hat{R}^{*}\right)=Z_{a}\left(d \mathrm{~T}^{+} \hat{R}^{*}\right),
\end{gathered}
$$

where we indicated that the components in this basis are given by the variation of $\hat{R}^{*}$ itself under the T-duality transformations (2.56), as shown in (2.58). In order to obtain this result, one has to identify $\hat{R}$ as the grade $(+3)$ very small vector, that is invariant under all $\mathrm{T}$-dualities $\mathrm{T}^{+}$, which we assume henceforth. The explicit values for the parameters are given by

$$
\begin{aligned}
\gamma_{+} & =\frac{1}{6} e^{2 U}(d M-\star d \omega), \\
\bar{Q}_{+a} & =-\frac{1}{2}\left[Y \mathcal{N}[\Omega] N_{a}+\bar{Y} c_{a b c} \bar{\Omega}^{b} \bar{N}^{c}-\frac{1}{3} \Omega_{a}\left(2 \bar{Y} \mathcal{N}[\bar{\Omega}] \Omega_{b} \bar{N}^{b}+Y \mathcal{N}[\Omega] \bar{\Omega}^{b} N_{b}\right)\right],
\end{aligned}
$$

where we used the combination

$$
\bar{N}^{a}=-e_{i}^{a} d t^{i}+\mathcal{N}[\Omega] \bar{\Omega}^{a}\left(d U-\frac{i}{2} e^{2 U} \star d \omega\right),
$$

for brevity. Note that in the single centre class of [14] these expressions were shown to vanish, consistent with the fact that $\hat{R}^{*}$ is constant if the T-dualities are rigid.

Given the above, we can directly apply all considerations of section 2 , since the presence of the two constant very small vectors $\hat{R}$ and $R_{0}^{*}$ implies that the grading shown in (2.29) is relevant for the integration of the system. It follows that $R_{0}^{*}$ is identified as the grade $(-3)$ very small vector. As discussed in (2.58)-(2.59), a generic T-duality is parametrised by a rank three grade $(+1)$ vector, which we denote by $\mathcal{K}$, so that $(4.15)-(4.16)$ become

$$
\hat{R}^{*}=\exp \left[\mathrm{T}_{\mathcal{K}}^{+}\right] R_{0}^{*}, \quad d \mathrm{~T}_{\mathcal{K}}^{+} R_{0}^{*}=d \mathcal{K} .
$$


The rank of $\mathcal{K}$ can be verified by checking that the quartic invariant of the vector $d \hat{R}^{*}$ in (4.17) is vanishing, i.e.

$$
\begin{aligned}
I_{4}\left(d \hat{R}^{*}\right) & =I_{4}\left(d \mathrm{~T}_{\mathcal{K}}^{+} R_{0}^{*}\right)=I_{4}(d \mathcal{K})=0 \\
\partial_{\mu} \mathrm{T}_{\mathcal{K}}^{+} \partial_{\nu} \mathrm{T}_{\mathcal{K}}^{+} R_{0}^{*} & =-\frac{1}{16} I_{4}^{\prime}\left(\partial_{\mu} \mathrm{T}_{\mathcal{K}}^{+} R_{0}^{*}, \partial_{\nu} \mathrm{T}_{\mathcal{K}}^{+} R_{0}^{*}, \hat{R}\right)=-\frac{1}{16} I_{4}^{\prime}\left(\partial_{\mu} \mathcal{K}, \partial_{\nu} \mathcal{K}, \hat{R}\right) .
\end{aligned}
$$

In what follows, we will generally drop the subscript $\mathcal{K}$ on $\mathrm{T}_{\mathcal{K}}^{+}$for simplicity, since this is the only T-duality appearing throughout.

Finally, it is worth commenting on the difference between the dual very small vector in (2.49), which was used to define $\hat{R}^{*}$ in (4.14), and the constant vector $R_{0}^{*}$ of grade $(-3)$, that might seem confusing. It is important to realise that (2.49) simply defines a possible dual vector, which is not unique. Since two very small vectors not commuting with $\hat{R}$ are related by exactly a finite T-duality leaving $\hat{R}$ invariant, one may choose any other vector in that orbit. We will fix this ambiguity by defining the function $\mathcal{K}$ to vanish in the asymptotic region, or equivalently $R_{0}^{*}=\left.\hat{R}^{*}\right|_{r \rightarrow \infty}$.

\subsection{The linear system}

One can now use the vector $\hat{R}^{*}$ of (4.14) in a way similar to the vector $R$ was used in section 4.1, to project the flow equations and simplify the system. Indeed, taking the inner product with $d w$ one finds

$$
\left\langle\hat{R}^{*}, d w\right\rangle=-\star d\left(e^{-U}|Z(\hat{R})|^{-1}|Y|^{2}\right) \equiv-\star d V,
$$

where we defined the function $V$. Note that $V$ is not a harmonic function, since $\hat{R}^{*}$ is not a constant vector. Combining this with (3.32), (4.4) and (4.10), we can determine the combination $\Omega_{i} d t^{i}$ in terms of $V, M$ and the metric components as

$$
\begin{aligned}
\operatorname{Re}\left[\mathcal{N}[\bar{\Omega}] \Omega_{i} d t^{i}\right] & =|Z(\hat{R})|^{-1} d|Z(\hat{R})|=-V^{-1} d V-d U+|Y|^{-2} d|Y|^{2}, \\
\mathcal{N}[\bar{\Omega}] \Omega_{i} d t^{i} & =(1-2 Y) d U-\frac{1}{2} i e^{2 U} \star d \omega+\frac{Y^{2}}{|Y|^{2}} d \bar{Y}-V^{-1} Y d V .
\end{aligned}
$$

We now use all the above information to write the expression (4.11) for $d w$ in a suggestive form. To this end, we use the expression for the derivative of $\hat{R}^{*}$ in (4.17), combined with inspiration drawn from the analogous computation performed in [14] for the single centre class. After a long but straightforward computation we obtain

$$
\begin{aligned}
\star d w- & d\left(\frac{M}{V} \hat{R}^{*}\right)-\frac{1}{2} d V \hat{R}-2 e^{2 U} V d\left(\frac{M}{V}\right) \operatorname{Re}\left[e^{-U} e^{-i \alpha} \mathcal{V}\right] \\
= & 4 e^{-U} \operatorname{Im}\left[3 i \bar{Y} \mathcal{N}[\bar{\Omega}] \mathcal{V}-i(2 \bar{Y}-Y) \bar{\Omega}^{i} D_{i} \mathcal{V}\right] \gamma_{+} \\
& +e^{-U} \operatorname{Im}\left[\left(2 d U-i e^{2 U} \star d \omega\right) e^{-i \alpha} \mathcal{V}-2 e^{-i \alpha} d t^{i} D_{i} \mathcal{V}-4 Q_{+}^{a} D_{a} \mathcal{V}\right]
\end{aligned}
$$

Here, we used (2.50) to define a new phase as

$$
e^{-i \alpha} \equiv-\frac{\bar{Y}^{2}}{|Y|^{2}} \mathcal{N}[\bar{\Omega}]
$$


which will be useful in what follows. One can compute the derivative of $e^{-i \alpha}$ using (3.33) to first obtain

$$
\mathcal{N}[\Omega](d-i Q) \mathcal{N}[\bar{\Omega}]=\frac{i}{2} e^{2 U} \star d \omega+\frac{2}{|Y|^{2}} d Y-i e^{2 U} V d\left(\frac{M}{V}\right),
$$

from which follows the relation

$$
d \alpha+Q+\frac{1}{2} e^{2 U} \star d \omega-e^{2 U} d\left(\frac{M}{V}\right) V=0 .
$$

We emphasise that these equations are completely analogous to the ones relevant for the single centre system, but now involve generically non-harmonic functions $M$ and $V$.

Additionally, (4.25) contains the parameters for the particular T-duality appearing in the derivative of $\hat{R}^{*}$ in (4.18). These can be rewritten by observing that the action of a T-duality on the symplectic section takes the form

$$
\begin{aligned}
d \mathrm{~T}^{+} & {\left[2 e^{-U} \operatorname{Im}\left(e^{-i \alpha} \mathcal{V}\right)-\frac{M}{V} \hat{R}^{*}\right] } \\
& =2 e^{-U} \operatorname{Im}\left[-3 i \gamma_{*} Y e^{-i \alpha} \mathcal{V}+i \gamma_{*}(Y-2 \bar{Y}) \bar{\Omega}^{i} D_{i} \mathcal{V}-Q_{*}^{i} D_{i} \mathcal{V}\right]
\end{aligned}
$$

Using this relation and (4.28) in (4.25) leads to

$$
\star d w=-\left[d-2 d \mathrm{~T}^{+}\right]\left[2 \operatorname{Im}\left(e^{-U-i \alpha} \mathcal{V}\right)-\frac{1}{2} V \hat{R}-\frac{M}{V} \hat{R}^{*}\right],
$$

where we also used the fact that $\hat{R}$ is by definition inert under the T-dualities in question. This is the final form of the flow equations in the real basis, where the local T-dualities have parameters given by (4.18) above.

\subsection{Integration and local structure}

Due to the presence of the flat connection for the T-dualities, it is not possible to solve the system of equations (4.30) in terms of harmonic functions only. However, the scalar and vector fields can be written as

$$
\begin{aligned}
2 e^{-U} \operatorname{Im}\left(e^{-i \alpha} \mathcal{V}\right)-\frac{1}{2} V \hat{R}-\frac{M}{V} \hat{R}^{*} & =-\mathcal{H}, \\
d w & =\left(\star d-2 \star d \mathrm{~T}^{+}\right) \mathcal{H},
\end{aligned}
$$

where the vector of functions $\mathcal{H}$ is the solution to the non-harmonic equation

$$
d \star d \mathcal{H}-2 d \star d \mathrm{~T}^{+} \mathcal{H}-2 d \mathrm{~T}^{+} \wedge \star d \mathcal{H}=0 .
$$

In order to disentangle the derivatives on $\mathcal{H}$ and $\mathrm{T}^{+}$and cast this as a Poisson equation, we introduce the rescaled vector of functions

$$
\mathcal{H}_{0}=\exp \left[-\mathrm{T}^{+}\right] \mathcal{H}
$$

in terms of which we find

$$
2 e^{-U} \operatorname{Im}\left(e^{-i \alpha} \mathcal{V}\right)-\frac{1}{2} V \hat{R}-\frac{M}{V} \hat{R}^{*}=-\exp \left[\mathrm{T}^{+}\right] \mathcal{H}_{0},
$$




$$
d w=\exp \left[\mathrm{T}^{+}\right] \star d \mathcal{H}_{0}-\star d\left(\exp \left[\mathrm{T}^{+}\right]\right) \mathcal{H}_{0},
$$

while (4.33) takes the form

$$
d \star d \mathcal{H}_{0}-d \star d \mathrm{~T}^{+} \mathcal{H}_{0}-d \mathrm{~T}^{+} \wedge \star d \mathrm{~T}^{+} \mathcal{H}_{0}=0 .
$$

It is possible to give a systematic characterisation of the solution for $\mathcal{H}_{0}$ and $\exp \left[\mathrm{T}^{+}\right]$ using the following crucial observation. From the expression (4.36) for the vector fields, we compute for the derivative of $\mathcal{H}_{0}$ that

$$
\begin{aligned}
Z_{a}\left(\exp \left[\mathrm{T}^{+}\right] \star d \mathcal{H}_{0}\right) & -\mathcal{N}[\bar{\Omega}] \Omega_{a} Z\left(\exp \left[\mathrm{T}^{+}\right] \star d \mathcal{H}_{0}\right) \\
= & Z_{a}\left(\star d w+\exp \left[\mathrm{T}^{+}\right] d \mathrm{~T}^{+} \mathcal{H}_{0}\right)-\mathcal{N}[\bar{\Omega}] \Omega_{a} Z\left(\star d w+\exp \left[\mathrm{T}^{+}\right] d \mathrm{~T}^{+} \mathcal{H}_{0}\right) \\
= & -\frac{Y^{2}}{|Y|^{2}}\left(\bar{Q}_{+a}+2 i \Omega_{a} \gamma_{+}\right)+e^{i \alpha} N_{a}
\end{aligned}
$$

where $N_{a}$ is the combination of scalar momenta defined in (4.19). One can easily show that the central charges in (4.38) satisfy the reality constraint (2.59), i.e. we have

$$
\begin{aligned}
e^{i \alpha} & {\left[\bar{Z}^{a}\left(\exp \left[\mathrm{T}^{+}\right] d \mathcal{H}_{0}\right)-\mathcal{N}[\Omega] \bar{\Omega}^{a} \bar{Z}\left(\exp \left[\mathrm{T}^{+}\right] d \mathcal{H}_{0}\right)\right]=} \\
& =c^{a b c} \Omega_{b} Z_{c}\left(\exp \left[\mathrm{T}^{+}\right] d \mathcal{H}_{0}\right)+\bar{\Omega}^{a}\left(Z\left(\exp \left[\mathrm{T}^{+}\right] d \mathcal{H}_{0}\right)-\mathcal{N}[\Omega] \bar{\Omega}^{b} Z_{b}\left(\exp \left[\mathrm{T}^{+}\right] d \mathcal{H}_{0}\right)\right)
\end{aligned}
$$

This constraint was found to be crucial to describe single centre solutions in [14], where it was analysed in some detail. Using (2.30), one computes that it is equivalent to the constraints

$$
\begin{aligned}
\frac{1}{2} I_{4}^{\prime}\left(\hat{R}, \hat{R}^{*}, \exp \left[\mathrm{T}^{+}\right] d \mathcal{H}_{0}\right) & =-4 \exp \left[\mathrm{T}^{+}\right] d \mathcal{H}_{0}+3\left\langle\exp \left[\mathrm{T}^{+}\right] d \mathcal{H}_{0}, \hat{R}^{*}\right\rangle \hat{R} \\
\left\langle\hat{R}, \exp \left[\mathrm{T}^{+}\right] d \mathcal{H}_{0}\right\rangle & =0
\end{aligned}
$$

These equations are manifestly duality covariant, and in particular T-duality covariant. Therefore, using (4.15) one finds that the vector $d \mathcal{H}_{0}$ satisfies the constraints

$$
\frac{1}{2} I_{4}^{\prime}\left(\hat{R}, R_{0}^{*}, d \mathcal{H}_{0}\right)=-4 d \mathcal{H}_{0}+3\left\langle d \mathcal{H}_{0}, R_{0}^{*}\right\rangle \hat{R}, \quad\left\langle\hat{R}, d \mathcal{H}_{0}\right\rangle=0
$$

In a similar fashion, one can show that the vector of functions $\mathcal{H}_{0}$ itself satisfies the same constraint, using (4.31), and therefore only half of its components are allowed.

Indeed, a vector satisfying this constraint does not contain components of grade $(+1)$ and $(-3)$, so that in terms of $(2.29)$, one finds

$$
\mathcal{H}_{0} \in\left(\mathbb{R}^{n_{v}}\right)^{(-1)} \oplus \mathbb{R}^{(3)}
$$

and therefore describes only $n_{v}+1$ functions instead of the $2\left(n_{v}+1\right)$ one would have a priori, i.e. it lies on a Lagrangian subspace. One of these functions is clearly the function $V$ in (4.23), describing the grade $(+3)$ component of $\mathcal{H}_{0}$ along the direction of $R$. The remaining $n_{v}$ functions span the grade $(-1)$ vector space, according to the decomposition imposed by the T-duality $\mathrm{T}^{+}$, and are undetermined for the moment. 
Using this decomposition in (4.37), we find the following grade assignments for each term

$$
\begin{aligned}
& d \star d \mathcal{H}_{0}-d \star d \mathrm{~T}^{+} \mathcal{H}_{0}-d \mathrm{~T}^{+} \wedge \star d \mathrm{~T}^{+} \mathcal{H}_{0}=0, \\
& (-1) \oplus(+3)
\end{aligned}
$$

where we used the fact that $\mathrm{T}^{+}$is of grade $(+2)$. Now, since the second term in (4.43) lies in a subspace orthogonal to both the other terms, it is clear that this equation decomposes in two independent equations, as

$$
\begin{aligned}
d \star d \mathcal{H}_{0}-d \mathrm{~T}^{+} \wedge \star d \mathrm{~T}^{+} \mathcal{H}_{0} & =0, \\
d \star d \mathrm{~T}^{+} & =0 .
\end{aligned}
$$

In deriving the second equation we used the fact that applying a T-duality on a generic vector as in (4.42) results in a vector of grade $(+1)$, which cannot vanish for a physical solution ${ }^{17}$ unless the T-duality matrix is trivial. We now analyse each of the two equations in turn.

First, it is easy to express (4.45) in terms of the vector of T-duality parameters in (4.20), as

$$
d \star d \mathcal{K}=0,
$$

so that the parameters $\mathcal{K}$ are identified as a rank three vector of harmonic functions. Note that $\mathcal{K}$ is a priori a generic vector of grade $(-1)$, i.e. it lies in the same Lagrangian subspace as the vector $\mathcal{H}_{0}$ above, with the additional restriction of a vanishing component along $R$, since $\left\langle\hat{R}^{*}, d \hat{R}^{*}\right\rangle=0$ by definition. However, we should note that the constant part of $\mathcal{K}$ is not physical and can be absorbed into $R_{0}^{*}$ by imposing the boundary condition that $\mathcal{K}$ vanishes asymptotically. This choice is useful in the discussion of explicit solutions.

We now turn to the Poisson equation (4.44) for the vector $\mathcal{H}_{0}$. As shown in (4.43), the source term of is along the unique grade $(+3)$ component, that is along the vector $R$. We have therefore identified $\mathcal{H}_{0}$ as a vector lying in a Lagrangian submanifold containing $R$, whose components along the grade $(-1)$ directions are $n_{v}$ harmonic functions and the component along the direction of $R$ is a single non-harmonic function, $V$. One can directly compute the source term by varying the combination in (4.29) and using (4.35), to find

$$
\begin{aligned}
d \mathrm{~T}^{+} \wedge \star d \mathrm{~T}^{+} \mathcal{H}_{0} & =-d \mathrm{~T}^{+} \wedge \star d \mathrm{~T}^{+}\left[2 \operatorname{Im}\left(e^{-i \alpha} \mathcal{V}\right)-\frac{M}{V} \hat{R}^{*}\right] \\
& =\left(6 \gamma_{+} \wedge \star \gamma_{+}-Q_{+}^{a} \wedge \star \bar{Q}_{+a}\right) R,
\end{aligned}
$$

which is explicitly proportional to the vector $R$, up to a real function. We can write this result in a simpler form by taking the inner product of (4.36) with $\hat{R}^{*}$ and comparing with (4.23), to obtain

$$
V=-\left\langle\hat{R}^{*}, \mathcal{H}\right\rangle=-\left\langle R_{0}^{*}, \mathcal{H}_{0}\right\rangle
$$

\footnotetext{
${ }^{17}$ The non-generic solutions to this equation require constraints on the vector of T-duality parameters and only exist if $I_{4}\left(\mathcal{H}_{0}, \mathcal{H}_{0}, \mathcal{H}_{0}, \hat{R}\right)=0$, which would lead to solutions with irregular horizons.
} 
where we used (4.15) and (4.34). The Poisson equation for this function can now be found by projecting (4.44) along $R_{0}^{*}$, as

$$
d \star d V=-\left\langle R_{0}^{*}, d \mathrm{~T}^{+} \wedge \star d \mathrm{~T}^{+} \mathcal{H}_{0}\right\rangle=-\frac{1}{16} I_{4}\left(d \mathcal{K}, \star d \mathcal{K}, \mathcal{H}_{0}, \hat{R}\right) .
$$

Note that, by (4.47), the right hand side of the first equality is nonvanishing, since it is the inner product of $R$ with its magnetic dual. The second equality is a direct consequence of (2.40). We moreover record the following relations for the expressions involving T-duality matrices in (4.29) and (4.47),

$$
\begin{aligned}
d \mathrm{~T}^{+}\left[2 e^{-U} \operatorname{Im}\left(e^{-i \alpha} \mathcal{V}\right)-\frac{M}{V} \hat{R}^{*}\right] & =\frac{1}{16} \exp \left(\mathrm{T}^{+}\right) I_{4}^{\prime}\left(d \mathcal{K}, \mathcal{H}_{0}, \hat{R}\right), \\
d \mathrm{~T}^{+} \wedge \star d \mathrm{~T}^{+}\left[2 \operatorname{Im}\left(e^{-i \alpha} \mathcal{V}\right)-\frac{M}{V} \hat{R}^{*}\right] & =\frac{1}{64} I_{4}\left(d \mathcal{K}, \star d \mathcal{K}, \mathcal{H}_{0}, \hat{R}\right) \hat{R}
\end{aligned}
$$

which are direct consequences of (2.35) and (2.40), in combination with (4.35). These equations, along with (4.22), allow us to evaluate the action of T-duality generators on the various objects relevant to the system. As the action of a finite T-duality is expressed as a finite sum due to the nilpotency of $\mathrm{T}^{+}$, it follows that one may also compute the action of finite T-dualities, as alluded to above. This is especially important when trying to find explicit solutions to this system [27].

The final quantities to be fixed are the angular momentum vector, $\omega$, and the function, $M$, appearing in the expression for the scalars. In the single centre class, these are both harmonic and are dual to each other. Indeed, in that case the T-duality parameters in (4.18) must vanish and the first of these equations imposes exactly that $M$ and $\omega$ are harmonic. In the more general multi-centre case, one has to compute the nontrivial $\gamma_{+}$to obtain the analogous equation. This can be done straightforwardly using (4.18), (4.34)(4.36) and (4.50) to show that

$$
\star d \omega-d M=\langle\mathcal{H}, \star d w\rangle=\left\langle\mathcal{H}_{0}, d \mathcal{H}_{0}-d \mathrm{~T}^{+} \mathcal{H}_{0}\right\rangle=\frac{1}{16} I_{4}\left(d \mathcal{K}, \mathcal{H}_{0}, \mathcal{H}_{0}, \hat{R}\right) .
$$

One can alternatively obtain the same expression by manipulating the definition of the non-harmonic function $M$ in (4.4) using the above results. The integrability condition on (4.51) leads to the following Poisson equation for $M$ :

$$
d \star d M=-2\left\langle\mathcal{H}_{0}, d \mathrm{~T}^{+} \star d \mathcal{H}_{0}\right\rangle=\frac{1}{8} I_{4}\left(d \mathcal{K}, \star d \mathcal{H}_{0}, \mathcal{H}_{0}, \hat{R}\right),
$$

which can be solved once the grade -1 component of $\mathcal{H}_{0}$ and $\mathcal{K}$ are chosen.

This concludes our duality covariant presentation of the composite non-BPS system in terms of the real basis. In the next section, we summarise the final form of the equations to be solved and we comment on some properties of the solutions.

\subsection{Summary of results}

In this short section, we summarise all relevant formulae for the composite non-BPS system in the real basis. All relations presented here were shown explicitly in the previous sections 
and we refer to the discussion there for the details. We find it however useful for future applications to give a self-contained account of the final form of the system.

The ansatze for the metric and gauge fields are given in (3.1) and (3.3) in terms of the function $e^{U}$, the one-form $\omega$ and the spatial vector fields $d w$, while the electromagnetic potentials are fixed by (4.5). The first order flow equation for the composite non-BPS system is given by (4.30), as

$$
\star d w=-\left[d-2 d \mathrm{~T}_{\mathcal{K}}^{+}\right]\left[2 \operatorname{Im}\left(e^{-U-i \alpha} \mathcal{V}\right)-\frac{1}{2} V \hat{R}-\frac{M}{V} \hat{R}^{*}\right] .
$$

Here, $M, V$, are functions to be specified below, while $\hat{R}$ and $\hat{R}^{*}$ are a constant and a non-constant very small vector respectively, where $\left\langle\hat{R}, \hat{R}^{*}\right\rangle=4$. The non-constant $\hat{R}^{*}$ is related to a constant very small vector, $R_{0}^{*}$, by $(4.15)$

$$
\hat{R}^{*}=\exp \left[\mathrm{T}_{\mathcal{K}}^{+}\right] R_{0}^{*}
$$

which also satisfies $\left\langle\hat{R}, R_{0}^{*}\right\rangle=4$. In this and all equations in this section, $\mathrm{T}_{\mathcal{K}}^{+}$is a generator of the T-dualities leaving $\hat{R}$ invariant, parametrised by a vector of harmonic functions, $\mathcal{K}$. As discussed in section 2 , the vector of parameters $\mathcal{K}$ lies in the grade $(-1)$ component of the vector space according to the decomposition implied by the T-duality. It is therefore a three-charge vector satisfying

$$
\frac{1}{2} I_{4}^{\prime}\left(\hat{R}, R_{0}^{*}, \mathcal{K}\right)=-\left\langle\hat{R}, R_{0}^{*}\right\rangle \mathcal{K}
$$

which indeed specifies a vector of $n_{v}$ degrees of freedom.

The solutions to the flow equation (4.53) are simplified by introducing a vector, $\mathcal{H}_{0}$, of grade $(-1) \oplus(+3)$, i.e. satisfying

$$
\frac{1}{2} I_{4}^{\prime}\left(\hat{R}, R_{0}^{*}, \mathcal{H}_{0}\right)=-\left\langle\hat{R}, R_{0}^{*}\right\rangle \mathcal{H}_{0}+3\left\langle\mathcal{H}_{0}, R_{0}^{*}\right\rangle \hat{R} .
$$

Note that (4.55) is trivially a solution of the first equation, found by setting the grade $(+3)$ component, $\left\langle R_{0}^{*}, \mathcal{H}_{0}\right\rangle$, to vanish. The equations resulting from (4.53) take the form (4.36),

$$
\begin{aligned}
2 e^{-U} \operatorname{Im}\left(e^{-i \alpha} \mathcal{V}\right) & =-\exp \left[\mathrm{T}_{\mathcal{K}}^{+}\right]\left(\mathcal{H}_{0}-\frac{1}{2} V \hat{R}-\frac{M}{V} R_{0}^{*}\right), \\
\star d w & =\exp \left[\mathrm{T}_{\mathcal{K}}^{+}\right]\left(d \mathcal{H}_{0}-d \mathrm{~T}_{\mathcal{K}}^{+} \mathcal{H}_{0}\right),
\end{aligned}
$$

where $V$ is now identified with the grade $(+3)$ component of $\mathcal{H}_{0}$, as $V=\left\langle\mathcal{H}_{0}, R_{0}^{*}\right\rangle$. The compatibility relation for the last relations leads to the field equation for $\mathcal{H}_{0}$, as in (4.44) and (4.50)

$$
d \star d \mathcal{H}_{0}=d \mathrm{~T}_{\mathcal{K}}^{+} \wedge \star d \mathrm{~T}_{\mathcal{K}}^{+} \mathcal{H}_{0}=-\frac{1}{64} I_{4}\left(d \mathcal{K}, \star d \mathcal{K}, \mathcal{H}_{0}, \hat{R}\right) \hat{R} .
$$

As the right hand side of this relation is only along $\hat{R}$, it follows that all grade $(-1)$ components of $\mathcal{H}_{0}$ are harmonic, whereas $V$ is not, leading to (4.49), as

$$
d \star d V=-\frac{1}{16} I_{4}\left(d \mathcal{K}, \star d \mathcal{K}, \mathcal{H}_{0}, \hat{R}\right)
$$


by taking the inner product of (4.59) with $R_{0}^{*}$. The final dynamical equation required is the one for the function $M$ in (4.58) and the angular momentum vector $\omega$, both of which are conveniently given by (4.51), as

$$
\star d \omega-d M=\left\langle\mathcal{H}_{0}, d \mathcal{H}_{0}-d \mathrm{~T}_{\mathcal{K}}^{+} \mathcal{H}_{0}\right\rangle=\frac{1}{16} I_{4}\left(d \mathcal{K}, \mathcal{H}_{0}, \mathcal{H}_{0}, \hat{R}\right) .
$$

Taking the divergence of this equation, one obtains a Poisson equation for $M$.

These equations can be seen to be equivalent to the known formulation of the composite non-BPS system, as given in a fixed duality frame in $[12,13]$, by making a choice for the constant vectors $\hat{R}$ and $R_{0}^{*}$. In fact, these two papers use different frames for describing the system, both of which can be reached from our general formulation. The frame of [13] is found by choosing

$$
\hat{R} \propto\left(0, \delta_{I}^{0}\right), \quad R_{0}^{*} \propto\left(\delta_{0}^{I}, 0\right),
$$

where we disregard the (arbitrary) normalisation. Similarly the frame in [12] is found by interchanging the expressions for the two vectors in (4.62), corresponding to an electric/magnetic duality.

We close with some comments on the structure of the solutions. First, the physical scalars and the metric scale factor can be obtained by solving (4.57) in the standard way [8]. Since all quantities above are appropriate combinations of the single centre solution in [14], up to overall T-dualities, it is possible to use many of the results given there. For instance, the metric scale factor is given by

$$
e^{-4 U}=I_{4}\left(\mathcal{H}_{0}-\frac{1}{2} V \hat{R}-\frac{M}{V} R_{0}^{*}\right)
$$

where we used the fact that the quartic invariant is by definition invariant under all Tdualities. This expression is identical to the corresponding one for the single centre class, despite the fact that the functions $M$ and $V$ are not harmonic in the present context. As in [14], one can simplify the expression for $e^{U}$ as follows. The decomposition of the vector $\mathcal{H}_{0}-\frac{1}{2} V \hat{R}$ in grades $(-1) \oplus(+3)$ implies that $I_{4}\left(\mathcal{H}_{0}-\frac{1}{2} V \hat{R}\right)$ is linear in $V$, as any other power of the grade $(+3)$ would vanish identically. In particular we find that

$$
\mathcal{H}_{0}-\frac{1}{4} V \hat{R} \in\left(\mathbb{R}^{n_{v}}\right)^{(-1)}, \quad \Rightarrow \quad I_{4}\left(\mathcal{H}_{0}-\frac{1}{4} V \hat{R}\right)=0,
$$

so that we can show the equality $I_{4}\left(\mathcal{H}_{0}-\frac{1}{2} V \hat{R}\right)=-I_{4}\left(\mathcal{H}_{0}\right)$. It then follows that

$$
\begin{aligned}
e^{-4 U} & =I_{4}\left(\mathcal{H}_{0}-\frac{1}{2} V \hat{R}\right)-\frac{M^{2}}{V^{2}}\left\langle R_{0}^{*}, \mathcal{H}_{0}-\frac{1}{2} V \hat{R}\right\rangle^{2} \\
& =-I_{4}\left(\mathcal{H}_{0}\right)-M^{2} .
\end{aligned}
$$

This equation implies that the $n_{v}$ grade $(-1)$ harmonic components in $\mathcal{H}_{0}$ must correspond to a rank three charge (i.e. a large electric charge in five dimensions), so that (4.65) leads to a non-degenerate metric.

Based on (4.65), we conclude that the system above indeed describes the interactions of black holes that are non-supersymmetric in isolation, since $I_{4}\left(\mathcal{H}_{0}\right)$ must be negative 
globally and in particular at each centre, for a regular geometry. We refrain from giving an explicit expression for the physical scalars, as these would involve the action of an arbitrary abelian isometry with parameters $\mathcal{K}$ on the physical scalars for the single centre class as given explicitly in [14]. Of course, the scalars can be computed from the standard formulae in [8] for any desired solution.

The characteristic features of all solutions in this class is that each one of the centres must be of the non-BPS type in isolation, as explained above, and that the charges at all centres must commute with the vector $\hat{R}$ and must not commute with the vector $R_{0}^{*}$, by regularity. It is then clear that such solutions do not exist for generic non-BPS charges at the centres. In addition, once an allowed charge configuration is fixed, one cannot have arbitrary values for the moduli at infinity. This is due to the constraint on $\mathcal{H}_{0}$, which contains only $n_{v}+1$ asymptotic constants and the fact that some of these constants, together with the $2 n_{v}$ parameters in $\hat{R}$ and $R_{0}^{*}$, turn out to parametrise charges that are not described by the poles of $\mathcal{H}_{0}$, through (4.53) and (4.58). A simple example of this situation is given by the class of two centre solutions for models with $n_{v} \geq 3$. In this case, the two non-BPS charges are in fact arbitrary, since one can always find a choice of $\hat{R}$ and $R_{0}^{*}$ for any pair of non-BPS charges. However, it is possible to show [27] that the asymptotic moduli are constrained to lie on a $\left(n_{v}+2\right)$-dimensional hypersurface of the $2 n_{v}$-dimensional moduli space. Note that this is very different from the multi-centre BPS solutions, where solutions exist a priori everywhere in moduli space and walls of marginal stability arise only when the constrains implied by global regularity are imposed. In the present case however, local solutions seem to exist only in certain hypersurfaces of moduli space, while walls of marginal stability might still arise on these constrained surfaces.

Finally, it is worthwhile commenting on the evaluation of T-dualities appearing as matrices in the equations above. As we have shown in several examples, one can avoid introducing explicit matrices, instead computing the action of T-dualities on the relevant vectors by use of the definition (4.46) and equations (4.50). Indeed, we find that it is possible to reduce all required computations to a recursive application of these three relations. As the grading involves only four subspaces, this procedure terminates after at most three steps.

\section{Almost-BPS system}

In this section we present in detail the characterisation of solutions to the almost-BPS system of equations, in analogy with the steps taken in the previous section for the composite non-BPS system. While the discussion here is self-contained, we will occasionally refer to section 4, in order to highlight similarities and recycle some results. The starting point is the solution to the nilpotency condition given in (3.39), which we repeat explicitly here

$$
\begin{aligned}
d U+\frac{i}{2} e^{2 U} \star d \omega & =-\frac{3}{4} e^{-i \alpha} Z-\frac{1}{4} \mathcal{N}[\Omega] \bar{Z}-\frac{1}{4} \Omega_{a} \bar{Z}^{a}+\frac{1}{4} e^{-i \alpha} \mathcal{N}[\Omega] \bar{\Omega}^{a} Z_{a} \\
-e_{i}^{a} d t^{i} & =e^{i \alpha} \bar{Z}^{a}+\frac{1}{4} \bar{\Omega}^{a}\left(Z+\mathcal{N}[\Omega] \bar{\Omega}^{b} Z_{b}-e^{i \alpha} \mathcal{N}[\Omega] \bar{Z}-e^{i \alpha} \Omega_{b} \bar{Z}^{b}\right)
\end{aligned}
$$

where $Z=Z(\star F), Z_{a}=Z_{a}(\star F)$ are the central charges of the spatial field strengths $F$ in (3.3). The vector $\Omega_{a}$ is connected to a constant very small vector, $\hat{R}^{*}$, through (3.41)- 
(3.43). For later reference, we also give the inverse relations, which read

$$
\begin{aligned}
Z & =-e^{i \alpha}\left(d U+\frac{i}{2} e^{2 U} \star d \omega\right)+\frac{i}{2} e^{\frac{i \alpha}{2}} \mathcal{N}[\Omega]^{\frac{1}{2}} \mu \\
Z_{a} & =-e^{i \alpha} e_{a i} d \bar{t}^{i}+\frac{i}{2} e^{\frac{i \alpha}{2}} \mathcal{N}[\bar{\Omega}]^{\frac{1}{2}} \Omega_{a} \mu
\end{aligned}
$$

where we used as shorthand the one-form

$$
\mu=\operatorname{Im}\left[e^{\frac{i \alpha}{2}} \mathcal{N}[\bar{\Omega}]^{\frac{1}{2}}\left(d U+\frac{i}{2} e^{2 U} \star d \omega\right)+e^{\frac{i \alpha}{2}} \mathcal{N}[\Omega]^{\frac{1}{2}} \bar{\Omega}^{a} e_{a i} d \bar{t}^{i}\right] .
$$

The final required equation is the compatibility equation (3.40), which can be rearranged using (5.2) and (5.3) to obtain

$$
d(\alpha-\arg [\mathcal{N}[\Omega]])=-\frac{2}{|Y|^{2}} \operatorname{Im}\left[Y\left(d U+\frac{i}{2} e^{2 U} \star d \omega-e^{-i \alpha} \Omega^{a} e_{i}^{a} d t^{i}\right)\right] .
$$

Here, we used the function $Y$ defined in the first equality of (2.50) and the fact that it has unit real part. Similarly, one can show using the flow equations above, that $\mu$ is also given by the expressions

$$
\begin{aligned}
\operatorname{Re}\left(e^{\left.\frac{-i \alpha}{2} \mathcal{N}[\Omega]^{\frac{1}{2}}\right) \mu}\right. & =\left(e^{2 U} \star d \omega+2 \operatorname{Im}\left[e^{-i \alpha} Z\right]\right) \\
& =\frac{1}{2} e^{2 U} d \omega-\operatorname{Im}\left(e^{-i \alpha} \Omega_{i} d t^{i}\right)+i|Y|^{-2} d \bar{Y}
\end{aligned}
$$

that will be used in due time.

In order to solve these equations, we follow a path similar to the last section, by considering the electromagnetic potentials in section 5.1 and using them to simplify the equations. The connection with T-dualities is shown in section 5.2, while sections 5.3 and 5.4 are devoted to the linear system of equations governing this system and its integration in terms of local functions respectively. The reader interested in applications can find a summary of the final form of the system in section 4.5.

\subsection{The electromagnetic potentials}

As a first step towards the solution of the system, we decompose the field strength $F$ in the electromagnetic potentials and the vector fields that define the conserved charges. The electromagnetic potentials for this system are computed by their definition (3.5) as

$$
\begin{aligned}
d \zeta & \equiv 2 e^{2 U} \operatorname{Re}\left[\bar{Z}(\star F) \mathcal{V}+\bar{Z}^{i}(\star F) D_{i} \mathcal{V}\right] \\
& =-2 d\left(e^{U} \operatorname{Re}\left[e^{-i \alpha} \mathcal{V}\right]\right)+\frac{1}{2} e^{U} \mu R^{*},
\end{aligned}
$$

where we used (5.4) and the definition (3.41) to rearrange terms. The first term in (5.6) is already a total derivative, so that the last term must combine into the derivative a vector, which is necessarily proportional to $R^{*}$. Using the relation of $R^{*}$ to a constant vector in (3.43), this requirement leads to the condition

$$
e^{U} \mu=-2 W \operatorname{Re}\left[\bar{Z}\left(R^{*}\right) Z_{b}\left(R^{*}\right) e_{i}^{b} d t^{i}\right]+2 d W,
$$


where we introduced the a priori arbitrary real function $W$. The result for the electromagnetic potentials takes the form

$$
\zeta=-2 e^{U} \operatorname{Re}\left[e^{-i \alpha} \mathcal{V}\right]+W R^{*},
$$

while the corresponding central charges are

$$
\begin{aligned}
Z(\zeta) & =-i e^{U} e^{i \alpha}+W Z\left(R^{*}\right)=-i e^{i \alpha}\left(e^{U}+i W e^{\frac{i \alpha}{2}} \mathcal{N}[\bar{\Omega}]^{\frac{1}{2}}\right) \\
Z_{a}(\zeta) & =W Z_{a}\left(R^{*}\right)=W e^{\frac{i \alpha}{2}} \mathcal{N}[\bar{\Omega}]^{\frac{1}{2}} \Omega_{a} .
\end{aligned}
$$

We can now construct the vector potentials by the definition

$$
Z(d w)=Z(F)-Z(\zeta) d \omega, \quad Z_{a}(d w)=Z_{a}(F)-Z_{a}(\zeta) d \omega,
$$

which leads to the expression

$$
\star d w=2 \operatorname{Im}\left[-d\left(e^{-U} e^{-i \alpha} \mathcal{V}\right)+\left(\frac{i}{2} e^{-U} \mu+W d \omega\right) \mathcal{R}^{*}+i e^{-U} \mu e^{-\frac{i \alpha}{2}} \mathcal{N}[\bar{\Omega}]^{\frac{1}{2}} \mathcal{V}\right],
$$

where we use the shorthand in (5.3) and

$$
\mathcal{R}^{*}=e^{-\frac{3 i \alpha}{2}} \mathcal{N}[\Omega]^{\frac{1}{2}} \mathcal{V}-e^{-\frac{i \alpha}{2}} \mathcal{N}[\Omega]^{\frac{1}{2}} \bar{\Omega}^{a} D_{a} \mathcal{V} .
$$

Here, it is worth pointing out that, unlike in the composite non-BPS system (cf. (4.9)), there is no component of the vector fields that is vanishing a priori. Nevertheless, the projection of the vector fields in (5.11) along the available constant vector $\hat{R}^{*}$ is still relevant and can be computed as

$$
\begin{aligned}
\left\langle\hat{R}^{*}, d w\right\rangle= & 2\left(1+4 e^{-U} \frac{W}{|Y|}\right) \star d\left(e^{-U} \frac{\left|Z\left(\hat{R}^{*}\right)\right|}{|Y|}\right) \\
& -8 e^{-U} \frac{\left|Z\left(\hat{R}^{*}\right)\right|}{|Y|} \star d\left(e^{-U} \frac{W}{|Y|}\right),
\end{aligned}
$$

where $Y$ is again as in (2.50). This can be simplified by imposing consistency of the two expressions for $\mu$ in (5.5) with (5.7), combined with (3.43), leading to

$$
\left(1+2 e^{-U} \frac{W}{|Y|}\right) d\left(e^{-U} \frac{\left|Z\left(\hat{R}^{*}\right)\right|}{|Y|}\right)=2 e^{-U} \frac{\left|Z\left(\hat{R}^{*}\right)\right|}{|Y|} d\left(e^{-U} \frac{W}{|Y|}\right) .
$$

Now, it is simple to show that (5.13) and (5.14) imply that the projection $\left\langle\hat{R}^{*}, d w\right\rangle$ is given by a harmonic function, $V$, defined as

$$
\left\langle\hat{R}^{*}, d w\right\rangle=-2 \star d\left(e^{-U} \frac{\left|Z\left(\hat{R}^{*}\right)\right|}{|Y|}\right) \equiv-\star d V,
$$

while the function $W$ is fixed as

$$
1+2 e^{-U} \frac{W}{|Y|}=\beta V
$$

where $\beta$ is an arbitrary constant. Using these results, (5.7) simplifies to

$$
\mu=|Y| \frac{d V}{V},
$$

which will be used in due time. 


\subsection{Connection to T-dualities}

We now discuss the relevance of T-dualities for the almost-BPS system, which will be important for the integration of the flow equations, as for the composite non-BPS system in the previous section. In order to exhibit this, we consider the very small vector

$$
R=\operatorname{Im}\left[-\mathcal{N}[\bar{\Omega}] \mathcal{V}+\bar{\Omega}^{a} D_{a} \mathcal{V}\right]
$$

which is always mutually nonlocal with the constant vector $\hat{R}^{*}$ and is not constant in general. As is clear by their definitions these two vectors are related in exactly the same way as the pair of very small vectors, $R$ and $R^{*}$ in (2.17) and (2.49) respectively, up to rescalings. Note that the situation is opposite to the one for the composite non-BPS system, where $R$ is a constant vector up to rescaling, while $\hat{R}^{*}$ is not constant.

Based on the discussion in section 2, we associate $R$ to the grade $(+3)$ component of the decomposition (2.29), while $R^{*}$ is identified as the corresponding grade (-3) component. Moreover, one can check that the normalised very small vector

$$
\hat{R}=2\left|Z\left(\hat{R}^{*}\right)\right|^{-1}|Y|^{3} \operatorname{Im}\left[-\mathcal{N}[\bar{\Omega}] \mathcal{V}+\bar{\Omega}^{a} D_{a} \mathcal{V}\right],
$$

has a constant inner product with $\hat{R}^{*}$, namely $\left\langle\hat{R}, \hat{R}^{*}\right\rangle=4$. As explained in (2.5)-(2.6), the condition that $\hat{R}$ is a very small vector can be generally written as

$$
\hat{R}=\exp \left[\mathrm{T}^{-}\right] R_{0},
$$

where $R_{0}$ is a constant very small vector and $\exp \left[\mathrm{T}^{-}\right]$is a T-duality matrix leaving $\hat{R}^{*}$ invariant, parametrised by a grade $(+1)$ vector of functions. It follows that the derivative of $\hat{R}$ can be expressed as

$$
d \hat{R}-d \mathrm{~T}^{-} \hat{R}=0,
$$

which is closed by the property that T-dualities are abelian, exactly as in the composite non-BPS case. This is consistent with the known formulation of the almost-BPS system in five dimensions [15-17], which can be written in terms of a T-duality parametrised by harmonic functions, acting on the scalar and vector fields.

We can find the relevant T-duality parameters in (5.20) by explicitly computing the derivative of $\hat{R}$, using the flow equations (5.1) for the almost-BPS system. After a lengthy but straightforward computation, we obtain that the derivative of (5.19) is indeed given by

$$
\begin{gathered}
Z(d \hat{R})=\mathrm{T}_{\gamma, Q}^{-} Z(\hat{R})=Z\left(d \mathrm{~T}^{-} \hat{R}\right), \\
Z_{a}(d \hat{R})=\mathrm{T}_{\gamma, Q}^{-} Z_{a}(\hat{R})=Z_{a}\left(d \mathrm{~T}^{-} \hat{R}\right),
\end{gathered}
$$

where we indicated that the result is given by the variation of $\hat{R}$ under the T-duality transformation in (2.62), as shown in (2.63). The values for the one-form generators are given by

$$
\begin{aligned}
\gamma_{-} & =\frac{1}{2}\left[d U-\operatorname{Im} Y e^{2 U} \star d \omega-\frac{1}{3} \operatorname{Im}\left(Y e^{-i \alpha} \Omega \cdot d t\right)\right] \\
\bar{Q}_{-a} & =-\frac{1}{2} \bar{Y}\left[c_{a b c} \bar{\Omega}^{b} e_{j}^{c} d t^{j}-e^{i \alpha} e_{a \bar{\jmath}} d \bar{t}^{\bar{\jmath}}+\frac{1}{3} \Omega_{a}\left(e^{i \alpha} \bar{\Omega}^{b} e_{b \bar{\jmath}} d \bar{t}^{\bar{\jmath}}+2 \frac{Y^{2}}{|Y|^{2}} e^{-i \alpha} \Omega_{j} d t^{j}\right)\right] .
\end{aligned}
$$


The expression for $\gamma_{-}$can be rewritten using (5.5) in a form similar to the corresponding T-duality parameter for the composite non-BPS system in (4.18), as

$$
\gamma_{-}=\frac{1}{6} e^{2 U}(\star d \omega-d M)
$$

where we used the definition (2.50) for the function $M$ in terms of the phases $e^{i \alpha}$ and $\mathcal{N}[\Omega]$. In this form, it is manifest that setting the T-duality parameters $\gamma_{-}, Q_{-}^{a}$ to zero, one finds that the angular momentum is given in terms of a harmonic function, $M$, while the second of (5.23) becomes a reality constraint on the scalar flow, similar to (2.59) in the composite non-BPS system. This restriction therefore leads to the single centre subclass, which is common to both the composite non-BPS and almost-BPS systems.

Applying the considerations of section 2 on the system at hand, we recall that a generic T-duality leaving $\hat{R}^{*}$ invariant is parametrised by a rank three grade $(-1)$ vector of parameters, which we denote by $\mathcal{K}$, so that (5.20)-(5.21) become

$$
\hat{R}=\exp \left[\mathrm{T}_{\mathcal{K}}^{-}\right] R_{0}, \quad d \mathrm{~T}_{\mathcal{K}}^{-} R_{0}=d \mathcal{K} .
$$

In what follows, we will generally suppress the explicit subscript $\mathcal{K}$ from the T-duality generators for simplicity. One checks that $\mathcal{K}$ is indeed a rank three vector, i.e.

$$
\begin{aligned}
I_{4}(d \hat{R}) & =I_{4}\left(d \mathrm{~T}^{-} \hat{R}\right)=I_{4}(d \mathcal{K})=0 \\
\partial_{\mu} \mathrm{T}^{-} \partial_{\nu} \mathrm{T}^{-} \hat{R} & =-\frac{1}{16} I_{4}\left(\partial_{\mu} \mathrm{T}^{-} \hat{R}, \partial_{\nu} \mathrm{T}^{-} \hat{R}, \hat{R}^{*}\right)=-\frac{1}{16} I_{4}\left(\partial_{\mu} \mathcal{K}, \partial_{\nu} \mathcal{K}, \hat{R}^{*}\right) .
\end{aligned}
$$

These equations are clearly dual to the corresponding equations (4.22) for the composite non-BPS system. Alternatively, the same property follows from the fact that $d \mathrm{~T}^{-} R_{0}$ is of grade $(+1)$ and the rank of such a vector is at most three. Indeed, one can directly verify that the vector $d \mathcal{K}$, as defined in (5.25), satisfies the constraint (2.64) and (2.65), which are equivalent to the real constraint

$$
\frac{1}{2} I_{4}^{\prime}\left(R_{0}, \hat{R}^{*}, \mathcal{K}\right)=\left\langle R_{0}, \hat{R}^{*}\right\rangle \mathcal{K} .
$$

These equations are explicit realisations of the general situation discussed in section 2 .

Finally, we stress the difference between the constant very small vector $R_{0}$ of grade $(+3)$ and the original vector in (3.28), which was also used to define $\hat{R}$ in (5.19). This can seem confusing, especially in view of the fact that we used the constant vector $R_{0}$ to define the constraint in (5.28) and ultimately the grading of the vector space. However, as already explained in the analogous situation for the composite non-BPS system, below (4.22), the grading associated to the T-dualities leaving invariant $\hat{R}^{*}$ is only defined up to the action of the T-dualities themselves. In this respect, one can chose any constant vector $R_{0}$ in this orbit to define the grading. Given this redundancy in the definition, it will be convenient to fix $T^{-}$to vanish in the asymptotic region, such that $R_{0}=\left.\hat{R}\right|_{r \rightarrow \infty}$.

\subsection{The linear system}

We are now in a position to use the above results to write the system of flow equations in the real basis, in terms of the symplectic section, $\mathcal{V}$, the two very small constant vectors $\hat{R}^{*}, R_{0}$, and the relevant T-duality generators. 
To show this, we insert (5.16) and (5.17) in (5.11) to eliminate the spurious quantities $W$ and $\mu$ in favor of $V$ and $d V$ respectively. Moreover, it is useful to note the relation

$$
d \mathrm{~T}^{-}\left[2 e^{-U} \operatorname{Im}\left(e^{-i \alpha} \mathcal{V}\right)\right]=2 e^{-U} \operatorname{Im}\left[-3 i \gamma_{*} e^{-i \alpha} \mathcal{V}+i \gamma_{*} \bar{\Omega}^{i} D_{i} \mathcal{V}+Q_{*}^{i} D_{i} \mathcal{V}\right]
$$

as well as the identity

$$
\left(d-2 d \mathrm{~T}^{-}\right) V \hat{R}=d V \hat{R}-V d \hat{R},
$$

which follows from (5.21), and we remind the reader that the explicit expression for $d \hat{R}$ is given by (5.22) with parameters as in (5.23). One can then verify that the expression

$$
\star d w=-\left(d-2 d \mathrm{~T}^{-}\right)\left[2 \operatorname{Im}\left(e^{-U-i \alpha} \mathcal{V}\right)-\frac{1}{2} V \hat{R}-\frac{M}{V} \hat{R}^{*}\right]-\beta \star d \omega \hat{R}^{*},
$$

is equivalent to (5.11) above. This result is manifestly duality covariant in the real basis, as it is written in terms of real symplectic vectors only. In particular, note that (5.31) is completely analogous to the corresponding result (4.30) for the composite non-BPS system, up to the term explicitly proportional to the angular momentum. However, it is simple to shown that this term is unphysical, after considering the electromagnetic potentials as well. These are computed by combining the result (5.8) with (5.16) for the function $W$, to find

$$
\zeta=-2 e^{U} \operatorname{Re}\left[e^{-i \alpha} \mathcal{V}\right]+\left(\beta-\frac{1}{V}\right) \hat{R}^{*},
$$

which leads to the following expression for the total spatial field strengths

$$
\begin{aligned}
F=\zeta d \omega+d w= & -\left(2 e^{U} \operatorname{Re}\left[e^{-i \alpha} \mathcal{V}\right]+\frac{1}{V} \hat{R}^{*}\right) d \omega \\
& -\star\left(d-2 d \mathrm{~T}^{-}\right)\left[2 \operatorname{Im}\left(e^{-U-i \alpha} \mathcal{V}\right)-\frac{1}{2} V \hat{R}-\frac{M}{V} \hat{R}^{*}\right] .
\end{aligned}
$$

Given that the constant $\beta$ does not appear in the gauge invariant total field strengths, we conclude it corresponds to a residual gauge transformation of the type $A \rightarrow A+\beta \hat{R}^{*} d t$ and therefore is unphysical. Henceforth we set $\beta=0$ in all relations, for simplicity. With this choice, (5.31) is formally exactly the same as its counterpart in the composite non-BPS system in (4.30), up to changing the relevant T-dualities from those leaving $R$ invariant to those leaving $R^{*}$ invariant.

Note however that this choice of $\beta$ is not the most convenient one for all purposes, as for example in showing that the above equations describe multi-centre BPS solutions as a particular case. It can be shown that this is the case when $V$ is a constant, but one only recovers the standard form of BPS solutions after imposing $\beta=\frac{1}{V}$, as is clear from equation (5.32).

\subsection{Integration and local structure}

The presence of the T-duality connection $d \mathrm{~T}^{-}$in (5.31) does not allow for a straightforward solution in terms of harmonic functions, but one can follow steps similar to the composite non-BPS system in order to solve the system in terms of local functions. We can write the scalar and vector fields as

$$
2 e^{-U} \operatorname{Im}\left(e^{-i \alpha} \mathcal{V}\right)-\frac{1}{2} V \hat{R}-\frac{M}{V} \hat{R}^{*}=-\mathcal{H}, \quad d w=\star\left(d-2 d \mathrm{~T}^{-}\right) \mathcal{H},
$$


where the vector of functions $\mathcal{H}$ is the solution to the non-harmonic equation

$$
d \star d \mathcal{H}-2 d \star d \mathrm{~T}^{-} \mathcal{H}-2 d \mathrm{~T}^{-} \wedge \star d \mathcal{H}=0 .
$$

This can be simplified and cast as a Poisson equation after introducing a rescaled vector, as

$$
\mathcal{H}_{0}=\exp \left[-\mathrm{T}^{-}\right] \mathcal{H}
$$

which in turn is the solution to the equation

$$
d \star d \mathcal{H}_{0}-d \star d \mathrm{~T}^{-} \mathcal{H}_{0}-d \mathrm{~T}^{-} \wedge \star d \mathrm{~T}^{-} \mathcal{H}_{0}=0 .
$$

This can be formally obtained from (4.37) upon exchange of T-duality transformations. In terms of the new vector, $\mathcal{H}_{0}$, the scalar and vector fields are given by

$$
\begin{aligned}
2 e^{-U} \operatorname{Im}\left(e^{-i \alpha} \mathcal{V}\right) & =-\exp \left[\mathrm{T}^{-}\right]\left(\mathcal{H}_{0}-\frac{1}{2} V R_{0}-\frac{M}{V} \hat{R}^{*}\right), \\
d w & =\exp \left[\mathrm{T}^{-}\right] \star d \mathcal{H}_{0}-\star d\left(\exp \left[\mathrm{T}^{-}\right]\right) \mathcal{H}_{0}
\end{aligned}
$$

which is the final form of the system in the real basis.

The solutions to the above system can be characterised using the fact that the components of the vector $\mathcal{H}_{0}$ are restricted, in the following way. The form (5.38) of the vector fields allows us to compute

$$
\begin{aligned}
Z_{a}\left(\exp \left[\mathrm{T}^{-}\right] \star d \mathcal{H}_{0}\right) & -\mathcal{N}[\bar{\Omega}] \Omega_{a} Z\left(\exp \left[\mathrm{T}^{-}\right] \star d \mathcal{H}_{0}\right) \\
& =Z_{a}\left(\star d w+\exp \left[\mathrm{T}^{-}\right] d \mathrm{~T}^{-} \mathcal{H}_{0}\right)-\mathcal{N}[\bar{\Omega}] \Omega_{a} Z\left(\star d w+\exp \left[\mathrm{T}^{-}\right] d \mathrm{~T}^{-} \mathcal{H}_{0}\right) \\
& =2 \bar{Q}_{-a}-e^{i \alpha} N_{a}+2 i e^{-U} \Omega_{a} \gamma_{-}\left(3 \frac{Y}{|Y|^{2}}-2\right)
\end{aligned}
$$

The crucial observation is that the central charges in (5.39) satisfy the reality constraint (2.59), as

$$
\begin{aligned}
e^{i \alpha} & {\left[\bar{Z}^{a}\left(\exp \left[\mathrm{T}^{-}\right] d \mathcal{H}_{0}\right)-\mathcal{N}[\Omega] \bar{\Omega}^{a} \bar{Z}\left(\exp \left[\mathrm{T}^{-}\right] d \mathcal{H}_{0}\right)\right]=} \\
& =c^{a b c} \Omega_{b} Z_{c}\left(\exp \left[\mathrm{T}^{-}\right] d \mathcal{H}_{0}\right)+\bar{\Omega}^{a}\left(Z\left(\exp \left[\mathrm{T}^{-}\right] d \mathcal{H}_{0}\right)-\mathcal{N}[\Omega] \bar{\Omega}^{b} Z_{b}\left(\exp \left[\mathrm{T}^{-}\right] d \mathcal{H}_{0}\right)\right)
\end{aligned}
$$

which restricts the components of the corresponding vector to lie on a particular Lagrangian subspace [14]. The same constraint holds for the integrated vector $\mathcal{H}_{0}$, for which

$$
Z_{a}\left(\exp \left[\mathrm{T}^{-}\right] \mathcal{H}_{0}\right)-\mathcal{N}[\bar{\Omega}] \Omega_{a} Z\left(\exp \left[\mathrm{T}^{-}\right] \mathcal{H}_{0}\right)=-\Omega_{a} e^{-U} Y .
$$

The real form of this relation is the same as for the composite non-BPS system (4.56), which we recall in this section for completeness

$$
\frac{1}{2} I_{4}^{\prime}\left(R_{0}, \hat{R}^{*}, \mathcal{H}_{0}\right)=-\left\langle R_{0}, \hat{R}^{*}\right\rangle \mathcal{H}_{0}+3\left\langle\mathcal{H}_{0}, \hat{R}^{*}\right\rangle R_{0}, \quad\left\langle R_{0}, \mathcal{H}_{0}\right\rangle=0
$$

where we used (5.25) to undo an overall T-duality on all terms in this equation. The constraints (5.42) are exactly dual to (5.28), as they are related by replacing $\hat{R}^{*}$ and $R_{0}$. 
We therefore conclude that $\mathcal{H}_{0}$ and $\mathcal{K}$ lie in opposite Lagrangian subspaces, i.e. the two vectors have no common directions and span $2 n_{v}+1$ independent components in the $2\left(n_{v}+1\right)$-dimensional vector space.

To be more precise, the vector $\mathcal{K}$ is of grade $(+1)$, as explained in section 5.2 , while the vector $\mathcal{H}_{0}$ and its derivative lie in the Lagrangian subspace composed by grade $(-1)$ and $(+3)$ components in the decomposition (2.29), as

$$
\mathcal{H}_{0} \in\left(\mathbb{R}^{n_{v}}\right)^{(-1)} \oplus \mathbb{R}^{(3)}
$$

exactly as in (4.42). Applying this to (5.37), the following pattern arises for the various terms

$$
\begin{aligned}
& d \star d \mathcal{H}_{0}-d \star d \mathrm{~T}^{-} \mathcal{H}_{0}-d \mathrm{~T}^{-} \wedge \star d \mathrm{~T}^{-} \mathcal{H}_{0}=0, \\
& (-1) \oplus(+3) \quad(-3) \oplus(+1)
\end{aligned}
$$

where we used the fact that $\mathrm{T}^{-}$lowers the grade of a vector by $(-2)$. In direct correspondence with (4.43)-(4.45) for the composite non-BPS system, we find that (5.44) decomposes into two equations according to its graded decomposition, as

$$
\begin{aligned}
d \star d \mathcal{H}_{0}-d \mathrm{~T}^{-} \wedge \star d \mathrm{~T}^{-} \mathcal{H}_{0} & =0, \\
d \star d \mathrm{~T}^{-} & =0,
\end{aligned}
$$

where in the second equation we used the property that no $\mathrm{T}$-duality $\mathrm{T}^{-}$leaves the vector (5.43) invariant. This is true because the grade 3 component of $\mathcal{H}_{0}$ can be identified as the nowhere vanishing harmonic function $V$ defined in (5.15), as

$$
\left\langle\hat{R}^{*}, \mathcal{H}_{0}\right\rangle=-V
$$

as can be seen by contracting (5.38) by $\hat{R}^{*}$. We now analyse each of the two equations (5.45)-(5.46) in turn.

The solution to $(5.46)$ is equivalent to the condition

$$
d \star d \mathcal{K}=0
$$

where we used (5.25). It follows that the vector of parameters $\mathcal{K}$ is a generic grade $(+1)$ vector of harmonic functions, $\mathcal{K}$, which is of rank three. Note that, in this system, the poles of $\mathcal{K}$ represent new independent physical charges, since this vector is by definition linearly independent from $\mathcal{H}_{0}$. In fact, the poles of this function are the only relevant information, since one may always absorb the constant part of $\mathcal{K}$ into $R_{0}$ in (5.25).

We now turn to the Poisson equation (5.45) and observe that the source term is of grade $(-1)$, according to (5.44). It follows that only $n_{v}-1$ out of the $n_{v}$ components of $\mathcal{H}_{0}$ are sourced, leading to an equal number of non-harmonic functions. The remaining component is the harmonic function $V$, already identified in (5.47) above. The source term in (5.45) can be computed explicitly using (2.62), with the result

$$
\left.Z\left(-\exp \left[\mathrm{T}^{-}\right] d \mathrm{~T}^{-} \wedge \star d \mathrm{~T}^{-} \mathcal{H}_{0}\right]\right)=-\frac{i}{64} V \frac{\partial I_{4}}{\partial \bar{Z}}\left(\hat{R}^{*}, d \hat{R}, \star d \hat{R}\right)
$$




$$
Z_{a}\left(-\exp \left[\mathrm{T}^{-}\right] d \mathrm{~T}^{-} \wedge \star d \mathrm{~T}^{-} \mathcal{H}_{0}\right)=\frac{i}{64} V \frac{\partial I_{4}}{\partial \bar{Z}^{a}}\left(\hat{R}^{*}, d \hat{R}, \star d \hat{R}\right) .
$$

i.e. it is proportional to the vector defined in (5.27). This vector is of grade ( -1$)$ by construction and it can be verified to satisfy the constraint in (5.40). We can now rewrite $(5.45)$ as

$$
d \star d \mathcal{H}_{0}=\frac{1}{64} V I_{4}^{\prime}\left(\hat{R}^{*}, d \mathcal{K}, \star d \mathcal{K}\right),
$$

where we used the definition of $\mathcal{K}$ in (5.25) and (5.27).

Finally, we present the covariant form for the equation determining the angular momentum and the function $M$ in (2.50) and (5.24). The starting point is the first of (5.23), which upon use of (5.5) can be written as

$$
\star d \omega-d M=\left\langle\exp \left[\mathrm{T}^{-}\right] \mathcal{H}_{0}, \star d w\right\rangle=\left\langle\mathcal{H}_{0}, d \mathcal{H}_{0}-d \mathrm{~T}^{-} \mathcal{H}_{0}\right\rangle,
$$

where we also used (5.38) in the second equality. Explicit computation of the last expression using the definition (2.36) leads to the alternative form

$$
\star d \omega-d M=-\frac{1}{2} V\left\langle\mathcal{H}_{0}, d \hat{R}\right\rangle=-\frac{1}{2} V\left\langle\mathcal{H}_{0}, d \mathcal{K}\right\rangle .
$$

Taking the divergence and the curl of this equation one obtains the relevant equations for the function $M$ and the angular momentum respectively. The resulting Poisson equation for $M$ reads

$$
d \star d M=\frac{1}{2} d\left(V\left\langle\mathcal{H}_{0}, \star d \mathcal{K}\right\rangle\right),
$$

and can be solved once $\mathcal{H}_{0}$ and $\mathcal{K}$ are specified. Note that upon setting the parameters, $\mathcal{K}$, of the T-dualities to vanish, these equations imply that $M$ is a harmonic function, while $\omega$ is the corresponding dual one-form, consistent with the single centre class.

This concludes our duality covariant presentation of the almost-BPS system in terms of the real basis. In the next section, we summarise the final form of the equations to be solved and we comment on some of the properties of solutions.

\subsection{Summary of results}

In this short section, we summarise the relevant formulae for the almost-BPS system in the real basis. All relations presented here were shown explicitly in the previous sections and we refer to the discussion there for further details. We find it however useful, both for clarity and for future applications, to give a as self-contained as possible account of the final form of the system.

The ansatze for the metric and gauge fields are given in (3.1) and (3.3) in terms of the function $e^{U}$, the one-form $\omega$ and the spatial vector fields $d w$, while the electromagnetic potentials are fixed by (5.32). The first order equation for the almost-BPS system is given by (5.31), as

$$
\star d w=-\left[d-2 d \mathrm{~T}_{\mathcal{K}}^{-}\right]\left[2 \operatorname{Im}\left(e^{-U-i \alpha} \mathcal{V}\right)-\frac{1}{2} V \hat{R}-\frac{M}{V} \hat{R}^{*}\right] .
$$


Here, $M, V$, are functions to be specified below, while $\hat{R}^{*}$ and $\hat{R}$ are a constant and a non-constant very small vector respectively, where $\left\langle\hat{R}, \hat{R}^{*}\right\rangle=4$. Here, the non-constant $\hat{R}$ is related to a constant very small vector, $R_{0}^{*}$, by $(5.20)$

$$
\hat{R}=\exp \left[\mathrm{T}_{\mathcal{K}}^{-}\right] R_{0}
$$

which again satisfies $\left\langle R_{0}, \hat{R}^{*}\right\rangle=4$. In all equations, $\mathrm{T}_{\mathcal{K}}^{-}$is a generator of the $\mathrm{T}$-dualities leaving $\hat{R}^{*}$ invariant, parametrised by a vector of harmonic functions, $\mathcal{K}$. As discussed in section 2 , the vector parameter $\mathcal{K}$ lies in the grade $(+1)$ component of the vector space according to the decomposition implied by the T-duality. It is therefore a three-charge vector satisfying

$$
\frac{1}{2} I^{\prime}\left(R_{0}, \hat{R}^{*}, \mathcal{K}\right)=\left\langle R_{0}, \hat{R}^{*}\right\rangle \mathcal{K}
$$

which indeed specifies a vector of $n_{v}$ degrees of freedom.

The solutions to the flow equation (5.54) are simplified by introducing a vector, $\mathcal{H}_{0}$, of grade $(-1) \oplus(+3)$, i.e. satisfying

$$
\frac{1}{2} I_{4}^{\prime}\left(R_{0}, \hat{R}^{*}, \mathcal{H}_{0}\right)=-\left\langle R_{0}, \hat{R}^{*}\right\rangle \mathcal{H}_{0}+3\left\langle\mathcal{H}_{0}, \hat{R}^{*}\right\rangle R_{0} .
$$

Note that (5.56) follows from a similar constraint, obtained by interchanging $\hat{R}^{*}$ with $R_{0}$, that projects to the $(+1) \oplus(-3)$ component of the vector space. The equations resulting from (5.54) upon use of $\mathcal{H}_{0}$ in (5.57), take the form (5.38),

$$
\begin{aligned}
2 e^{-U} \operatorname{Im}\left(e^{-i \alpha} \mathcal{V}\right) & =-\exp \left[\mathrm{T}_{\mathcal{K}}^{-}\right]\left(\mathcal{H}_{0}-\frac{1}{2} V R_{0}-\frac{M}{V} \hat{R}^{*}\right), \\
\star d w & =\exp \left[\mathrm{T}_{\mathcal{K}}^{-}\right]\left(d \mathcal{H}_{0}-d \mathrm{~T}_{\mathcal{K}}^{-} \mathcal{H}_{0}\right)
\end{aligned}
$$

where $V$ is now identified with the grade $(+3)$ component of $\mathcal{H}_{0}$, as $V=\left\langle\mathcal{H}_{0}, \hat{R}^{*}\right\rangle$. The compatibility relation for these relations leads to the field equation for $\mathcal{H}_{0}$, as in (5.50)

$$
d \star d \mathcal{H}_{0}=d \mathrm{~T}_{\mathcal{K}}^{-} \wedge \star d \mathrm{~T}_{\mathcal{K}}^{-}=\frac{1}{64} V I_{4}\left(d \mathcal{K}, \star d \mathcal{K}, \hat{R}^{*}\right) .
$$

As the right hand side of this relation is a vector of grade $(-1)$, the corresponding components of $\mathcal{H}_{0}$ are not harmonic, whereas $V$ is, as can be seen by taking the inner product of $(5.60)$ with $\hat{R}^{*}$

$$
\left\langle\hat{R}^{*}, d \star d \mathcal{H}_{0}\right\rangle=d \star d V=0,
$$

where we used (5.56). The final dynamical equation required is the one for the function $M$ in (5.58) and the angular momentum vector $\omega$, both of which are conveniently given by $(5.52)$, as

$$
\star d \omega-d M=\left\langle\mathcal{H}_{0}, d \mathcal{H}_{0}-d \mathrm{~T}_{\mathcal{K}}^{-} \mathcal{H}_{0}\right\rangle=-\frac{1}{2} V\left\langle\mathcal{H}_{0}, d \mathcal{K}\right\rangle .
$$

Taking the divergence of this equation, one obtains a Poisson equation for $M$.

The equations above can be seen to be equivalent to the known formulation of the almost-BPS system, as given in five dimensional supergravity [15-17], by making a choice for the constant vectors $R_{0}$ and $\hat{R}^{*}$. Indeed, upon choosing

$$
R_{0} \propto\left(0, \delta_{I}^{0}\right), \quad \hat{R}^{*} \propto\left(\delta_{0}^{I}, 0\right),
$$


where we disregard the (arbitrary) normalisation, one can show a complete equivalence of the above to the original system in [15]. This particular frame is convenient in that it allows to lift to five dimensional solutions that are locally but not globally supersymmetric. However, our formulation of the almost-BPS system is closed under four dimensional dualities and includes all duals of the system in [15]. More recently, it was shown in [30] that some of the BPS structure is preserved in four dimensions as well, upon reinterpreting the constant vector $\hat{R}^{*}$ as Fayet-Iliopoulos terms in a gauged theory.

We close with some comments on the structure of the solutions. First, the physical scalars and the metric scale factor can be obtained by solving (5.58) in the standard way [8], once $\mathcal{H}_{0}$ and $M$ are solved for. Since all quantities above are appropriate combinations of the single centre solution in [14], up to overall T-dualities, it is possible to use many of the results given there. For instance, the metric scale factor is given by (4.65), as

$$
e^{-4 U}=-I_{4}\left(\mathcal{H}_{0}\right)-M^{2},
$$

in exactly the same way as for the composite non-BPS system. However, in this case the situation is richer and more complicated, in view of the fact that the grade $(-1)$ components of $\mathcal{H}_{0}$ are not harmonic and its grade 3 component $V$ does not necessarily carry a pole at all centres. Indeed, it turns out that not all black holes described by the almost-BPS system are non-supersymmetric in isolation. On the contrary, the presence of both BPS and non-BPS types of centres, is the distinguishing property of this system, as shown in [15-17]. Clearly, the fact that the harmonic functions $\mathcal{K}$ lie in a subspace independent of the one where $\mathcal{H}_{0}$ lives is the crucial ingredient that allows for both BPS and non-BPS types of charges to exist simultaneously.

As seen in the case of the composite non-BPS system, solutions do not exist for all charge configurations and this holds also in the almost-BPS system. Moreover, it is not possible to obtain arbitrary asymptotic moduli for a given allowed charge configuration, for exactly the same reasons explained in section 4.5. Indeed, (4.53) and (4.58) have exactly the same structure in both cases, so that some of the $n_{v}+1$ asymptotic constants in $\mathcal{H}_{0}$ and the parameters of $\hat{R}$ and $R_{0}^{*}$ will correspond to charges rather than moduli. We once again refer to [27] for more details on the structure of almost-BPS solutions in four dimensions and for explicit examples.

\section{Conclusion}

In this paper, we gave a comprehensive treatment of the flow equations describing multi-centre under-rotating black holes in $\mathcal{N}=2, D=4$ supergravity coupled to vector multiplets with a symmetric scalar manifold. In particular, we considered the non-linear sigma model obtained after timelike dimensional reduction to three dimensions and derived the general, frame independent, flow equations for two systems of multi-centre non-BPS black holes, namely the composite non-BPS and almost-BPS systems.

This represents a generalisation of the systems given in specific frames in [12, 15], to systems that are closed under electric/magnetic duality. The resulting structure for the vector fields and scalars in terms of real symplectic vectors turns out to be very similar 
for both systems. In particular, both systems are described in terms of space-dependent transformations along abelian subgroups of isometries on the scalar target space. In terms of the natural embedding to string theories, these subgroups of the full duality group are conjugate to the so-called spectral flow transformations, that are combinations of T-dualities with gauge transformations on the $p$-form gauge fields. In this paper, we refer to them simply as T-dualities for brevity.

The main distinction between these solutions and the BPS multi-centre solutions, is that the electromagnetic vector fields are not harmonic anymore, but satisfy instead

$$
d \star d w-2 d \mathrm{~T}_{\mathcal{K}}^{ \pm} \wedge \star d w=0,
$$

where the functions $\mathcal{K}$ are themselves harmonic. Note that the consistency of this equation requires that the generators $\mathrm{T}^{ \pm}$are indeed abelian, as for $\mathrm{T}$-dualities. It follows from this equation that the poles of the harmonic functions $\mathcal{K}$ contribute to the electromagnetic charges in a non-linear way. Despite the interpretation of these functions as parameters of abelian isometries of the scalar manifold, they are not associated to a gauging of the theory.

The crucial property that makes a general discussion in terms of covariant objects possible is that the action of general T-dualities can be given explicitly using the quartic invariant, $I_{4}$, of symmetric special Kähler geometry. Indeed, as summarised in sections 4.5 and 5.5, all relevant equations are written in terms of this invariant only, evaluated for the real vectors parametrising the solutions. In this form, these duality covariant systems are not significantly more complicated than the equations given for the composite non-BPS system in [12] and for the almost-BPS system in [15] and can be solved in exactly the same way.

The main advantage of the formulation displayed in this paper is that one need not define solutions in a fixed duality frame in terms of generic parameters, and only compute the electromagnetic charges and asymptotic moduli a posteriori, as in the constructions of $[12,13,16,17]$. In contrast, one can start from any configuration of physical charges satisfying the required criteria associated to each system and construct the corresponding solution, using the results summarised in sections 4.5 and 4.5 . This is in particular very useful for studying the domain of stability of these solutions in moduli space. Using this formulation, one can start from a given set of electromagnetic charges consistent with the system (e.g. they have to all mutually commute with a common very small vector $R$ in the composite non-BPS system), and parametrize the most general very small vectors $R$ and $R^{*}$ satisfying the corresponding constraints. Using the formulation of this paper, one can then determine the most general solution associated to a given charge configuration and define the domain of existence of such solutions in moduli space. As opposed to BPS solutions, the domain of existence of such solutions in moduli space will be restricted to a hypersurface of non-zero co-dimension. The normal directions to the hypersurface are probably not forbidden physically, but rather push us out of the domain where we know how to describe the solution. For example, the BPS solutions within the almost-BPS system only exist on a co-dimension one hypersurface, but only due to the fact that the charges at all centres must be compatible with a single constant vector $R_{0}^{*}$, leading to a subset of all BPS solutions. Nonetheless, one can still wonder if there are walls of marginal stability within the hypersurfaces defined by each of the two systems described in this paper, i.e. 
whether there are boundaries of the domain of existence of such solutions at finite values of the moduli. We intend to study two-centre configurations in the aim of exhibiting (or not) walls of marginality for non-BPS solutions in a forthcoming publication.

From a more general point of view, the unified description of the two known non-BPS systems and its relative simplicity are encouraging for further uncovering the structure of non-BPS solutions in supergravity. In particular, the isometries of the scalar manifold seem to play a crucial role not only in the effective three-dimensional theory, but also in the real formulation in four dimensions. It would be interesting to understand the role of these isometries in the reduction of the equations of motion to first order systems, which is not clear from our treatment in terms of nilpotent orbits. Indeed, while the two systems presented in this paper exhaust the possibilities for simple models (e.g. the STU model [12]), this is not the case for more complicated models, admitting higher nilpotent orbits. The latter define generalisations of the known systems for which one trades the central charge for the largest eigen value of its Kähler derivative, understood as a Jordan algebra element. These more complicated systems of non-BPS solutions satisfy more involved solvable systems of differential equations (see [13] for example in the context of maximal supergravity) and one might hope that structures similar to the ones described in this paper appear in those cases as well.

\section{Acknowledgments}

We thank Hermann Nicolai for pointing out to us reference [31]. This work was supported by the French ANR contract 05-BLAN-NT09-573739, the ERC Advanced Grant no. 226371 and the ITN programme PITN-GA-2009-237920. The work of SK was supported in part by the ANR grant 08-JCJC-0001-0, and by the ERC Starting Independent Researcher Grant 240210-String-QCD-BH.

\section{A $\mathcal{N}=2$ supergravity and symmetric special Kähler geometry}

The bosonic Lagrangian of $\mathcal{N}=2$ supergravity coupled to $n_{v}$ vector multiplets reads $[36,37]$

$$
8 \pi e^{-1} \mathcal{L}=-\frac{1}{2} R-i\left\langle D^{\mu} \overline{\mathcal{V}}, D_{\mu} \mathcal{V}\right\rangle-\frac{1}{4} F_{\mu \nu}^{I} G_{I}^{\mu \nu}
$$

Here, the $F_{\mu \nu}^{I}=\partial_{\mu} A_{\nu}^{I}-\partial_{\nu} A_{\mu}^{I}$ for $I=0, \ldots n_{v}$ encompass the graviphoton and the gauge fields of the vector multiplets and $G_{I}^{\mu \nu}$ are the dual field strengths, defined in terms of the $F_{\mu \nu}^{I}$ though the scalar dependent couplings, whose explicit form will not be relevant in what follows. The gauge field equations of motion and Bianchi identities can then be cast as a Bianchi identity on the symplectic vector

$$
\mathcal{F}_{\mu \nu}=\left(\begin{array}{c}
F_{\mu \nu}^{I} \\
G_{I \mu \nu}
\end{array}\right),
$$

whose integral over any two-cycle defines the associated electromagnetic charges through

$$
\Gamma=\left(\begin{array}{l}
p^{I} \\
q_{I}
\end{array}\right)=\frac{1}{2 \pi} \int_{S^{2}} \mathcal{F} .
$$


The physical scalar fields $t^{i}$, which parametrize a special Kähler space $\mathcal{M}_{4}$ of complex dimension $n_{v}$, only appear in (A.1) through the section, $\mathcal{V}$, of a holomorphic $\mathrm{U}(1) \times \operatorname{Sp}\left(2 n_{v}+2, \mathbb{R}\right)$ bundle over $\mathcal{M}_{4}$. Choosing a basis, this section can be written in components in terms of scalars $X^{I}$ as

$$
\mathcal{V}=\left(\begin{array}{c}
X^{I} \\
F_{I}
\end{array}\right), \quad F_{I}=\frac{\partial F}{\partial X^{I}},
$$

where $F$ is a holomorphic function of degree two, called the prepotential, which we will always consider to be cubic

$$
F=-\frac{1}{6} c_{i j k} \frac{X^{i} X^{j} X^{k}}{X^{0}} \equiv-\frac{\mathcal{N}[X]}{X^{0}}
$$

for completely symmetric $c_{i j k}, i=1, \ldots n_{v}$, and we introduced the cubic norm $\mathcal{N}[X]$. The section $\mathcal{V}$ is subject to the constraint

$$
\langle\overline{\mathcal{V}}, \mathcal{V}\rangle=i
$$

and is uniquely determined by the physical scalar fields $t^{i}=\frac{X^{i}}{X^{0}}$ up to a local U(1) transformation. The $\mathrm{U}(1)$ gauge invariance of (A.1) is ensured by the appearance of the Kähler connection $Q_{\mu}$ in the covariant derivative. The Kähler potential on $\mathcal{M}_{4}$ is defined up to an arbitrary holomorphic function $f(t)$ as

$$
\mathcal{K}=-\ln (i \mathcal{N}[t-\bar{t}])+f(t)+f(\bar{t})
$$

and we fixed the $\mathrm{U}(1)$ gauge invariance in terms of Kähler transformations by requiring that the Kähler connection is determined by the Kähler potential as

$$
Q=\operatorname{Im}\left[\partial_{i} \mathcal{K} d t^{i}\right]
$$

such that

$$
g_{i \bar{\jmath}}=\partial_{i} \partial_{\bar{\jmath}} \mathcal{K}, \quad D_{\mu} \mathcal{V}=\left(\partial_{\mu}+i Q_{\mu}\right) \mathcal{V}=D_{i} \mathcal{V} \partial_{\mu} t^{i}=\left(\partial_{i} \mathcal{V}+\frac{1}{2} \partial_{i} \mathcal{K} \mathcal{V}\right) \partial_{\mu} t^{i}
$$

where $D_{i} \mathcal{V}$ is the corresponding Kähler covariant derivative on the components of the section. With the prepotential (A.5), the special geometry identities [38] reduce to

$$
\bar{D}_{\bar{\jmath}} D_{i} \mathcal{V}=g_{i \bar{\jmath}} \mathcal{V}, \quad D_{i} D_{j} \mathcal{V}=i e^{\mathcal{K}} c_{i j k} g^{k \bar{k}} \bar{D}_{\bar{k}} \overline{\mathcal{V}},
$$

which are used throughout the main text.

We introduce the following notation for any symplectic vector $J$

$$
Z(J)=\langle J, \mathcal{V}\rangle, \quad Z_{i}(J)=\left\langle J, D_{i} \mathcal{V}\right\rangle,
$$

with the understanding that when the argument is form valued, the operation is applied component wise. For instance, the central charge of the gauge field is

$$
Z(\mathcal{F})=e^{\frac{\mathcal{K}}{2}}\left(G_{0}+t^{i} G_{i}+\frac{1}{2} c_{i j k} t^{i} t^{j} F^{k}-\mathcal{N}[t] F^{0}\right)
$$


for the prepotential (A.5). With these definitions it is possible to introduce a scalar dependent complex basis for symplectic vectors, given by $\left(\mathcal{V}, D_{i} \mathcal{V}\right)$, so that any vector $J$ can be expanded as

$$
J=2 \operatorname{Im}\left[-\bar{Z}(J) \mathcal{V}+g^{\bar{\imath} j} \bar{D}_{\bar{\imath}} \bar{Z}(J) D_{j} \mathcal{V}\right],
$$

whereas the symplectic inner product can be expressed as

$$
\left\langle J_{1}, J_{2}\right\rangle=2 \operatorname{Im}\left[-Z\left(J_{1}\right) \bar{Z}\left(J_{2}\right)+Z_{a}\left(J_{1}\right) \bar{Z}^{a}\left(J_{2}\right)\right] .
$$

Finally, we introduce the notion of complex selfduality of the gauge fields (A.2), which satisfy the identity

$$
\mathrm{J} \mathcal{F}=-* \mathcal{F},
$$

where $\mathrm{J}$ is a scalar dependent complex structure defined as

$$
\mathrm{J} \mathcal{V}=-i \mathcal{V}, \quad \mathrm{J} D_{i} \mathcal{V}=i D_{i} \mathcal{V}
$$

Open Access. This article is distributed under the terms of the Creative Commons Attribution License which permits any use, distribution and reproduction in any medium, provided the original author(s) and source are credited.

\section{References}

[1] S. Ferrara, R. Kallosh and A. Strominger, $\mathcal{N}=2$ extremal black holes, Phys. Rev. D 52 (1995) 5412 [hep-th/9508072] [INSPIRE].

[2] S. Ferrara and R. Kallosh, Supersymmetry and attractors, Phys. Rev. D 54 (1996) 1514 [hep-th/9602136] [INSPIRE].

[3] A. Strominger, Macroscopic entropy of $\mathcal{N}=2$ extremal black holes, Phys. Lett. B 383 (1996) 39 [hep-th/9602111] [INSPIRE].

[4] A. Strominger and C. Vafa, Microscopic origin of the Bekenstein-Hawking entropy, Phys. Lett. B 379 (1996) 99 [hep-th/9601029] [INSPIRE].

[5] J.M. Maldacena, A. Strominger and E. Witten, Black hole entropy in M-theory, JHEP 12 (1997) 002 [hep-th/9711053] [INSPIRE].

[6] F. Denef, Supergravity flows and D-brane stability, JHEP 08 (2000) 050 [hep-th/0005049] [INSPIRE].

[7] K. Behrndt, D. Lüst and W.A. Sabra, Stationary solutions of $\mathcal{N}=2$ supergravity, Nucl. Phys. B 510 (1998) 264 [hep-th/9705169] [INSPIRE].

[8] B. Bates and F. Denef, Exact solutions for supersymmetric stationary black hole composites, JHEP 11 (2011) 127 [hep-th/0304094] [INSPIRE].

[9] G. Lopes Cardoso, B. de Wit, J. Kappeli and T. Mohaupt, Stationary BPS solutions in $\mathcal{N}=2$ supergravity with $R^{2}$ interactions, JHEP 12 (2000) 019 [hep-th/0009234] [INSPIRE].

[10] A. Dabholkar, F. Denef, G.W. Moore and B. Pioline, Precision counting of small black holes, JHEP 10 (2005) 096 [hep-th/0507014] [INSPIRE].

[11] F. Denef and G.W. Moore, Split states, entropy enigmas, holes and halos, JHEP 11 (2011) 129 [hep-th/0702146] [INSPIRE]. 
[12] G. Bossard and C. Ruef, Interacting non-BPS black holes, Gen. Rel. Grav. 44 (2012) 21 [arXiv: 1106.5806] [INSPIRE].

[13] G. Bossard, Octonionic black holes, JHEP 05 (2012) 113 [arXiv: 1203.0530] [InSPIRE].

[14] G. Bossard and S. Katmadas, Duality covariant non-BPS first order systems, JHEP 09 (2012) 100 [arXiv:1205.5461] [INSPIRE].

[15] K. Goldstein and S. Katmadas, Almost BPS black holes, JHEP 05 (2009) 058 [arXiv: 0812.4183] [INSPIRE].

[16] I. Bena, G. Dall'Agata, S. Giusto, C. Ruef and N.P. Warner, Non-BPS black rings and black holes in Taub-NUT, JHEP 06 (2009) 015 [arXiv:0902.4526] [INSPIRE].

[17] I. Bena, S. Giusto, C. Ruef and N.P. Warner, Multi-center non-BPS black holes: the solution, JHEP 11 (2009) 032 [arXiv:0908.2121] [INSPIRE].

[18] R. Emparan and G.T. Horowitz, Microstates of a neutral black hole in M-theory, Phys. Rev. Lett. 97 (2006) 141601 [hep-th/0607023] [INSPIRE].

[19] A. Dabholkar, A. Sen and S.P. Trivedi, Black hole microstates and attractor without supersymmetry, JHEP 01 (2007) 096 [hep-th/0611143] [INSPIRE].

[20] I. Bena, N. Bobev and N.P. Warner, Spectral flow and the spectrum of multi-center solutions, Phys. Rev. D 77 (2008) 125025 [arXiv: 0803.1203] [INSPIRE].

[21] G. Dall'Agata, S. Giusto and C. Ruef, U-duality and non-BPS solutions, JHEP 02 (2011) 074 [arXiv: 1012.4803] [INSPIRE].

[22] S. Ferrara, A. Marrani, A. Shcherbakov and A. Yeranyan, Multi-centered first order formalism, JHEP 05 (2013) 127 [arXiv:1211.3262] [INSPIRE].

[23] P. Galli, K. Goldstein, S. Katmadas and J. Perz, First-order flows and stabilisation equations for non-BPS extremal black holes, JHEP 06 (2011) 070 [arXiv: 1012.4020] [INSPIRE].

[24] M. Cvetič and D. Youm, All the static spherically symmetric black holes of heterotic string on a six torus, Nucl. Phys. B 472 (1996) 249 [hep-th/9512127] [INSPIRE].

[25] T. Ortín, Extremality versus supersymmetry in stringy black holes, Phys. Lett. B 422 (1998) 93 [hep-th/9612142] [INSPIRE].

[26] R. Kallosh, N. Sivanandam and M. Soroush, Exact attractive non-BPS STU black holes, Phys. Rev. D 74 (2006) 065008 [hep-th/0606263] [INSPIRE].

[27] G. Bossard and S. Katmadas, Multi-centre black hole solutions in four dimensions, in preparation.

[28] B. de Wit and A. Van Proeyen, Isometries of special manifolds, hep-th/9505097 [INSPIRE].

[29] M. Günaydin, G. Sierra and P. Townsend, The geometry of $\mathcal{N}=2$ Maxwell-Einstein supergravity and Jordan algebras, Nucl. Phys. B 242 (1984) 244 [INSPIRE].

[30] K. Hristov, S. Katmadas and V. Pozzoli, Ungauging black holes and hidden supercharges, JHEP 01 (2013) 110 [arXiv:1211.0035] [INSPIRE].

[31] J.R. Faulkner, A construction of Lie algebras from a class of ternary algebras, Trans. Amer. Math. Soc. 155 (1971) 397.

[32] S. Ferrara and M. Günaydin, Orbits of exceptional groups, duality and BPS states in string theory, Int. J. Mod. Phys. A 13 (1998) 2075 [hep-th/9708025] [INSPIRE]. 
[33] P. Breitenlohner, D. Maison and G.W. Gibbons, Four-dimensional black holes from Kaluza-Klein theories, Commun. Math. Phys. 120 (1988) 295 [INSPIRE].

[34] G. Bossard, 1/8 BPS black hole composites, arXiv:1001.3157 [INSPIRE].

[35] S. Ferrara and S. Sabharwal, Quaternionic manifolds for type II superstring vacua of Calabi-Yau spaces, Nucl. Phys. B 332 (1990) 317 [InSPIRE].

[36] B. de Wit and A. Van Proeyen, Potentials and symmetries of general gauged $\mathcal{N}=2$ supergravity: Yang-Mills models, Nucl. Phys. B 245 (1984) 89 [INSPIRE].

[37] B. de Wit, P. Lauwers and A. Van Proeyen, Lagrangians of $\mathcal{N}=2$ supergravity-matter systems, Nucl. Phys. B 255 (1985) 569 [INSPIRE].

[38] A. Ceresole, R. D'Auria and S. Ferrara, The symplectic structure of $\mathcal{N}=2$ supergravity and its central extension, Nucl. Phys. Proc. Suppl. 46 (1996) 67 [hep-th/9509160] [InSPIRE]. 